This is a post-print of an article published in ICARUS (doi: 10.1016/j.icarus.2021.114687).

\title{
Stress and Strain during Shock Metamorphism
}

\author{
Auriol S. P. Rae ${ }^{1,2^{*}}$, Michael H. Poelchau ${ }^{1}$, and Thomas Kenkmann ${ }^{1}$ \\ ${ }^{1}$ Institute of Earth and Environmental Sciences - Geology, Albert-Ludwigs Universität \\ Freiburg, Albertstraße 23b, 79104 Freiburg, Germany. \\ ${ }^{2}$ Department of Earth Sciences, University of Cambridge, Cambridge CB2 3EQ, UK. \\ *Corresponding author email: auriol.rae@geologie.uni-freiburg.de
}

\begin{abstract}
Shock metamorphism is the process by which rocks, and the minerals within them, transform during the passage of a shock wave. Shock effects, such as shatter cones, are critical for the identification of impact structures and have commonly been used to infer mechanical and structural information on the cratering process. To make these inferences, the mechanics of shock wave behavior must be understood. Here, we use numerical simulations to demonstrate how stresses act during shock metamorphism across all regions of the target that experience solid-state shock metamorphism. Furthermore, our numerical simulations predict the strains that are produced as a consequence of those stresses. The results show that the magnitude and orientation of stress and strain during shock metamorphism are variable throughout the target, both spatially and temporally, even between rocks that experience the same peak shock pressure. The provenance of a sample relative to the point of impact and the timing/mechanism of the formation of a shock effect must both be considered when making structural interpretations. The stress and strain magnitudes and orientations as functions of time and location presented in this study provide the constraints that enable a greater understanding of the formation of a variety of shock deformation effects. We demonstrate this with a case study of shatter cones at the Gosses Bluff impact structure. Our results provide a useful guide to assist geologists and petrologists in the interpretation of shock metamorphic effects.
\end{abstract}


This is a post-print of an article published in ICARUS (doi: 10.1016/j.icarus.2021.114687).

\section{Keywords}

Impact Cratering; Shock Metamorphism; Stress; Strain; Deformation; Shatter Cones

Highlights

- We show the orientation and magnitude of stress and strain in shock waves during impact cratering.

- Stress and strain rotation during natural shock events is a fundamental geometrical consequence of the interaction of the shock and rarefaction waves.

- Structural interpretations of impact craters using simplified assumptions of a radial orientation of shock metamorphic effects can now be improved.

- Understanding stress and strain orientation during shock can constrain the mechanism and timing of specific shock deformation effects, such as shatter cones. 
This is a post-print of an article published in ICARUS (doi: 10.1016/j.icarus.2021.114687).

\section{Symbols}

\begin{tabular}{|c|c|}
\hline $\mathbf{L}$ & Impactor diameter \\
\hline $\mathbf{V} \mathbf{i}$ & Impactor velocity \\
\hline $\boldsymbol{\rho}$ & Target density \\
\hline $\boldsymbol{\delta}$ & Impactor density \\
\hline d & Equivalent depth of burst \\
\hline HEL & Hugoniot elastic limit \\
\hline $\mathbf{P}$ & Pressure, the average principal stress \\
\hline Phel & Pressure at the Hugoniot elastic limit \\
\hline $\mathbf{P}_{\max }$ & Maximum pressure \\
\hline$\sigma_{1}>\sigma_{2}>\sigma_{3}$ & Principal stresses, positive in compression \\
\hline $\mathbf{S}_{1}>\mathbf{s}_{2}>\mathbf{s}_{3}$ & $\begin{array}{l}\text { Deviatoric principal stresses, principal stresses relative to the } \\
\text { pressure, } \mathrm{s}_{1,2,3}=\sigma_{1,2,3}-\mathrm{P} \text {. }\end{array}$ \\
\hline $\mathbf{e}_{1}>\mathbf{e}_{2}>\mathbf{e}_{3}$ & Principal strain, positive in extension \\
\hline $\mathbf{S}_{\mathbf{1}}>\mathbf{S}_{\mathbf{2}}>\mathbf{S}_{\mathbf{3}}$ & Principal stretches, $\mathrm{S}_{1,2,3}=1+\mathrm{e}_{1,2,3}$ \\
\hline$\dot{\boldsymbol{S}}_{1}>\dot{\boldsymbol{S}}_{2}>\dot{\boldsymbol{S}}_{3}$ & Principal rates of stretching \\
\hline ISA & Instantaneous stretching axes \\
\hline $\mathbf{W}_{\mathbf{k}}$ & Kinematic vorticity number \\
\hline $\mathbf{k}$ & Instantaneous Flinn parameter, $k=\ln \left(\frac{\dot{S}_{1}}{\dot{S}_{2}}\right) / \ln \left(\frac{\dot{S}_{2}}{\dot{S}_{3}}\right)$ \\
\hline $\mathbf{r}$ & Radial distance from the estimated point source \\
\hline$\alpha$ & $\begin{array}{l}\text { Plunge of radial transect, as measured from the estimated point } \\
\text { source between the horizontal and the line of transect }\end{array}$ \\
\hline$\theta_{\mathrm{PP}}$ & $\begin{array}{l}\text { The angular deviation between a principal stress or instantaneous } \\
\text { stretching axis and the radial orientation relative to the equivalent } \\
\text { depth of burst at the time of peak pressure }\end{array}$ \\
\hline$\theta_{\mathbf{R}}$ & $\begin{array}{l}\text { The angular deviation between a principal stress or instantaneous } \\
\text { stretching axis and the radial orientation relative to the equivalent } \\
\text { depth of burst at the time of shock release }\end{array}$ \\
\hline$\theta_{\text {rise }}$ & $\begin{array}{l}\text { The angular deviation between a principal finite strain orientation } \\
\text { and the radial orientation relative to the equivalent depth of burst } \\
\text { during the rise phase }\end{array}$ \\
\hline$\theta_{\text {rel }}$ & $\begin{array}{l}\text { The angular deviation between a principal finite strain orientation } \\
\text { and the radial orientation relative to the equivalent depth of burst } \\
\text { during the release phase }\end{array}$ \\
\hline$\Delta \theta$ & The total rotation of a principal direction between specified times \\
\hline
\end{tabular}


This is a post-print of an article published in ICARUS (doi: 10.1016/j.icarus.2021.114687).

\section{Introduction}

Hypervelocity impact, where the impact velocity is greater than the speed of sound in the target and/or impactor material, results in the formation of a shock wave (Melosh, 1989). Shock waves cause irreversible deformation, heating, and acceleration of the material through which they pass (Melosh, 1989). The permanent deformation caused by shock waves is known as shock metamorphism. The effects of shock metamorphism include: fracturing, brecciation, mineral kinking, deformation bands, mineral twinning, dislocation glide, solidstate amorphization, solid-state phase transformations, melting, and vaporization (Stöffler and Langenhorst, 1994; French, 1998; French and Koeberl, 2010; Stöffler et al., 2018). Some, but not all, of these shock metamorphic effects are unique to shock metamorphism (French and Koeberl, 2010).

The formation of specific shock metamorphic effects, also known as shock deformation effects, is primarily controlled by the pressure of the shock wave, i.e. the average stress, a hydrostatic scalar value. However, for as long as shock metamorphic effects have been identified, geologists have been interested in the potential for them to provide information on deviatoric stresses and strains during shock wave passage and more particularly relating deformation to the orientation of the shock wave. Examples of shock deformation effects that have been linked to the orientation of a shock wave include: shatter cones (Dietz, 1947, 1967; Dietz and Butler, 1964; Gash, 1971; Milton, 1977; Roddy and Davis, 1977; Milton et al., 1996a), feather features (FFs) (Poelchau and Kenkmann, 2011; Ebert et al., 2020), mineral twinning (Schedl, 2006; Timms et al., 2012, 2017a, 2017b, 2019; Erickson et al., 2016; Cox et al., 2018; Kovaleva and Habler, 2019; Kovaleva et al., 2020), solid-state phase transformations (Leroux et al., 1999; Cavosie et al., 2015; Erickson et al., 2017; Timms et al., 2017a), planar fractures (PFs) (Trepmann, 2008; Agarwal et al., 2016; Rae et al., 2019b; Pittarello et al., 2020), mineral kinking (Hörz and Ahrens, 1969; Graup, 
This is a post-print of an article published in ICARUS (doi: 10.1016/j.icarus.2021.114687).

1978; Dressler, 1990; Agarwal et al., 2019; Ebert et al., 2021), basal planar deformation features (PDFs) in quartz (McLaren et al., 1967; Trepmann and Spray, 2006; Trepmann, 2008; Ebert et al., 2021), PDFs in quartz and feldspar generally (Goltrant et al., 1991, 1992; Pittarello et al., 2020), and strain markers such as flattened chondrules in meteorites (Nakamura et al., 1995, 2000).

\section{The Current Paradigm for Structural Interpretation of Shock Metamorphic Effects}

Linking shock metamorphic features to the orientation of a shock wave almost always uses a number of assumptions that simplify the geometry and conditions of the shock wave. First, it is assumed that, on the scale of a rock sample, the shock wave in natural impacts can be directly compared to the shock wave produced in shock recovery experiments (Langenhorst and Hornemann, 2005). The results of shock recovery experiments directly or indirectly form the basis of all of shock barometry, whereby shock metamorphic effects are linked to formation at a specific shock pressure (Stöffler and Langenhorst, 1994; Grieve et al., 1996; French and Koeberl, 2010). In such an experiment, a shock wave is generated by the impact of an explosively accelerated flyer plate onto a sample container. A shock wave propagates downwards, away from the interface of the flyer plate and sample container directly into the sample (Figure 1a) (Langenhorst and Hornemann, 2005). The shock wave experienced by the sample has a planar front (except at the boundaries of the sample) and produces a uniaxial state of stress, with a longitudinal stress oriented perpendicular to the front, and transverse stresses that have a lower magnitude than the longitudinal stress, that are equal, and are oriented parallel to the front (Figure 1a). The front of the rarefaction wave that initiates the release of the material from the state of stress travels parallel to the shock front, and thus, the state of stress throughout a shock recovery experiment is always uniaxial compression, where the maximum principal compressive stress is oriented perpendicular to 
This is a post-print of an article published in ICARUS (doi: 10.1016/j.icarus.2021.114687).

the shock and rarefaction fronts. Maximum shear stress is resolved on any plane with a $45^{\circ}$ angle to the longitudinal stress. The strain produced in the sample is uniaxial flattening, where the maximum shortening of the sample is parallel to the propagation direction of the shock and rarefaction waves and therefore, parallel to the maximum principal stress that the sample experienced. Thus, on the bulk scale the sample experiences coaxial deformation. On the small scale, shock metamorphic effects within the sample may individually express noncoaxial deformations (e.g., FFs, basal PDFs, or mineral twins), however the net effect of these individual deformations is to produce a net system that accommodates coaxial deformation on the bulk scale. Studies of shock metamorphism, (e.g., Milton et al., 1996a; Ebert et al., 2021) have therefore assumed, implicitly or explicitly, that the maximum principal compressive stress and the maximum finite shortening strains are always oriented perpendicular to the shock front of a natural shock wave.

Second, it is usually assumed that the origin of the shock wave in a natural impact can be approximated to be a point source (Holsapple, 1980) about which the shock wave spreads spherically and such that maximum compressive stresses during shock metamorphism are radial to that point at all locations (Figure 1b). In other words, shock metamorphic indicators of maximum principal stress/strain in a specific location will be oriented radially and parallel to each other (prior to any subsequent deformation during excavation and modification).

Finally, it is often assumed that shock metamorphism occurs at peak pressure, however, several examples exist of shock metamorphic features cross-cutting each-other (Poelchau and Kenkmann, 2011; Cox et al., 2018), indicating temporal sequences of shock metamorphism while the Hugoniot Elastic Limit (HEL) is exceeded. Consequently, if the assumption that the shock and rarefaction waves are parallel is an oversimplification, then differently oriented stress fields in the shock wave may cause structures that "point" in different orientations depending on their time of formation. Indeed, the orientation of 
This is a post-print of an article published in ICARUS (doi: 10.1016/j.icarus.2021.114687).

principal stresses and particle velocities in a natural impact are expected to rotate over the course of a shock pulse even when the shock front perfectly originates from a point and spreads spherically (Figure 1b). In this case, a rarefaction wave is generated by the reflection of the shock wave on the free surface, thus for any location that does not lie directly beneath the point source, the front of the shock wave is not parallel to the front of the rarefaction wave. As the shock front accelerates material outwards along its propagation path, and the rarefaction wave accelerates material inwards along its propagation path, the result is an excavation flow that curves upwards towards the surface with increasing distance from the point of impact (Figure 1b; Gault et al., 1968). 
This is a post-print of an article published in ICARUS (doi: 10.1016/j.icarus.2021.114687).

a)

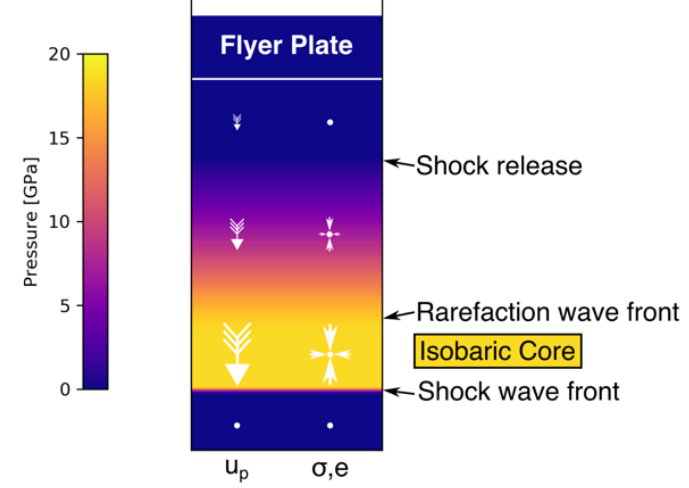

b)

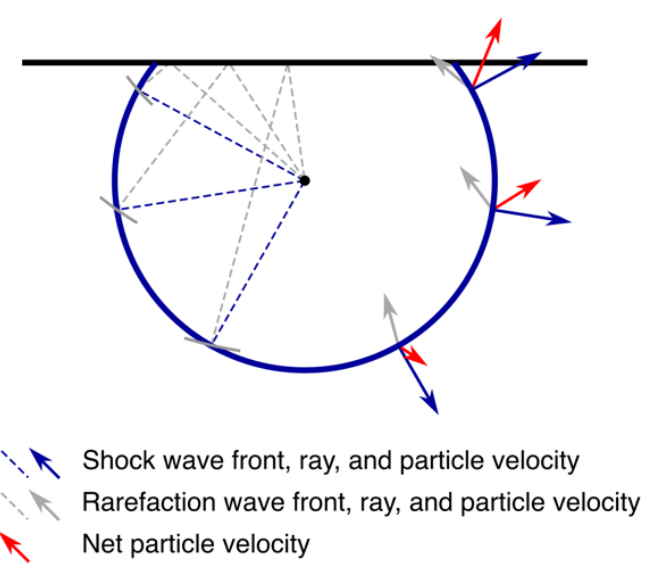

Figure 1: Shock waves in experiments and nature. a) Idealized shock recovery experiment where a flyer plate impacts a target material generating a planar wave that travels downwards into the target and is followed by a parallel and planar rarefaction wave. This produces orientations of particle velocity $\left(\mathrm{u}_{\mathrm{p}}\right)$, compressive stress $(\sigma)$, and strain $(\mathrm{e})$ that are parallel or orthogonal to each other and the shock front. b) Particle velocity rotation as a consequence of shock and rarefaction waves in a natural impact (Gault et al., 1968; based on Collins, pers. comm. and Melosh, 1984). In this conceptual model, a shock wave is generated at a point beneath the surface and radially expands, accelerating material away from the point source. When the shock wave reaches the free surface, a rarefaction wave is generated, directed back into the target. The rarefaction wave causes deceleration of the material along its propagation path, and a net post-shock velocity field that curves upwards towards the surface (i.e. the excavation flow). The orientation of stresses can be considered analogous to particle velocities (Gash, 1971). Close to the free surface, the interference of the shock and rarefaction waves mean that pressures never exceed the Hugoniot Elastic Limit. 
This is a post-print of an article published in ICARUS (doi: 10.1016/j.icarus.2021.114687).

In this study, we use numerical impact simulations to provide a quantitative description of how stresses and strains are oriented during the passage of the shock and rarefaction waves in an impact event. By doing this, we demonstrate where the commonly made assumptions are valid and where they are not. We hope that this demonstration will guide field studies in impact craters and facilitate improved interpretations of the products of shock metamorphism, highlighting the importance of the location of material in the target, and of establishing the exact timing of deformation during shock.

\section{Methods}

The shock physics code iSALE is a multi-rheology, multi-material code to study hypervelocity impacts. iSALE is based on the SALE hydrocode (Amsden et al., 1980) but has been modified to include an elasto-plastic constitutive model, fragmentation models (Melosh et al., 1992), various equations of state (Ivanov et al., 1997), a porosity compaction model (Wünnemann et al., 2006), and a dilatancy model (Collins, 2014). iSALE is a versatile code that can be used to model a variety of shock physics phenomena. In this study, we use iSALE to illustrate the behavior of principal stresses and strains during shock deformation.

To produce a generalized description of how stress and strain behave during shock metamorphism in planetary impacts, we employ a simple geometry, where a sphere impacts a flat target, perpendicular to the surface. The resolution of an impact simulation is typically defined in terms of the number of cells per projectile radius, in this study, we used 20 cells per projectile radius. In all simulations, we use an equation of state for granite (Pierazzo et al., 1998), generated by the analytic equation of state program (ANEOS; Thompson and Lauson, 1974) for both impactor and target. We note that similar results would be obtained for any rock-like equation of state. We use a constitutive model that describes the yield strength of a material as a function of pressure, damage, and temperature (Collins et al., 
This is a post-print of an article published in ICARUS (doi: 10.1016/j.icarus.2021.114687).

2004). For simplicity, we do not consider the role of porosity, dilatancy, or mechanical anisotropy in our models. In this study, we focus on a simulation with an impact velocity of $15 \mathrm{~km} / \mathrm{s}$ and an impactor diameter of $1 \mathrm{~km}$, which for the given resolution corresponds to a cell size of $25 \mathrm{~m}$. The size of the computational cell defines the length-scale at which the experienced conditions: pressure, temperature, stress, strain, etc. are considered homogenous. Different impactor velocities and impactor diameters were also tested to show how the results vary due to varying impact conditions (see Supplementary Material). Parameters used in our numerical simulations are shown in Table 1.

Shock waves are inherently nonlinear and undergo wave steepening to produce abrupt shock fronts that are often mathematically idealized as instantaneous jumps (Melosh, 1989). However, the finite difference methods employed by most shock physics codes, including iSALE, cannot accurately represent instantaneous jumps in field properties. Instead, the front of the shock wave must be distributed across several cells. This is achieved by the implementation of an "artificial viscosity" (see Anderson, 1987, for details) which acts during the compression of the material. In reality, shock fronts are never truly instantaneous as a result of shock viscosity (Swegle and Grady, 1985) and other rate-dependent processes. Strong shock waves in natural rocks may have fronts several meters in thickness (Melosh, 1989). The use of artificial viscosity in our simulations causes the width of a strong shock front, and therefore the strain rate within a strong shock front, to be controlled by the cell size. Artificial viscosity parameters are provided in Table $\mathbf{1 .}$ 
This is a post-print of an article published in ICARUS (doi: 10.1016/j.icarus.2021.114687).

Table 1. Material and global parameters for the numerical simulations.

\begin{tabular}{|c|c|}
\hline Parameter & Value \\
\hline Impactor Diameter, L (km) & $0.1,1,10$ \\
\hline Impactor Velocity, $\mathrm{v}_{\mathrm{i}}(\mathrm{km} / \mathrm{s})$ & $10,15,20$ \\
\hline Impact Angle $\left(^{\circ}\right)$ & 90 \\
\hline Surface Temperature (K) & 288 \\
\hline Surface Temperature Gradient $(\mathrm{K} / \mathrm{m})$ & 0.01 \\
\hline Resolution (Cells per impactor radius) & 20 \\
\hline Impactor and Target Material & Granite \\
\hline Poisson's Ratio & 0.3 \\
\hline Melting Temperature (K) & 1673 \\
\hline Simon Approximation - a & $6.0 \times 10^{9}$ \\
\hline Simon Approximation - c & 3.0 \\
\hline Thermal Softening Coefficient & 1.2 \\
\hline Intact Cohesion (MPa) & 10 \\
\hline Intact Coefficient of Internal Friction & 2.0 \\
\hline Damaged Cohesion (MPa) & 0.01 \\
\hline Damaged Coefficient of Internal Friction & 0.6 \\
\hline High Pressure Strength Limit (MPa) & 2500 \\
\hline Tensile Strength (MPa) & 1 \\
\hline Linear Artificial Viscosity Parameter & 0.24 \\
\hline Quadratic Artificial Viscosity Parameter & 1.2 \\
\hline
\end{tabular}


This is a post-print of an article published in ICARUS (doi: 10.1016/j.icarus.2021.114687).

iSALE uses an Eulerian frame of reference, where properties such as pressure, temperature, or stress, are stored on a fixed grid. Material is passed across the grid from timestep to timestep. To track the history of material in a simulation, a Lagrangian reference frame is more useful. Material packages are tracked in iSALE using Lagrangian tracer particles (Pierazzo et al., 1997). Here, the Lagrangian tracer particles save the locations, thermodynamic properties, and unique elements of the Cauchy stress tensor at each saved timestep. Strain is determined from the changes in location of tracer particles in a "pseudocell” between saved timesteps (Rae et al., 2019a). Strain can be calculated in one of two ways; using infinitesimal strain theory or finite strain theory. Infinitesimal strain theory is considerably simpler than finite strain theory but is inaccurate if the amount of solid-body rotation between the two points in time is large. Here, following the method described by (Rae et al., 2019a), we determine infinitesimal strain tensors by determining the deformation gradient tensor between each saved timestep; the increment of time between saved timesteps is sufficiently small that the pre-requisite of small rotations is maintained. The eigenvectors of the infinitesimal strain tensors correspond to the orientation of instantaneous stretching axes (ISAs). To ensure correspondence between the instantaneous stresses and the instantaneous strains that they cause, we linearly interpolate the Cauchy stress tensors of a pseudo-cell such that the analyzed stress tensors are centered within the cell and between saved timesteps. For further details on the method of determining the measures of stress and strain presented in this study, see the supplementary material of Rae et al. (2019a). General descriptions of stress and strain theory can be found by Fossen (2016) and Allmendinger et al. (2011).

Finite strain theory must be used to determine total strain tensors over large time intervals, where large rotations may occur. Here, we determine Lagrangian finite strain tensors between specific points in the shock wave to determine the principal strain 
This is a post-print of an article published in ICARUS (doi: 10.1016/j.icarus.2021.114687).

orientations during shock metamorphism. We divide the shock wave into two intervals; the first, which we term the "rise phase", is between the time where the pressure first exceeds the pressure at the HEL, $\mathrm{P}_{\mathrm{HEL}}$, and the time of peak pressure; the second, which we term the "release phase", is between the time of peak pressure and the time at which pressure drops below $\mathrm{P}_{\text {HEL }}$ (Figure 2).

In our results, all measures of stress and strain are shown based on their initial locations within the target. Solid-body rotations of the target material during shock propagation are negligibly small and are ignored. Subsequent movement and rotation of the material during the excavation and modification stages of impact cratering are not considered here. 
This is a post-print of an article published in ICARUS (doi: 10.1016/j.icarus.2021.114687).

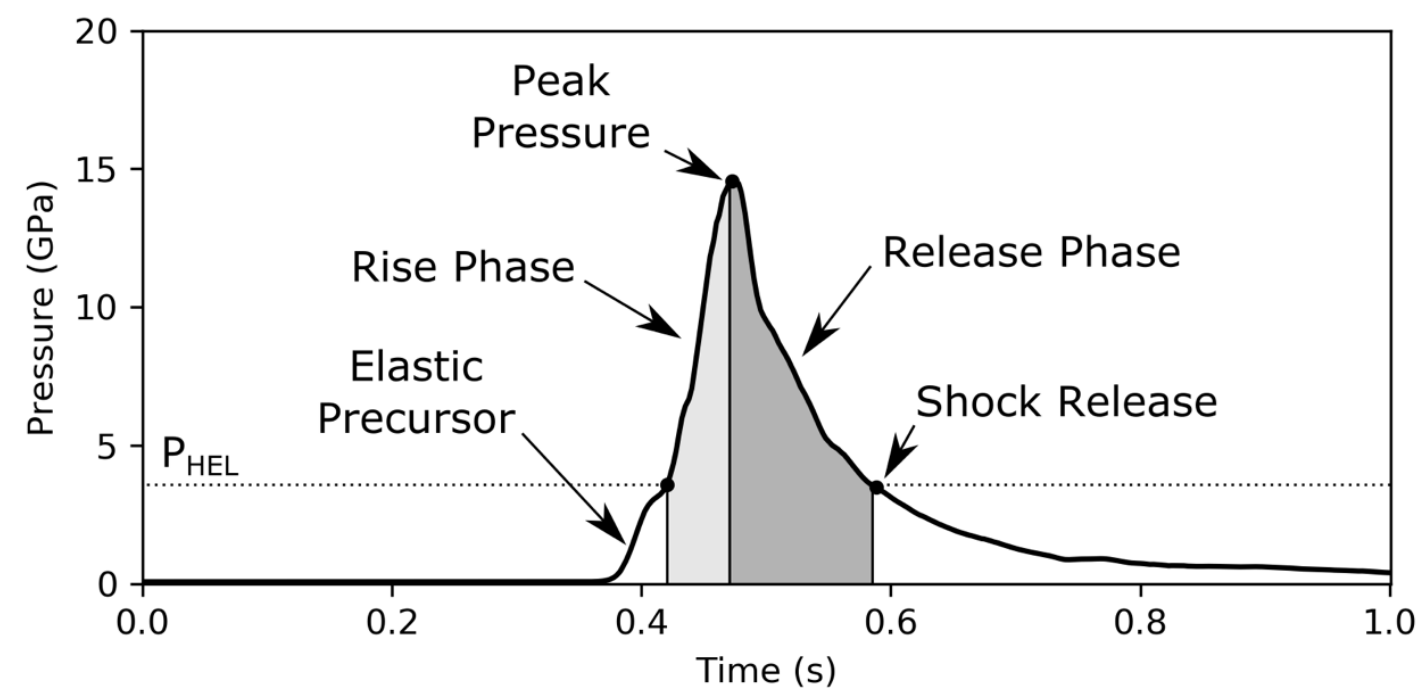

Figure 2. Pressure history of a shock wave. This shock wave has a maximum amplitude (peak pressure) of $\sim 15 \mathrm{GPa}$. The moments of peak pressure and shock release are indicated as points, while the rise phase and release phase are shown in light grey and dark grey shading, respectively. At this amplitude the velocity of the plastic shock wave is less than the elastic wave speed of the material and the plastic wave front is overtaken by an elastic precursor with an amplitude equal to the pressure at the Hugoniot Elastic Limit $\left(\mathrm{P}_{\mathrm{HEL}}\right)$. The shock front is not instantaneous as its name suggests, and instead has a finite rise time dependent on the coupling time of the projectile, and, in nature, rate-dependent failure processes, and, in numerical models, by the effect of artificial viscosity. This shock wave does not have an isobaric core because the rarefaction wave, which travels at a greater velocity than the plastic wave velocity, has caught up to the shock front. In all large impacts on Earth, where the minimum impact velocity is $11.2 \mathrm{~km} / \mathrm{s}$, all solid-state shock metamorphic effects occur by a shock wave without an isobaric core (Pierazzo et al., 1997). 
This is a post-print of an article published in ICARUS (doi: 10.1016/j.icarus.2021.114687).

\section{The Point Source Approximation}

Interpreting natural shock deformation and, more specifically, the orientations of shock metamorphic effects in the context of the dynamics of a shock wave is complex. A typical assumption is that some orientational measure of a shock metamorphic feature that is mapped about a crater will converge at some source near to the location of impact. The simplest and most commonly assumed source type is that of a point. Point source approximations have a long history in impact cratering, most notably in impact scaling to determine the "equivalent depth of burst" for analogous explosion craters and/or the origin of the excavation flow field. The simplest method to determine the equivalent depth of burst, $d$, is the jet-penetration formula (Birkhoff et al., 1948):

$$
\mathrm{d}=\mathrm{L}(\delta / \rho)^{1 / 2},(\mathbf{1})
$$

where $\mathrm{L}$ is the impactor diameter, and $\delta$ and $\rho$ are the densities of the impactor and target, respectively. Several other methods to determine the equivalent depth of burst have been developed (e.g. Baldwin, 1963; Dienes and Walsh, 1970; Bryan et al., 1978, 1980; Thomsen et al., 1979; Austin et al., 1980), however these methods are considerably more complex, require parameters that may not be readily known for natural impacts, and/or are notably inconsistent with observations at large velocities (see Holsapple, 1980). One final method to estimate the equivalent depth of burst derives from $\pi$-group scaling (Holsapple, 1980). For the conditions simulated here, the jet-penetration formula suggests the point source is at a depth $\mathrm{L}$ beneath the surface, while $\pi$-group scaling suggests a depth of $\sim 0.75 \mathrm{~L}$, varying slightly with impactor diameter and velocity (see Supplementary Material).

These methods all attempt to find a single, static, point beneath the target surface that represents the center of the cratering process. However, it has been noted that excavation 
This is a post-print of an article published in ICARUS (doi: 10.1016/j.icarus.2021.114687).

flow fields are best fitted by point sources that migrate with time (Austin et al., 1980; Anderson and Schultz, 2006). Despite these problems, point source approximations have considerable appeal due to their simplicity. In naturally shocked rocks, there is rarely a precise constraint on impactor speed, direction, and size; and in the case of shock metamorphism in meteorites, there is no constraint at all. Structural interpretations of shock metamorphic effects can only rarely account for the possibility of moving point sources and, to date, have never been done. Therefore, in this study we determine the orientation and magnitude of principal stresses along radial transects away from a static point source, which, for simplicity, we estimate using the jet-penetration formula (Equation 1).

\section{Magnitude and Orientation of Stress}

For the constitutive parameters of the granite used in the simulations, the pressure at the HEL, $\mathrm{P}_{\mathrm{HEL}}$, is $3.56 \mathrm{GPa}$, calculated as the pressure (the average of the three principal stresses) at which the elastic stress trajectory intersects the yield envelope (Melosh, 1989). The peak shock pressure required for the melting of granite is $\sim 60 \mathrm{GPa}$ (Stöffler et al., 2018). Here, we focus only on the region of the target that experiences shock pressures between $\mathrm{P}_{\mathrm{HEL}}$ and the peak pressure required for melting (Figure 3a), i.e. that experiences solid-state shock metamorphism. To illustrate how the orientation of principal stresses in the shock wave vary, we chose to measure the orientation of the maximum principal compressive stress at the time of peak shock pressure, and the time of shock release (Figure 3a). We have not represented the orientation of the maximum principal stress at the time where the HEL is first exceeded as it is generally parallel to the orientation of the maximum principal compressive stress at peak pressure (Supplementary Figure 2). 
This is a post-print of an article published in ICARUS (doi: 10.1016/j.icarus.2021.114687).
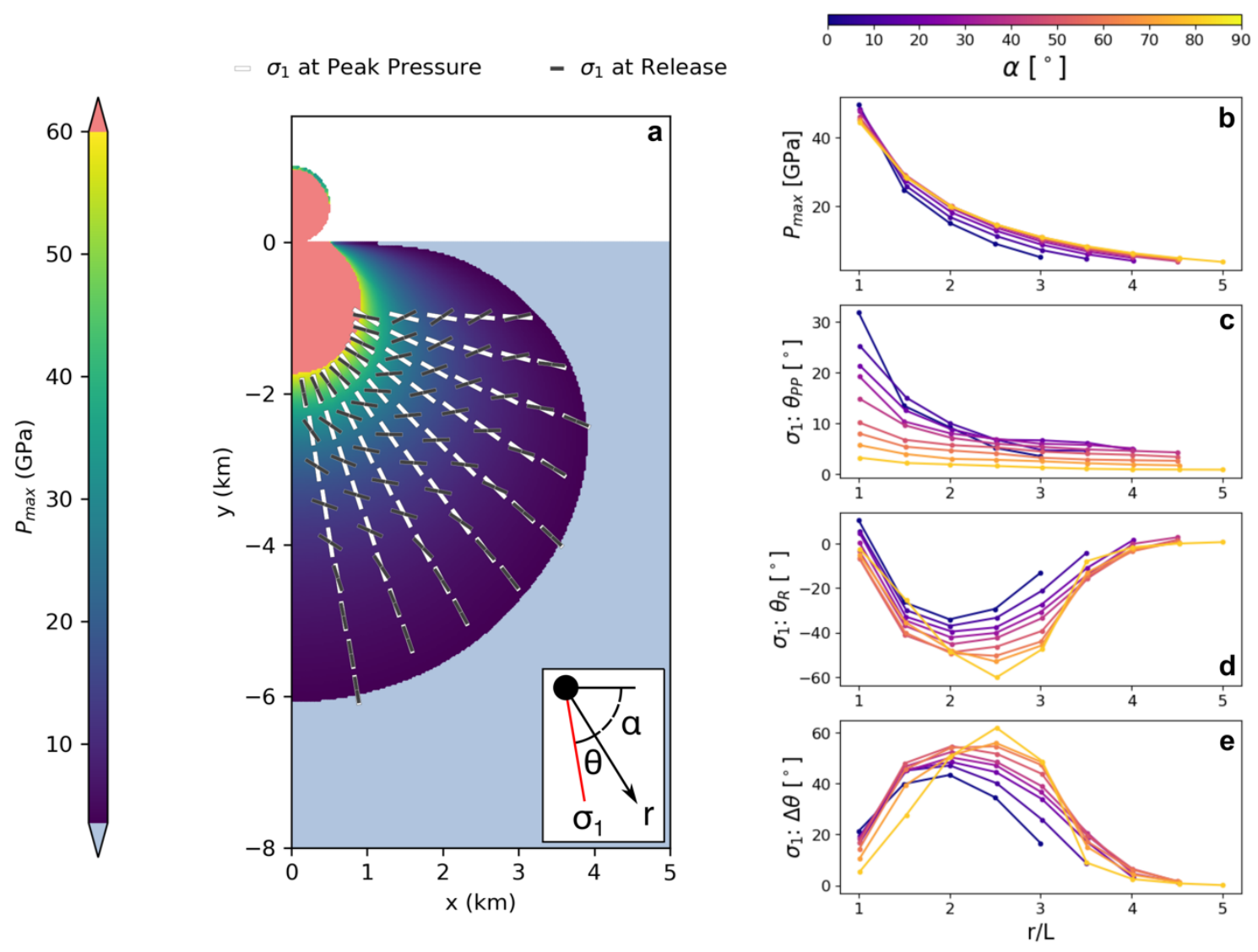

Figure 3. The orientation of principal stresses during shock metamorphism. (a) Lines indicate the orientation of maximum principal stress at peak pressure (white) and at shock release (grey). Material is plotted at its pre-impact location and is colored by peak shock pressure. (b-e) Radial trends (colored by transect plunge; $\alpha$ ) normalized by impactor diameter $(r / L)$ of (b) peak pressure, (c) the orientation of $\sigma_{1}$ at peak pressure relative to the radial direction $\left(\theta_{\mathrm{PP}}\right)$ where $0^{\circ}$ is a perfectly radial orientation, $(\mathrm{d})$ the orientation of $\sigma_{1}$ at the shock release relative to the radial direction $\left(\theta_{\mathrm{R}}\right)$ where $0^{\circ}$ is a perfectly radial orientation, and (e) the total amount of rotation of $\sigma_{1}$ between peak pressure and shock release $(\Delta \theta)$. The coordinate system used within the figure is shown on (a) where $r$ is the radial distance from the point source, $\alpha$ is the transect plunge, and $\theta_{\mathrm{PP}}$ and $\theta_{\mathrm{R}}$ are the angular deviation between radial and the $\sigma_{1}$ at peak pressure and shock release. 
This is a post-print of an article published in ICARUS (doi: 10.1016/j.icarus.2021.114687).

We find that the attenuation of shock pressures away from the point source is fairly similar between horizontal and near-vertical transects. Nevertheless, shock attenuates more rapidly horizontally than vertically (Figure 3b). In contrast, the orientation of $\sigma_{1}$ at peak pressure is only consistently close to being radial to the point source along steep transects. For shallower transects, radially directed $\sigma_{1}$ orientations are only achieved distally $(\mathrm{r} / \mathrm{L}>$ 2.5). Close to the point of impact, the maximum principal stress can be over $30^{\circ}$ steeper than radial (Figure 3c). The orientation of maximum principal compressive stresses at the release of the shock wave are more complex. Close to and far from the point of impact, the maximum principal compressive stresses are close to radial. At intermediate distances between $\sim 1.5$ and $3.5 \mathrm{r} / \mathrm{L}$ however, maximum principal compressive stresses at release can be between $30^{\circ}$ and $60^{\circ}$ shallower than radial (Figure 3d). In total, material at intermediate distances from the point of impact $(\sim 1.5-3 \mathrm{r} / \mathrm{L})$ experiences rotations of the maximum principal stress between $40^{\circ}$ (where $\alpha=0^{\circ}$ ) and $60^{\circ}$ (where $\alpha=80^{\circ}$ ) during shock metamorphism (Figure 3e). It is this region that experiences peak shock pressures between $\sim 10$ and $\sim 30 \mathrm{GPa}$, where most of the shock metamorphic features that have been associated with deviatoric stresses are known to form (French and Koeberl, 2010).

The shape of the stress ellipsoid also changes during shock metamorphism. While the three principal stresses are always compressional throughout shock metamorphism, the sign of the intermediate deviatoric stress $\left(\mathrm{s}_{2}=\sigma_{2}-\mathrm{P}\right)$ determines whether the stress ellipsoid is prolate $\left(s_{2}<0\right)$ or oblate $\left(s_{2}>0\right)$ (Figure 4). Along the horizontal transect, during the rise phase, $s_{2}$ is negative and is close to equal with $s_{3}$, i.e. the stress state is a prolate spheroid. However, after the rarefaction wave front arrives, coinciding with peak pressure, $\mathrm{s}_{2}$ diverges from $\mathrm{s}_{3}$. At locations close to the point source, where peak shock pressures are greater than $\sim 10 \mathrm{GPa}, \mathrm{s}_{2}$ eventually becomes positive, i.e. the stress ellipsoid changes from a prolate spheroid, through a prolate ellipsoid, to an oblate ellipsoid (Figure 5 and Supplementary 
This is a post-print of an article published in ICARUS (doi: 10.1016/j.icarus.2021.114687).

Videos 1-3). Further from the point of impact, where stresses are less than $\sim 10 \mathrm{GPa}$, the stress state only transitions from a prolate spheroid to a prolate ellipsoid (Figure 4 and Supplementary Videos 4 and 5). Along other transects, the same pattern can be observed where distal locations experience prolate stress states throughout shock metamorphism while close to the point of impact, material experiences oblate stress states during release of the shock wave (see Supplementary Material). At all times in the simulations here, $\sigma_{2}$ is oriented in the hoop direction. 
This is a post-print of an article published in ICARUS (doi: 10.1016/j.icarus.2021.114687).
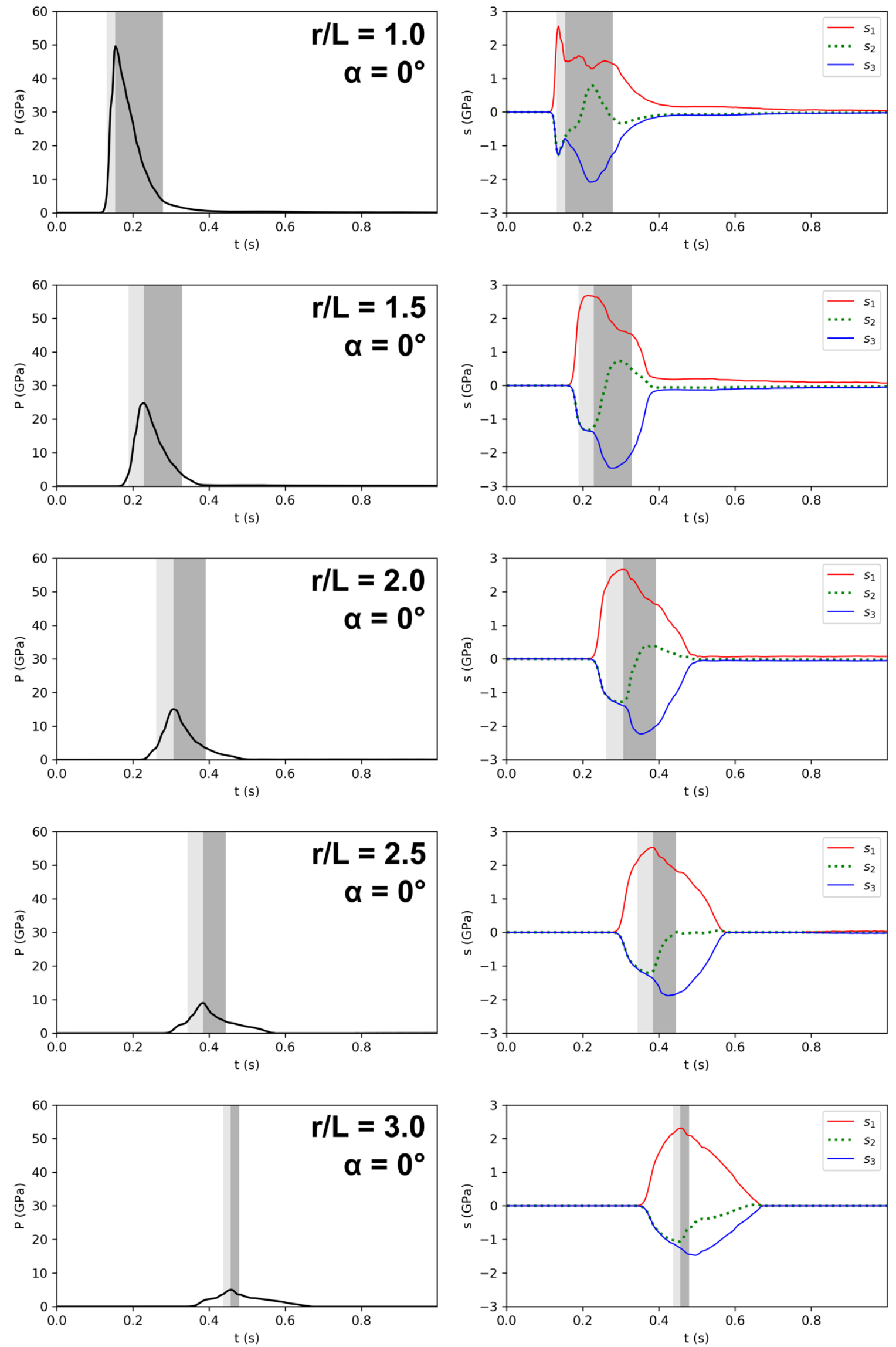
This is a post-print of an article published in ICARUS (doi: 10.1016/j.icarus.2021.114687).

Figure 4. Shock pressure and deviatoric stress history for the five tracer particles along the horizontal transect $\left(\alpha=0^{\circ}\right.$, Figure 3, see Supplementary Figures 2 and 3 for alternative transects), at increasing radial distances. The time intervals highlighted in light grey and dark grey show the intervals of the rise phase and release phase, respectively. 
This is a post-print of an article published in ICARUS (doi: 10.1016/j.icarus.2021.114687).
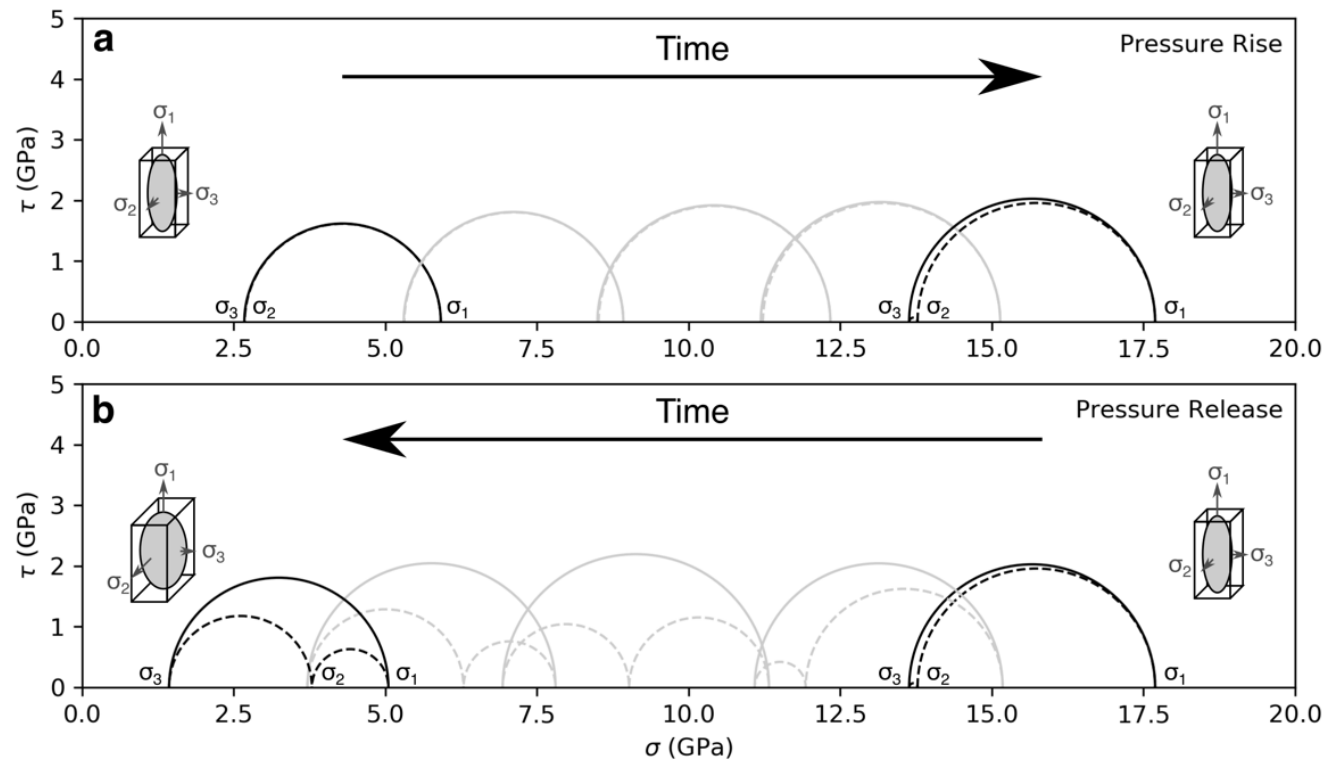

Figure 5. Mohr's circles during shock metamorphism for material initially at $\mathrm{r} / \mathrm{L}=2.0$ and $\alpha$ $=0^{\circ}$ (middle row of Figure 4). a) Stress states during the rise phase, where the stress states as the HEL is exceeded and peak pressure is reached are shown in black, intermediate states are shown in grey. Throughout this phase the stress state is an almost perfect prolate spheroid $\left(\sigma_{2}\right.$ $\left.=\sigma_{3}\right)$. b) Stress states during the release phase, stress states as peak pressure is reached and where pressures have been released back to the HEL are shown in black, intermediate states are shown in grey. In this phase the stress state is initially a prolate ellipsoid $\left(\sigma_{2} \approx \sigma_{3}\right)$ before becoming oblate ellipsoid $\left(\sigma_{2} \approx \sigma_{1}\right)$ as the release phase progresses. Stress states during shock metamorphism only become oblate at locations close to the impact point where pressures are less than $\sim 10 \mathrm{GPa}$ (see Supplementary Videos 1-5). 
This is a post-print of an article published in ICARUS (doi: 10.1016/j.icarus.2021.114687).

In addition to the simulation described so far, where the impactor has a diameter of 1 $\mathrm{km}$ and travels at $15 \mathrm{~km} \mathrm{~s}^{-1}$, simulations were run for varying impactor sizes and impactor velocities. We find that the radial variation of principal stress orientations is constant relative to the impactor size, i.e. that Figure 3b-e, where radial distance is plotted normalized to the impactor diameter, is accurate for all impactor diameters (relevant for terrestrial cratering, $100 \mathrm{~m}$ to $10 \mathrm{~km}$ ), where the impactor velocity is $15 \mathrm{~km} \mathrm{~s}^{-1}$ perpendicular to the target. Varying impact velocity causes some changes to the exact stress orientation at different times and locations during shock metamorphism. Most notably, increasing impactor velocity leads to greater volumes of shocked materials, therefore material that remains in the solid state is further from the point source and the distance at which the peak pressure in the shock wave decays beneath the HEL is also further from the point of impact, nevertheless the overall pattern remains where the rotation of the maximum principal stress between peak shock and release is $\sim 40-60^{\circ}$ (depending on the transect plunge) at intermediate distances from the point of impact (Supplementary Material). 
This is a post-print of an article published in ICARUS (doi: 10.1016/j.icarus.2021.114687).

\section{Magnitude and Orientation of Strain}

\section{Instantaneous Strain}

Instantaneous, or infinitesimal, strain describes how deformation progresses from an undeformed to a deformed state. Infinitesimal strain can be characterized by a variety of parameters, also known as "flow parameters". Here, we use instantaneous stretching axes (ISAs) and vorticity. ISAs are defined as the directions of maximum, minimum, and intermediate stretching rate at a given moment in time (Fossen, 2016), as such they are directly comparable with principal stress axes as they refer to the orientation of stretching at a given moment in time. ISAs are parallel to the principal orientations of the strain rate tensor, the "magnitude" of an ISA is therefore expressed as a strain rate. For a steady-state deformation, ISAs will remain at a constant orientation at all times.

Vorticity is a measure of the amount of rotation during deformation. Kinematic vorticity number $\left(\mathrm{W}_{\mathrm{k}}\right)$ describes the ratio of the strain rate to the internal vorticity during deformation (Fossen, 2016). $\mathrm{W}_{\mathrm{k}}=0$ corresponds to coaxial deformations such as pure shear, $\mathrm{W}_{\mathrm{k}}=1$ corresponds to rotational deformations such as simple shear, intermediate values correspond to a mixture of the two, commonly known as sub-simple shear.

Here, we show the orientation of ISA $_{3}$, the minimum instantaneous stretching axis (i.e. maximum instantaneous shortening), at the times of peak shock pressure (cf. Figure. 1) and at shock release (Figure 6), directly comparable to the orientations of maximum principal stress shown in Figure 3. 
This is a post-print of an article published in ICARUS (doi: 10.1016/j.icarus.2021.114687).
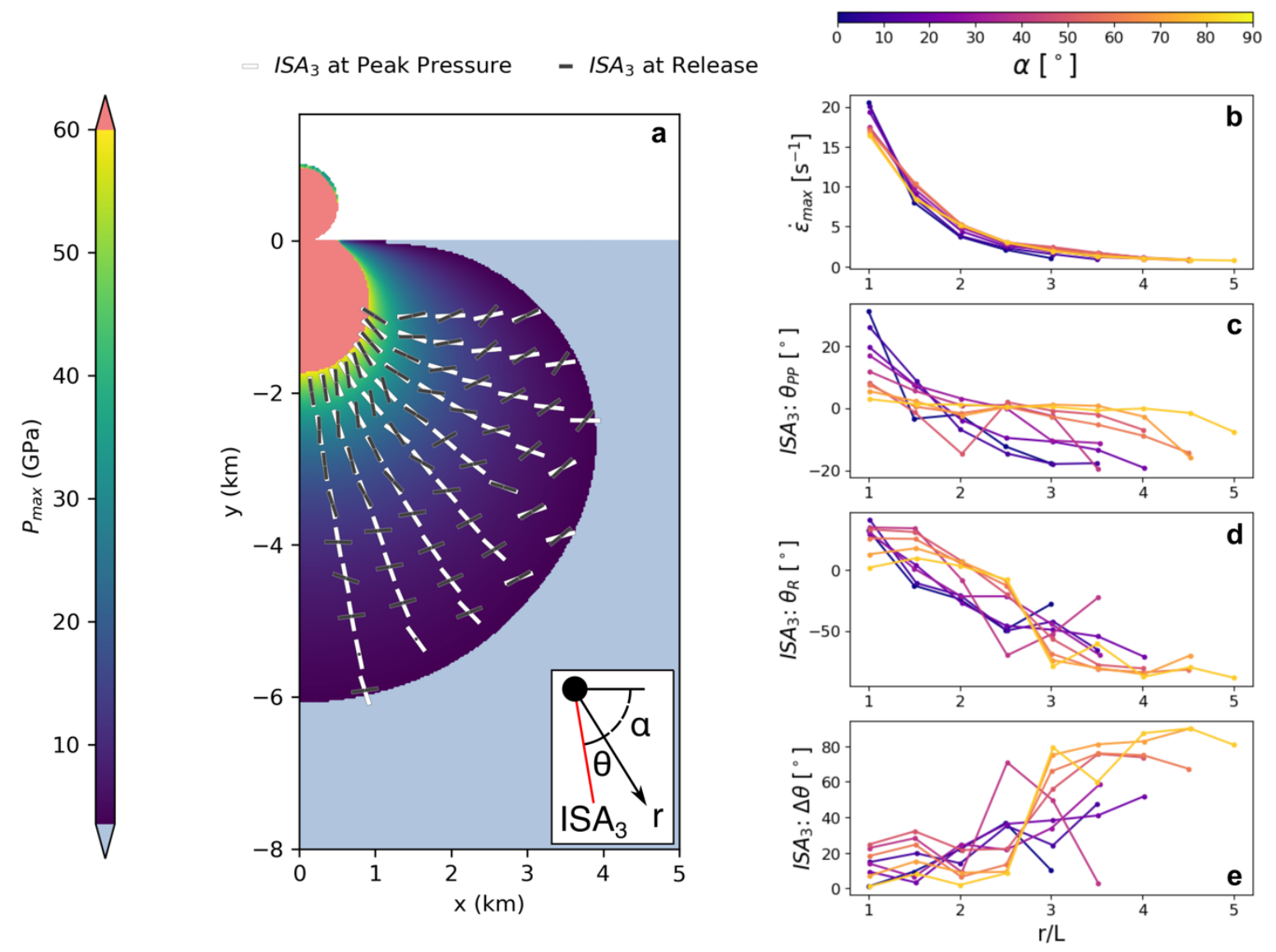

Figure 6. The orientation of instantaneous stretching axes (ISAs) during shock metamorphism. a) Lines indicate the minimum ISA (ISA 3 ) at peak pressure (white) and at shock release (grey). Material is plotted at its pre-impact location and is colored by peak shock pressure. (b-e) Radial trends (colored by transect plunge; $\alpha=0^{\circ}$ ) normalized by impactor size $(r / L)$ of (b) peak strain rate, (c) the orientation of $\mathrm{ISA}_{3}$ at peak pressure relative to the radial direction $\left(\theta_{\mathrm{PP}}\right)$ where $0^{\circ}$ is a perfectly radial orientation, $(\mathrm{d})$ the orientation of $\mathrm{ISA}_{3}$ at shock release relative to the radial direction $\left(\theta_{\mathrm{R}}\right)$ where $0^{\circ}$ is a perfectly radial orientation, and (e) the total amount of rotation of $\mathrm{ISA}_{3}$ between peak pressure and shock release $(\Delta \theta)$. The coordinate system used within the figure is shown on (a) where $r$ is the radial distance from the point source, $\alpha$ is the transect plunge, and $\theta_{\mathrm{PP}}$ and $\theta_{\mathrm{R}}$ are the angular deviation between radial and $\mathrm{ISA}_{3}$ at peak pressure and shock release, respectively. 
This is a post-print of an article published in ICARUS (doi: 10.1016/j.icarus.2021.114687).

We find that peak strain rates during shock metamorphism decay consistently away from the point source at all transect plunges, and, like the peak shock pressures, peak strain rate decays more rapidly in the horizontal direction than the vertical (Figure 6b). However, the absolute magnitudes of peak strain rates in our model can only be treated as approximate values, particularly for areas of the target that experience a strong shock wave ( $\mathrm{P}>\sim 35 \mathrm{GPa})$. Artificial viscosity causes strong shock fronts, where peak strain rates occur, to be spread over several cells of the computational grid; consequently the width of a grid cell acts to limit the peak strain rate. The orientation of $\mathrm{ISA}_{3}$ at peak stress is only consistently radial to the point source along near-vertical transects. In the horizontal direction, at locations close to the point of impact $\mathrm{ISA}_{3}$ plunges up to $25^{\circ}$ downwards relative to radial, while at locations far from the point of impact ISA 3 plunges up to $20^{\circ}$ upwards relative to radial (Figure $6 \mathbf{c}$ ).

The orientation of $\mathrm{ISA}_{3}$ at shock release follows a general trend of becoming more upwardly inclined, relative to radial, with increasing distance from the point of impact (Figure 6d). Close to the point source, $\mathrm{ISA}_{3}$ at shock release is directed radially only beneath the impact point; along the horizontal transect, $\mathrm{ISA}_{3}$ is directed $\sim 25^{\circ}$ downwards relative to radial. With increasing distance from the point source along the horizontal transect, the orientation of $\mathrm{ISA}_{3}$ gradually rotates towards angles of $\sim 60^{\circ}$ upwards. Along steeply inclined transects, the orientation of $\mathrm{ISA}_{3}$ remains fairly consistent and steeper than radial until, at $\sim 2.5 \mathrm{r} / \mathrm{L}$, orientations become perpendicular to radial. The consequence of this behavior is that $\mathrm{ISA}_{3}$ rotation during shock metamorphism can be up to $90^{\circ}$ at locations on near-vertical transects and far from the point of impact (Figure 6e). Close to the point of impact, ISA 3 rotation during shock metamorphism ranges from $0^{\circ}-25^{\circ}$. The orientations of ISAs as a function of normalized radial distance are independent of impactor size; some variations occur with varying impactor velocity, however, the overall trends remain (See

\section{Supplementary Material).}


This is a post-print of an article published in ICARUS (doi: 10.1016/j.icarus.2021.114687).

Deformation, i.e. strain, can be separated into two components: the hydrostatic/volumetric strain, with equal extension/contraction along all directions, and the deviatoric strain. The amount of volume strain experienced during shock passage is directly related to the mean stress, i.e. pressure. Rocks close to the point of impact experience greater pressures and therefore greater peak volume strains during shock metamorphism. For nonporous rocks, such as the granite modelled here, that experience shock pressures near to 60 GPa, the volume of rock contracts by $\sim 50 \%$ within the shock wave (Figure 7). Rocks that experience shock pressures near to $\mathrm{P}_{\mathrm{HEL}}$ only experience $\sim 5 \%$ volume contraction within the shock wave (Figure 7). The volumetric strains that are acquired up to peak pressure are fully recovered during pressure release, and the final volume after shock deformation is larger, by up to $\sim 7 \%$, than the original volume as a consequence of thermal expansion (Figure 7 ). An additional cause of volumetric expansion from shock metamorphism in nature is dilatancy, the volumetric expansion of granular materials as a consequence of shear strain, which has not been modeled here.

Large deviatoric strains accompany the hydrostatic strains experienced during shock deformation. Here, we present deviatoric instantaneous strains on modified Flinn-Ramsay diagrams (Fossen, 2016), demonstrating the path of deformation during shock metamorphism. As the diagrams represent the instantaneous strain states, the distance from the origin provides an overall measure of the strain rate at that moment of the deformation. Any strain state that plots on the 1:1 line is an instantaneous plane strain. Strain states that plot beneath the 1:1 line correspond to oblate (i.e. flattening) strains while states that plot above the 1:1 line correspond to prolate (i.e. constrictional) strains. During the rise phase, strain states are always flattening, oblate strains $\left(\dot{S}_{1} \approx \dot{S}_{2}>>\dot{S}_{3}\right.$; Figure 7). Towards the end of the rise phase, $\dot{S}_{2}$ begins to deviate from $\dot{S}_{1}$ producing a strain state that is still a flattening strain, though closer to plane strain than earlier deformation during the rise phase. During the 
This is a post-print of an article published in ICARUS (doi: 10.1016/j.icarus.2021.114687).

release phase $\dot{S}_{2}$ becomes progressively more similar to $\dot{S}_{3}$ resulting in the transformation from flattening strain states through plane strain $\left(\dot{S}_{1}>\dot{S}_{2}>\dot{S}_{3}, \dot{S}_{2} \approx 0\right)$ towards extremely constrictional, prolate strain states $\left(\dot{S}_{1}>>\dot{S}_{2} \approx \dot{S}_{3}\right.$; Figure 7). Close to the point of impact, flattening strains during pressure rise accumulate at a much greater rate than the constrictional strain during pressure release. With increasing distance from the point source, the rates of strain during the rise and release phases become more similar (Figure 7). 
This is a post-print of an article published in ICARUS (doi: 10.1016/j.icarus.2021.114687).
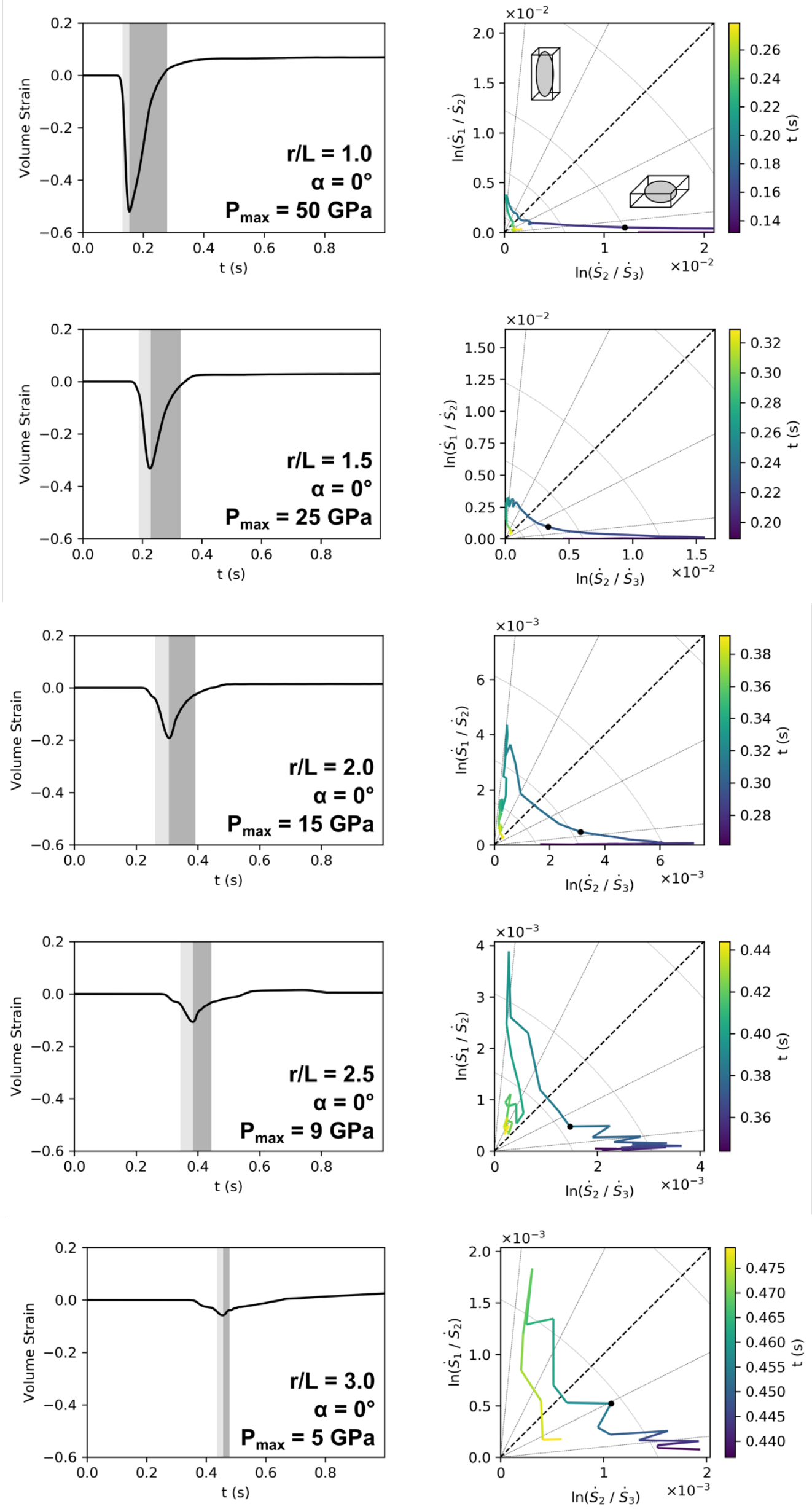
This is a post-print of an article published in ICARUS (doi: 10.1016/j.icarus.2021.114687).

Figure 7. Volumetric strain and stretching rate history for the five tracer particles along the horizontal transect $\left(\alpha=0^{\circ}\right.$, Figure 3 and 4, see Supplementary Figures 4 and 5 for alternative transects), at increasing radial distances. Left: Volumetric strain history. The time intervals highlighted in light grey and dark grey show the rise phase and release phase, respectively. Right: Modified Flinn-Ramsay diagrams of the instantaneous stretching rate ellipsoid over the rise and release phases, colored by time within that interval. The black point indicates the strain state at peak pressure. Increased distance from the origin indicates greater strain rates. Here, octahedral shear strain rate is contoured. Plane strain deformations plot along the 1:1 line $(\mathrm{k}=1$; dashed black line); flattening strains $(\mathrm{k}<1)$ plot beneath the line and constrictional strains $(\mathrm{k}>1)$ plot above the line. $\mathrm{k}$-values are contoured. Note the change in scale for the axes of the modified Flinn-Ramsay diagrams. 
This is a post-print of an article published in ICARUS (doi: 10.1016/j.icarus.2021.114687).

Deformation during cratering is characterized by kinematic vorticity numbers $\left(\mathrm{W}_{\mathrm{k}}\right)$ between 0 and 0.5 , i.e. deformation is either pure or sub-simple shear (Figure 8). We find that the average kinematic vorticity varies strongly between the rise and release phases. During the rise phase, small values of $\mathrm{W}_{\mathrm{k}}$ (near-coaxial deformations) can be found along steeply plunging transects from the point source, or distally along near horizontal transects. Moderate values of $\mathrm{W}_{\mathrm{k}}$ are experienced at moderate plunges and close to the point source along near-horizontal transects. During the release phase, small values of $\mathrm{W}_{\mathrm{k}}$ can be found proximally to the point source and along steeply plunging transects. Moderate values of $\mathrm{W}_{\mathrm{k}}$ are experienced distally along near-horizontal and moderately inclined transects. Thus, the only region of the crater subsurface that experiences small values of $\mathrm{W}_{\mathrm{k}}$ (near-coaxial deformations) throughout shock deformation is directly beneath the point source. All other regions of the subsurface experience some amount of rotational deformation during shock metamorphism. In addition, it is important to note that kinematic vorticity provides no measure of the changing orientation of the ISAs, i.e. whether or not the deformation is occurring under steady-state conditions, it describes internal rotation as an instantaneous property. Kinematic vorticity number as a function of normalized radial distance is independent of impactor size and impactor velocity (Supplementary Material). 
This is a post-print of an article published in ICARUS (doi: 10.1016/j.icarus.2021.114687).
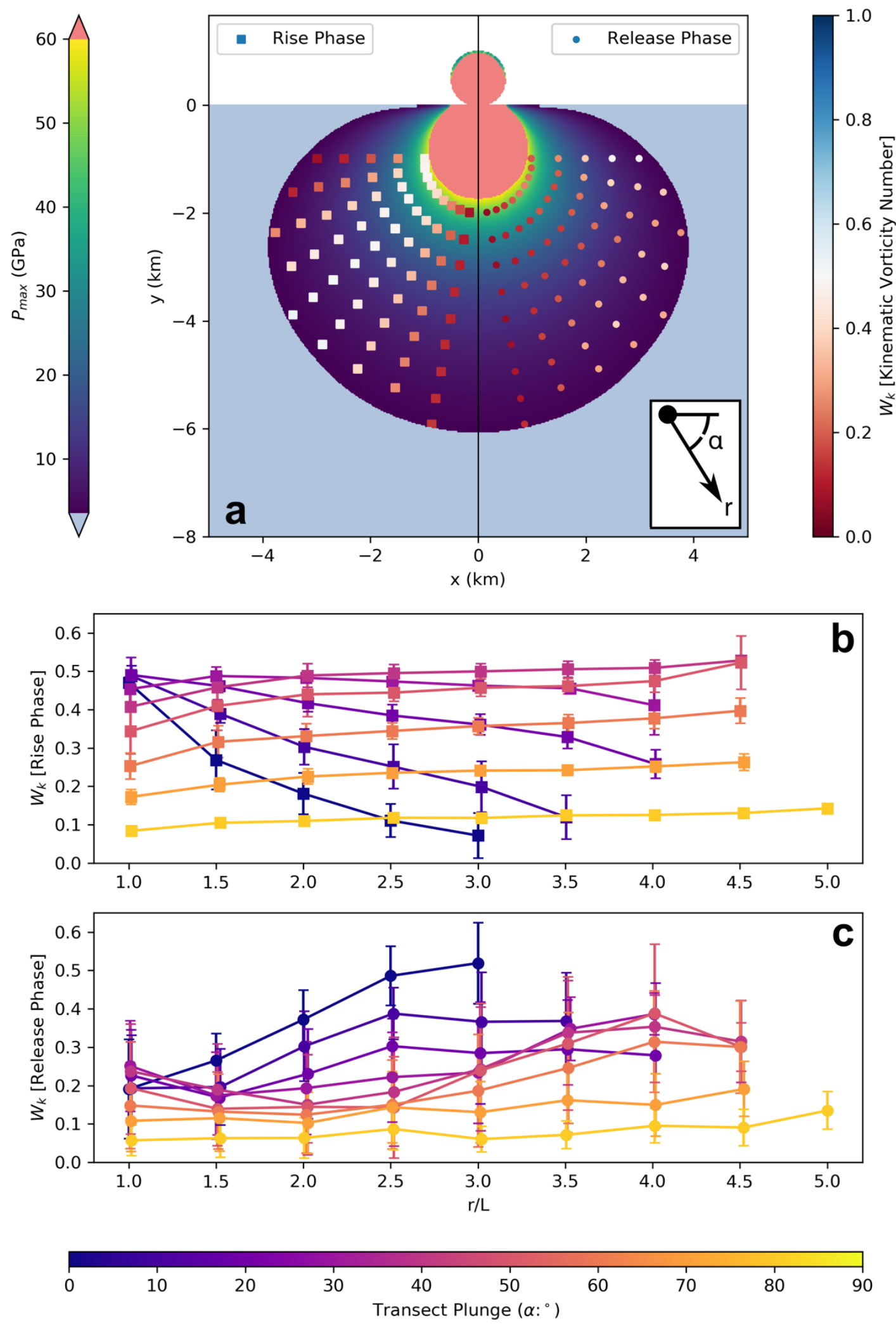
This is a post-print of an article published in ICARUS (doi: 10.1016/j.icarus.2021.114687).

Figure 8. Kinematic vorticity number $\left(\mathrm{W}_{\mathrm{k}}\right)$ during shock metamorphism. (a) Average kinematic vorticity number for material plotted at its original location. Square points are colored by the average kinematic vorticity during the rise phase while circular points are colored by the average kinematic vorticity during the release phase. The background is colored by peak shock pressure. The average kinematic vorticity number and one standard deviation error bars along transects at variable transect plunges for the rise phase (b) and the release phase (c). 
This is a post-print of an article published in ICARUS (doi: 10.1016/j.icarus.2021.114687).

\section{Finite Strain}

ISAs measure the orientation of stretching and shortening instantaneously, they can be directly compared to the principal stresses which also act as an instantaneous property. It is only under steady-state deformation that ISAs remain constant during a deformation, coaxial deformations can only occur under steady-state conditions. It has been demonstrated that deformation during impact cratering is rarely steady-state and consequently, the total deformation path is rarely coaxial. In a natural impact event, it is never possible to directly measure instantaneous properties such as principal stresses or ISAs that provide information of the deformation path, instead only the final state is known while the initial state can often be inferred. Consequently, it is only finite strain that is "seen" in the rock record. Finite strains are measured between an initial and a final state. Here, we present the orientation of the principal finite strains that accumulate during the rise phase and release phase (Figure 9a). In general, the axes of the minimum finite principal strain, $\mathrm{e}_{3}$, (which correspond to the maximum shortening axes) bend upwards, towards the surface, with increasing distance from the point source. Total shear strain accumulated during solid-state shock deformation decreases from $\sim 0.5$ with increasing distance from the point of impact (Figure $9 \mathbf{b}$,

Supplementary Material). Shear strain accumulated during the rise phase generally makes a greater contribution to the total shear strain than shear strain accumulated during the release phase. Close to the point of impact $\sim 75 \%$ of the total shear strain accumulated during shock deformation occurs during the rise phase. Far from the point of impact, the contribution to the total shear strain from deformation during pressure rise and pressure release are approximately equal (Figure 9a).

The orientation of $\mathrm{e}_{3}$ for the finite strain accumulated during the rise phase is only radial at locations close to the point of impact along near-horizontal transects; increasing radial distance and transect angle cause increasingly shallow orientations of $e_{3}$, up to $40^{\circ}$ 
This is a post-print of an article published in ICARUS (doi: 10.1016/j.icarus.2021.114687).

shallower than radial (Figure 9c). The orientation of $\mathrm{e}_{3}$ for the finite strain accumulated during the release phase is only close to radial at locations near to the point source. Along near-horizontal transects the orientation of $\mathrm{e}_{3}$ can rotate by up to $40^{\circ}$ to become significantly steeper than radial, near-vertical transects undergo less rotation of $\mathrm{e}_{3}$ with increasing radial distance but can still become significantly steeper than radial (Figure 9d). The net result of the orientations of $\mathrm{e}_{3}$ during the rise and release phases is that the orientation of $\mathrm{e}_{3}$ rotates by up to $\sim 30^{\circ}$ at moderate transect angles between pressure rise and release. The smallest rotation of $\mathrm{e}_{3}$ between pressure rise and pressure release occurs along near-horizontal transects. The greatest amount of rotation of $\mathrm{e}_{3}$ typically occurs at moderate distances from the point source (Figure 9e). The orientations of finite strains as a function of normalized radial distance are independent of impactor size and while some variations occur with varying impactor velocity, the overall trends remain (See Supplementary Material). 
This is a post-print of an article published in ICARUS (doi: 10.1016/j.icarus.2021.114687).
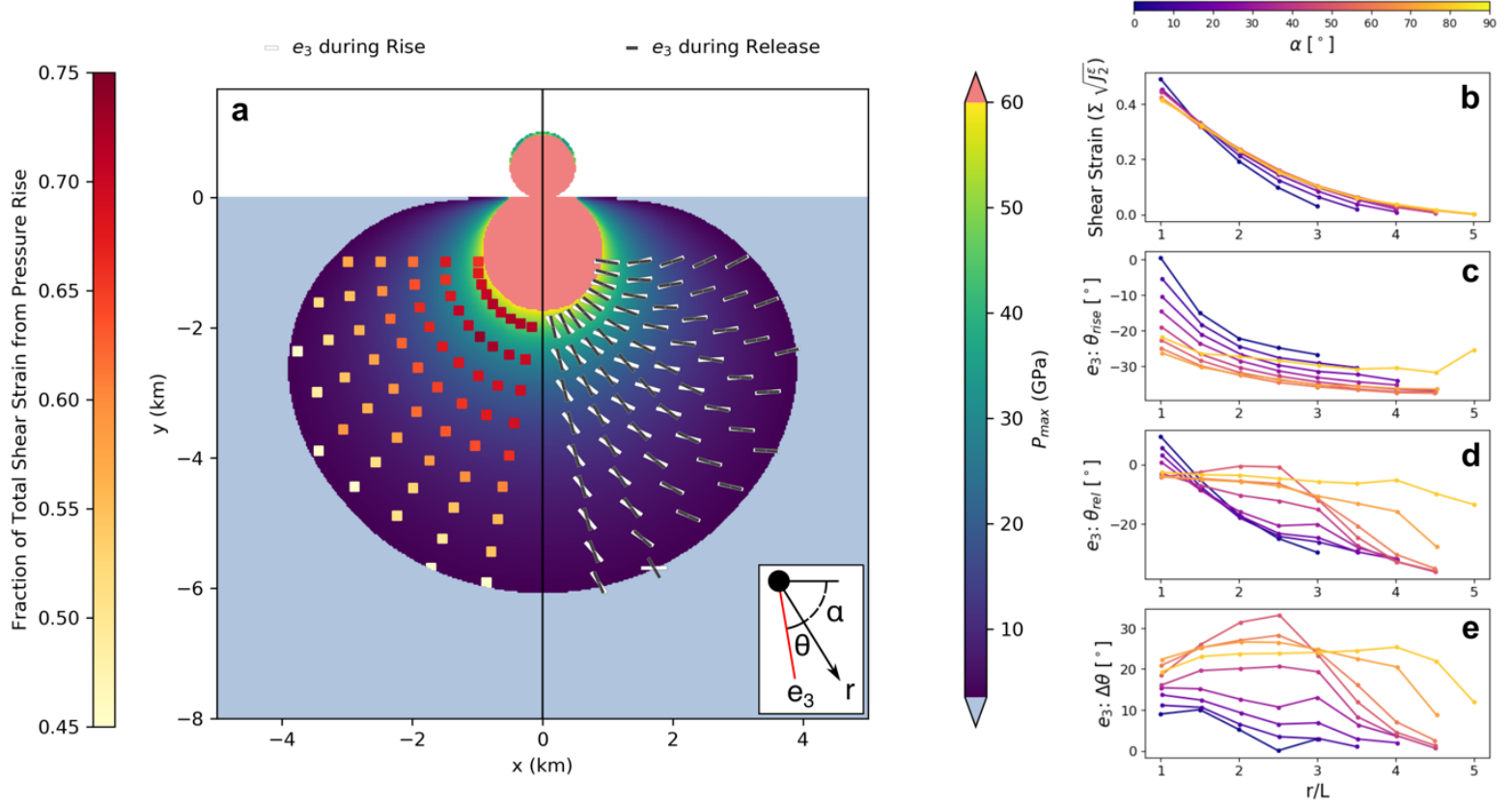

Figure 9. The orientation of minimum finite principal strains $\left(\mathrm{e}_{3}\right)$ during shock metamorphism. (a) Lines indicate $\mathrm{e}_{3}$ during the rise phase (white) and during the release phase (grey). Material is plotted at its original location and is colored by peak shock pressure. On the left, colored points show the proportion of the total shear strain accumulated during the rise phase as a fraction of the total shear strain accumulated during the rise and release phases. (b-e) Radial trends (colored by transect plunge; $\alpha$ ) normalized by impactor size $(r / L)$ of (b) total shear strain during shock metamorphism (determined from the instantaneous strain tensors), (c) the orientation of $\mathrm{e}_{3}$ during the rise phase relative to the radial direction $\left(\theta_{\text {rise }}\right)$ where $0^{\circ}$ is a perfectly radial orientation, (d) the orientation of $e_{3}$ during the release phase relative to the radial direction $\left(\theta_{\text {rel }}\right)$ where $0^{\circ}$ is a perfectly radial orientation, and (e) the total amount of rotation of $\mathrm{e}_{3}$ between the rise and release phases $(\Delta \theta)$. The coordinate system used within the figure is shown on (a) where $r$ is the radial distance from the point source, $\mathrm{L}$ is the impactor diameter, $\alpha$ is the transect plunge, and $\theta_{\text {rise }}$ and $\theta_{\text {rel }}$ are the angular deviation between radial and $\mathrm{e}_{3}$ at peak pressure and shock release. 
This is a post-print of an article published in ICARUS (doi: 10.1016/j.icarus.2021.114687).

\section{Discussion}

Fundamentally, the cause of rotation of the principal axes of stress and strain described here is the interaction of the shock and rarefaction waves. This concept has been explored previously in terms of the interaction of the particle velocities of the two waves to describe the shape of the excavation flow (Figure 1b; Gault et al., 1968). Furthermore, Gash (1971) proposed an analytical model to calculate stress interactions between shock and rarefaction waves as a mechanism for the formation of shatter cones. Those models make a number of simplifying assumptions, most notably that the shock wave expands spherically from a single point buried beneath the target surface, that the rarefaction wave is generated by the interaction of the shock wave with a perfectly elastic free-surface, and that the target is completely homogenous. Despite these simplifications, the results of those models bear a number of similarities to the analysis here, demonstrating the remarkable power of those early analytical models. Unfortunately, this concept has led to an impression that the shock wave can be treated as distinct from the rarefaction wave. For structural analysis of natural shock metamorphism, this is unhelpful; solid-state shock metamorphism occurs by a detached shock wave (Melosh, 1989), where the arrival of the rarefaction wave is coincident with the peak pressure, unlike shock recovery experiments, where samples are only investigated in the region where a shock wave with an isobaric core was present (Figure 1a; Langenhorst and Hornemann, 2005).

From the time of peak pressure, the rarefaction wave progressively alters the orientation of stress and instantaneous strain within the shock wave (Figure 10). As shock metamorphic effects form over a finite period of time, small though it may be, any feature that develops at or after the moment of peak pressure will experience some effect on its orientation due to the rarefaction wave. Stress and strain during the passage of a shock wave is never in steady-state (Figure 10) and is only close to being instantaneously coaxial in 
This is a post-print of an article published in ICARUS (doi: 10.1016/j.icarus.2021.114687).

specific locations within the target and during specific time intervals (Figure 9). This situation is distinctly unlike the shock recovery experiments against which shock metamorphic features are typically confirmed and calibrated for shock barometry (Figure 1a). 
This is a post-print of an article published in ICARUS (doi: 10.1016/j.icarus.2021.114687).
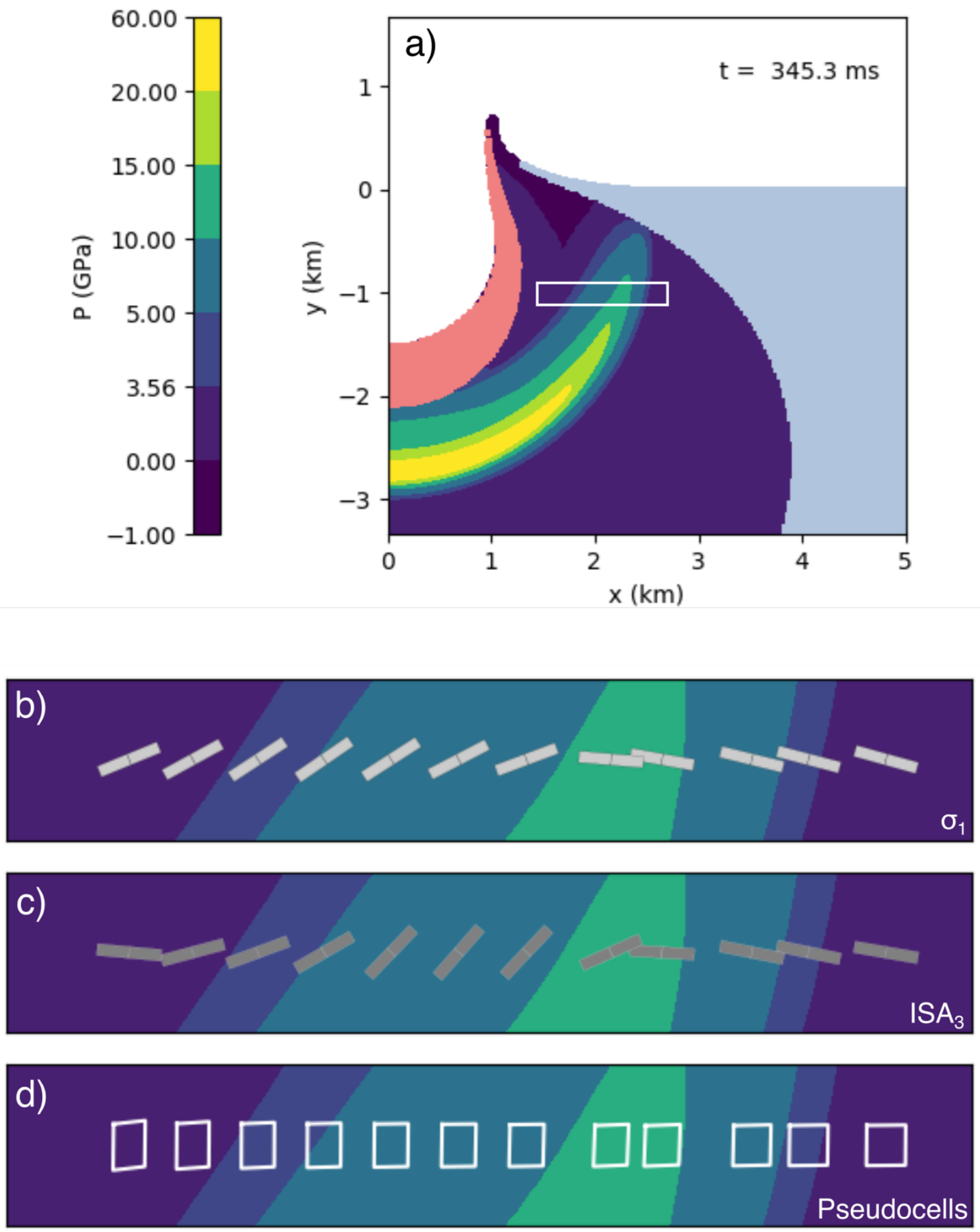
This is a post-print of an article published in ICARUS (doi: 10.1016/j.icarus.2021.114687).

Figure 10. Stress and strain profile through a natural shock wave. a) Crater growth resulting from a $1 \mathrm{~km}$ diameter impactor travelling at $15 \mathrm{~km} / \mathrm{s}$. Material is colored by the pressure field at $\mathrm{t}=345.3 \mathrm{~ms}$, where a detached shock wave can be seen. Material that experienced peak pressures above $60 \mathrm{GPa}$ is colored pink while material that will not experience peak pressures above the Hugoniot Elastic Limit is colored blue. b-d) Profiles through the simulated shock wave for 12 points $($ spacing $=0.1 \mathrm{~km}$ ) along a horizontal transect from the point source (inset on a). The three rows show b) the orientation of the maximum principal compressive stress $\left.\left(\sigma_{1}\right), c\right)$ the orientation of the minimum instantaneous stretching axis (ISA $)$, and d) the shape of the Lagrangian pseudocells, respectively. (The deformation between two locations provides a sense of the finite strain that occurs between two times within the shock wave). All points are affected by the stress wave, however the point ahead of the wave is affected only by the elastic precursor. 
This is a post-print of an article published in ICARUS (doi: 10.1016/j.icarus.2021.114687).

\section{Shatter Cones at the Gosses Bluff Impact Structure: A Case Study}

The results of this study have important implications for the interpretation of shock deformation features in impact structures in general. To illustrate this, we focus on a case study of shatter cone orientation at the Gosses Bluff impact structure, Northern Territory, Australia, as an example of how the results in this study can be used to guide the interpretation of shock deformation features.

Shatter cones are commonly suggested to be oriented radially, once reoriented to account for post-shock rotation, such that shatter cone apices converge on the point source of the shock wave (Dietz, 1961; Hargraves, 1961; Manton, 1965; Howard and Offield, 1968; Caty et al., 1976; Milton, 1977; Roddy and Davis, 1977; Stesky and Halls, 1983; Albat, 1988). The suggestion of purely radial orientations was originally related to the idea that shock-wave fronts are hemispherical in shape and that shatter cones would form with their apices oriented normal to that shock front (Milton, 1977). However, the mechanism of shatter cone formation is currently poorly understood (Baratoux and Reimold, 2016). Shatter cones were originally proposed to form prior to peak pressure, from the interaction of the elastic precursor of the shock wave with heterogeneities in the material (Johnson and Talbot, 1964; Dietz, 1968). However, spherules and melt films, sometimes vesiculated, containing shocked grains coating the fracture surface of some natural and experimental shatter cones supports the hypothesis of generation during compressional failure and potentially during pressure release, i.e. while pressures are above the $\mathrm{P}_{\mathrm{HEL}}$ (Gay, 1976; Gay et al., 1978; Gibson and Spray, 1998; Nicolaysen and Reimold, 1999; Pittarello et al., 2015; Wilk and Kenkmann, 2016). On the other hand, modeling studies have proposed that shatter cone formation occurs after pressures have dropped beneath the HEL, as a response to at least one of the principal stresses becoming tensile (Gash, 1971; Baratoux and Melosh, 2003; Sagy et al., 2004). A number of studies have also shown that shatter cone orientations are not always parallel at a 
This is a post-print of an article published in ICARUS (doi: 10.1016/j.icarus.2021.114687).

given locality (e.g. Milton et al., 1996a; Dressler et al., 1999; Wieland et al., 2006; Osinski and Ferrière, 2016); nevertheless these studies still generally attribute the orientation of shatter cones to the orientation of stresses during or shortly after shock and attribute the variable orientations of shatter cones to reflections of the shock wave in the target (e.g. Wieland et al., 2006; Osinski and Ferrière, 2016). The results of this study show that the stresses and strains experienced during an impact event vary both spatially within the target and temporally over the passage of the shock wave, even without reflections of the shock wave in the target. Different formation mechanisms therefore imply different orientations for shatter cones in natural impacts.

Gosses Bluff is a $\sim 24 \mathrm{~km}$ diameter central peak impact structure located on the northern margin of the Amadeus Basin of central Australia (Milton et al., 1996a). The exposed target rocks at Gosses Bluff are composed of a sequence of Ordovician to Devonian siliciclastic sedimentary rocks: sandstones, siltstones, and shales of generally shallow marine, fluvial, and occasionally aeolian origin (Wells et al., 1970; Shaw et al., 1991; Milton et al., 1996a). Measured on a regional scale, porosities in the target rocks range from negligible up to $\sim 20 \%$ in the sandstone units (Wells et al., 1970). Due to the presence of the central uplift, the present-day exposure is a bulls-eye pattern, where the oldest, and originally deepest, units are located in the center and the youngest, and originally shallowest, units are found on the peripheries (Milton et al., 1996a; Kenkmann et al., 2018). The diameter of the impactor required to form this structure has been estimated to be $\sim 2 \mathrm{~km}$ (Milton et al., 1996a).

Gosses Bluff is reknowned for its abundant shatter cones; whose orientations have been measured and analyzed in detail by Milton et al., (1996a). Their findings show that, once reoriented, shatter cone apices converge at a common latitude and longitude near the center of the structure. However, the focus of shatter cone apices, including consideration of relative stratigraphic height, do not converge at the same altitude (Figure 11). Instead, foci 
This is a post-print of an article published in ICARUS (doi: 10.1016/j.icarus.2021.114687).

from units in the center of the structure converge at an altitude $\sim 2000 \mathrm{~m}$ lower than foci from units further from the center. The altitudes of these foci are located between $\sim 2000 \mathrm{~m}$ and $\sim 4000 \mathrm{~m}$ above the present-day topographic surface. Milton et al. (1996a) attributed the variation of foci altitudes to inward displacement of material in the central uplift and used it to estimate that rocks exposed within the central peak must have reduced their mean horizontal radii by up to $52 \%$ during crater modification. In making this determination, Milton et al. (1996a) note that "the assumptions that the shock wave emanated from a point... and that it propagated as a spherical front are clearly simplifications" and that "no logical modifications would yield the observed pattern". In this study, we have shown how those assumptions can be modified to aid structural interpretations. 
This is a post-print of an article published in ICARUS (doi: 10.1016/j.icarus.2021.114687).

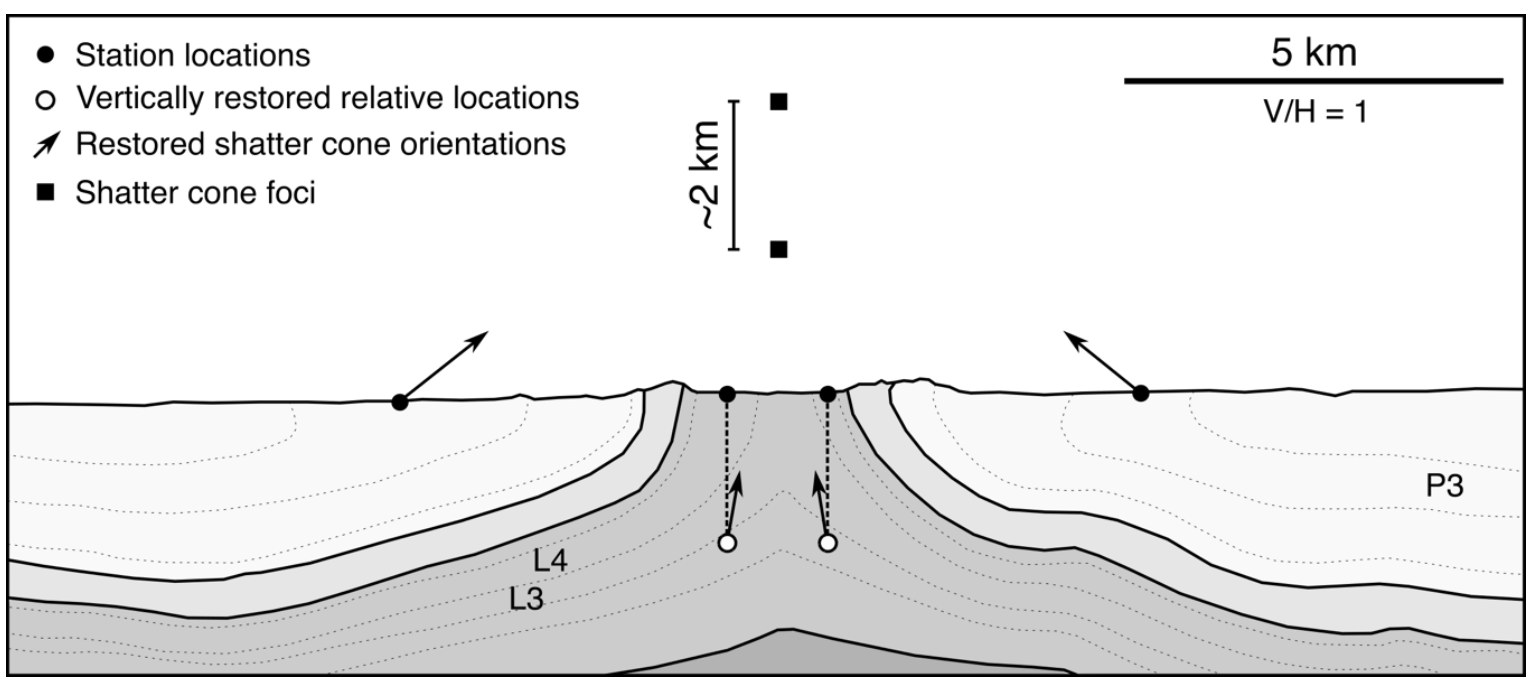

Figure 11: Shatter cone orientations at the Gosses Bluff impact structure. Schematic shatter cone locations (black points) in the Stairway Sandstone and Stokes Siltstone of the Larapinta Group (L3 and L4, respectively) and Hermannsburg Sandstone of the Pertnjara Group (P3) are shown. Stations are then vertically translated (relative to the height of the P3 station) such that their relative heights match their units' relative stratigraphic heights (white points). The shatter cone apices are then reorientated such that bedding is restored to its pre-impact orientation. Reoriented shatter cone axes are shown as black arrows, which converge at foci (black squares) located above the present-day surface. Deeper units, located in the center of the structure have their foci up to $\sim 2 \mathrm{~km}$ below the foci of units located at greater radial distances. Cross-section based on data and figures presented in Milton et al. (1996a, 1996b). 
This is a post-print of an article published in ICARUS (doi: 10.1016/j.icarus.2021.114687).

Let us first consider what the orientations of shatter cones at the Gosses Bluff structure would be if they formed with their apices parallel to the orientation of maximum principal stress. The unit located furthest from the center of the structure that contains shatter cones is the Hermannsburg Sandstone of the Pertnjara Group (P3; Figure 11), which was, at the time of impact, located at $\sim 1.5-2 \mathrm{~km}$ depth. Thus, the Hermannsburg Sandstone was originally located at $\sim 1$ impactor diameter beneath the surface. Exposure of the Hermannsburg Sandstone can be found $\sim 5 \mathrm{~km}$ from the center of the present-day structure. Assuming that these lithologies have only been transported a limited horizontal distance during crater modification, this corresponds to an original location at $\sim 2.5-3 \mathrm{r} / \mathrm{L}$.

Located near the center of the Gosses Bluff structure, the Stairway Sandstone and Stokes Siltstone units of the Larapinta Group (L3 and 4, respectively; Figure 11) were located $\sim 3-4 \mathrm{~km}(\sim 2 \mathrm{r} / \mathrm{L})$ beneath the surface at the time of impact. Additional constraint on the initial location of those rocks can be applied by considering shock metamorphism. Milton et al. (1996a) report that the most common orientation of PDFs in quartz from the center of the Gosses Bluff structure is $\{10 \overline{1} 3\}$ with the second-most abundant orientation of $\{10 \overline{1} 2\}$, suggesting, based on the shock calibration scheme of Hörz (1968), shock pressures of $\sim 20$ GPa. Furthermore, Milton et al. (1996a) report a lack of quartz polymorphs and very minor quantities of potential diaplectic quartz glass. On this basis, we estimate that the units close to the center of the present-day structure were located at $\sim 1.5-2 \mathrm{r} / \mathrm{L}$ (Figure 3b).

Having approximated the original locations of the shatter-cone bearing lithologies: Hermannsburg Sandstone at $\mathrm{r} / \mathrm{L}=3.0$ and $\alpha=0^{\circ}$ (where $\theta_{\mathrm{PP}}=\sim 5^{\circ}$ and $\theta_{\mathrm{R}}=\sim-10^{\circ}$; Figure 3), and the Stairway and Stokes units at $\mathrm{r} / \mathrm{L}=1.5$ and $\alpha=40^{\circ}$ (where $\theta_{\mathrm{PP}}=\sim 10^{\circ}$ and $\theta_{\mathrm{R}}=\sim-30^{\circ}$; Figure 3), the vertical difference between expected shatter cone foci, before any radial transport, can therefore be calculated applying simple trigonometry. If shatter cones form at peak pressure (or at any time during the rise phase), the expected difference between foci 
This is a post-print of an article published in ICARUS (doi: 10.1016/j.icarus.2021.114687).

altitude is $288 \mathrm{~m}$ (where the foci of the innermost lithologies are higher than the foci of the outermost lithologies). If shatter cones form at shock release, the expected difference between foci altitude is $464 \mathrm{~m}$ (where the foci of the innermost lithologies are now lower than the foci of the outermost lithologies). This means that, if shatter cones form early during shock deformation, the amounts of horizontal shortening estimated by Milton et al. (1996a) are underestimated by $\sim 15 \%$ and the foci of the shatter cone orientations are located $\sim 0.3$ impactor diameters above the estimated point source. If shatter cones form late during shock deformation, the amounts of horizontal shortening estimated by Milton et al. (1996a) are overestimated by $\sim 24 \%$ and the foci of shatter cone apices would be located over 0.5 impactor diameters beneath the estimated point source. Thus, the results presented in this study can be directly used to modify the assumptions made to interpret cratering processes from observations of shock deformation effects. Additionally, from the observation that shatter cone foci are located above the present day surface, this case study provides supporting evidence that shatter cones form early during shock deformation, inconsistent with models of shatter cone formation that invoke tensile stresses (Baratoux and Melosh, 2003; Sagy et al., 2004).

We note that the assumption that shatter cones form parallel to the maximum principal stress direction may not be correct. Shatter cones may instead be oriented parallel to the direction of maximum instantaneous shortening or finite stain. In this particular example, if shatter cones form early during shock parallel to instantaneous stretching axes, the amount of inward radial transport during modification must be greater still. In general, it may be important to consider what orientation shock deformation effects form parallel to, i.e. principal stresses, instantaneous strains, or finite strains over some interval of the shock wave duration. 
This is a post-print of an article published in ICARUS (doi: 10.1016/j.icarus.2021.114687).

In addition to the complexity of stress and strain orientation during the passage of the shock wave, target rocks in impact structures commonly undergo extreme deformation and rotation during crater excavation and modification (e.g. Rae et al., 2019a). Any attempt to relate the orientation of shock metamorphic features to the orientation of the stress wave must account for the change in orientation from this post-shock deformation and rotation. In practice, this requires that the original location of the material relative to the point source is known and is greatly aided if the original orientation of the material can be determined, e.g. bedding but also any other type of regional pre-impact foliation or lineation.

With this example, we hope to have demonstrated how the results of this study can be applied to the interpretation of shock deformation effects. We emphasize that these results apply generally to many shock deformation effects beyond shatter cones, such as feather features, shock twins, planar deformation features, and planar fractures. Indeed, it has been noted that shatter cones can be oriented in multiple directions at the same locality (Wieland et al., 2006; Osinski and Ferrière, 2016) and therefore may not form at a fixed orientation relative to the primary shock wave. For the purposes of this study though, this does not matter because we are using shatter cones as an example where the orientation of a shock deformation effect has been used, in general, to interpret crater mechanics, and more specifically, to infer amounts of shortening during crater collapse (Milton et al., 1996a). We have demonstrated how modifications to the assumption of point source behavior can improve those interpretations. Indeed, our results can and should be used to test whether shock deformation effects do have a fixed orientation relative to some measure of stress or strain in a shock wave. We would recommend a similar methodology be followed for any structural interpretation of a shock deformation effect, whether looking at the spatial variability of a shock deformation effect over a region of a crater or at the variability of shock effect orientation at a single locality or within a single sample. Either way, Figures 3, 6 or 9 
This is a post-print of an article published in ICARUS (doi: 10.1016/j.icarus.2021.114687).

can be used directly to determine how stress, instantaneous strain, or finite strain is oriented at any particular location and any particular time during shock.

\section{Validity of the point source approximation}

The results of this study demonstrate that the jet-penetration formula (Equation 1) provides an adequate description for the point from which peak shock pressures attenuate in an impact (Figure 3b), for the case, where the densities of the impactor and target are comparable. However, this point source, or indeed any point source approximation, cannot be used to describe a simple radial pattern of maximum principal stress during shock metamorphism (Figure 3a), even at the time of peak pressure (Figure 3c). Even if the source of the shock wave was at a static location (e.g., in explosion cratering), the orientations of principal stresses would still not be radial to that point throughout shock metamorphism because the rarefaction wave originates from the free surface producing an angular difference between the origin of the shock wave and the origin of the rarefaction wave (Figure 1b). Furthermore, the orientations of ISAs, particularly during the release phase, rotate by large amounts and are distinctly non-radial (Figure 6). This is further borne out by the orientations of finite strain during the rise and release phases (Figure 9). Thus, the interpretation that all locations experience stresses and/or strains that are radial with respect to an approximated point source is overly simplistic, nevertheless, as the point about which peak shock pressures attenuate radially and the point that marks the center of the impact structure, the concept of a point source remains a useful concept.

In addition, impactor obliquity may also cause deviation from the behavior described here. Nevertheless, impact craters are almost completely circular for all but the most oblique impacts $\left(<10-15^{\circ}\right)$, and crater ejecta remains completely symmetrical down to angles $<35$ $45^{\circ}$ (Kenkmann et al., 2014). As excavation is driven by the shock and rarefaction wave, a 
This is a post-print of an article published in ICARUS (doi: 10.1016/j.icarus.2021.114687).

symmetrical crater shape and ejecta distribution for all impacts steeper than $45^{\circ}$ indicates that this analysis is fully applicable to at least $50 \%$ of impact structures. Furthermore, peak shock distribution in oblique impacts is quite symmetrical and can still be approximated by a point source, albeit a point source that is displaced downrange of the point of impact (Pierazzo and Melosh, 2000; Kenkmann et al., 2014). Consequently, as the results presented here are given relative to the approximated point source, rather than the point of impact, we believe our results remain useful in the case of all but the most oblique impacts. A moving point source approximation would certainly be a more accurate description, however, at the current time, there is no general scaling relationship that describes point source migration as a function of impactor obliquity and velocity. Consequently, we would currently recommend that a static point source approximation is used for structural interpretation of shock metamorphic effects, albeit modified to consider the spatial and temporal variability of stress and strain orientation (Figures 3, 6, and 9).

\section{Spatial Resolution and Target Heterogeneity}

It is important to consider what stress and strain in a numerical simulation means in comparison to deformation in the natural world. Here, one grid cell is $25 \mathrm{~m}$ across. This is a scale much larger than the scale at which observations of shock metamorphism in minerals are made (i.e. the grain scale). The stress and deformation described here are not representative of what an individual grain experiences, instead it describes the bulk effect of the deformation of millions of grains, i.e. the rock mass. For example, a coaxial strain state in the rock mass may be the net effect of many, small-scale, non-coaxial strains produced by individual shock metamorphic effects arranged in conjugate sets.

Natural rocks are heterogeneous, and impedance mismatches between the heterogeneities in the target material are likely to cause locally variable stress orientations 
This is a post-print of an article published in ICARUS (doi: 10.1016/j.icarus.2021.114687).

and amplitudes; however, the impedance mismatches required to cause large reflections (within $50 \%$ of the peak pressure of the main shock) are greater than any common impedance contrast between typical planetary target materials and between minerals within a lithology (Stöffler et al., 1991). The only natural impedance mismatch large enough to cause significant reflections of the shock wave is between the rock-forming minerals and void spaces: pore spaces, open joints, fractures, etc. However, we note if the arrangement of pore spaces is homogeneous, the effects from local reflections on the orientation of shock metamorphism will produce scatter but, on the bulk scale, will average out to be consistent with the results presented here (Güldemeister et al., 2013).

\section{Conclusions}

To summarize, this study demonstrates:

1. That stress and strain orientations are spatially and temporally variable during solidstate shock metamorphism in natural impacts. This variability is, in general, not accurately described by radiality about a point source.

2. That the deformation path during shock metamorphism is unique to the location of the material relative to the point of impact.

3. The importance of understanding the exact conditions, and thus the time during a shock pulse, under which shock deformation effects form.

These factors have important implications for the orientation of shock effects such as shatter cones as well as numerous shear-derived shock microstructures. Without considering this aspect, shock metamorphic features cannot be related to the mechanics of a shock wave without uncertainty. Consequently, interpretations of shock deformation effects that relate to 
This is a post-print of an article published in ICARUS (doi: 10.1016/j.icarus.2021.114687).

larger scale cratering mechanics must account for the original location and orientation of material, together with subsequent cratering-related deformations. In this study, we have used a case example of the orientation of shatter cones at the Gosses Bluff impact structure to show how our analysis can be used to aid in the structural interpretation of shock metamorphic features in nature. Additionally, this case example demonstrates that the orientation of shatter cones in nature is consistent with formation during the earliest stages of shock deformation.

\section{Acknowledgements}

We gratefully thank the developers of the iSALE shock physics code: Jay Melosh, Boris Ivanov, Kai Wünnemann, Gareth Collins, Dirk Elbeshausen, and Tom Davison. Plots in this image were generated using the pySALEPlot tool developed by Tom Davison.

A.S.P.R. gratefully acknowledges invaluable discussions with Gareth Collins. We gratefully thank the reviewers, Roger Gibson and Nick Timms, and editor, Brandon Johnson, for their positive and constructive comments. This work was funded by DFG Project KE 732/27-1.

\section{References}

Agarwal, A., Kontny, A., Srivastava, D.C., Greiling, R.O., 2016. Shock pressure estimates in target basalts of a pristine crater: A case study in the Lonar crater, India. GSA Bulletin 128, 19-28. https://doi.org/10.1130/B31172.1

Agarwal, A., Poelchau, M.H., Kenkmann, T., Rae, A., Ebert, M., 2019. Impact experiment on gneiss: The effects of foliation on cratering process. Journal of Geophysical Research: Solid Earth 124, 13532-13546. https://doi.org/10.1029/2019JB018345

Albat, H.M., 1988. Shatter cone/bedding interrelationship in the Vredefort Structure: evidence for meteorite impact? South African Journal of Geology 91, 106-113. https://doi.org/10.10520/AJA10120750_937

Allmendinger, R.W., Cardozo, N., Fisher, D.M., 2011. Structural geology algorithms: Vectors and tensors. Cambridge University Press.

Amsden, A.A., Ruppel, H.M., Hirt, C.W., 1980. SALE: A simplified ALE computer program for fluid flow at all speeds (No. LA-8095). Los Alamos Scientific Lab., NM (USA). https://doi.org/10.2172/5176006 
This is a post-print of an article published in ICARUS (doi: 10.1016/j.icarus.2021.114687).

Anderson, C.E., 1987. An overview of the theory of hydrocodes. International Journal of Impact Engineering, Hypervelocity Impact Proceedings of the 1986 Symposium 5, 33-59. https://doi.org/10.1016/0734-743X(87)90029-7

Anderson, J.L.B., Schultz, P.H., 2006. Flow-field center migration during vertical and oblique impacts. International Journal of Impact Engineering, Hypervelocity Impact Proceedings of the 2005 Symposium 33, 35-44. https://doi.org/10.1016/j.ijimpeng.2006.09.022

Austin, M.G., Thomsen, J.M., Ruhl, S.F., Orphal, D.L., Schultz, P.H., 1980. Calculational investigation of impact cratering dynamics - Material motions during the crater growth period 11, 2325-2345.

Baldwin, R.B., 1963. The measure of the moon. University of Chicago Press.

Baratoux, D., Melosh, H.J., 2003. The formation of shatter cones by shock wave interference during impacting. Earth and Planetary Science Letters 216, 43-54. https://doi.org/10.1016/S0012-821X(03)00474-6

Baratoux, D., Reimold, W.U., 2016. The current state of knowledge about shatter cones: Introduction to the special issue. Meteoritics \& Planetary Science 51, 1389-1434. https://doi.org/10.1111/maps.12678

Birkhoff, G., MacDougall, D.P., Pugh, E.M., Taylor, S.G., 1948. Explosives with lined cavities. Journal of Applied Physics 19, 563-582. https://doi.org/10.1063/1.1698173

Bryan, J.B., Burton, D.E., Cunningham, M.E., Lettis, L.A., 1978. A two-dimensional computer simulation of hypervelocity impact cratering: Some preliminary results for Meteor Crater, Arizona 9, 128-130.

Bryan, J.B., Burton, D.E., Lettis, L.A., Morris, L.K., Johnson, W.E., 1980. Calculations of impact crater size versus meteorite velocity $11,112-114$.

Caty, J.-L., Chown, E.H., Roy, D.W., 1976. A new astrobleme: Ile Rouleau structure, Lake Mistassini, Quebec. Canadian Journal of Earth Sciences. https://doi.org/10.1139/e76085

Cavosie, A.J., Erickson, T.M., Timms, N.E., 2015. Nanoscale records of ancient shock deformation: Reidite ( $\mathrm{ZrSiO} 4)$ in sandstone at the Ordovician Rock Elm impact crater. Geology 43, 315-318. https://doi.org/10.1130/G36489.1

Collins, G.S., 2014. Numerical simulations of impact crater formation with dilatancy. Journal of Geophysical Research: Planets 119, 2600-2619. https://doi.org/10.1002/2014JE004708

Collins, G.S., Melosh, H.J., Ivanov, B.A., 2004. Modeling damage and deformation in impact simulations. Meteoritics \& Planetary Science 39, 217-231. https://doi.org/10.1111/j.1945-5100.2004.tb00337.x

Cox, M.A., Cavosie, A.J., Bland, P.A., Miljković, K., Wingate, M.T.D., 2018. Microstructural dynamics of central uplifts: Reidite offset by zircon twins at the Woodleigh impact structure, Australia. Geology 46, 983-986. https://doi.org/10.1130/G45127.1

Dienes, J.K., Walsh, J.M., 1970. Theory of impact: Some general principles and the method of Eulerian codes, in: High-Velocity Impact Phenomena. Elsevier, pp. 45-104.

Dietz, R.S., 1968. Shatter cones in cryptoexplosion structures, in: French, B.M., Short, N.M. (Eds.), Shock Metamorphism of Natural Materials. Mono Book Corp., Baltimore, pp. 87-99.

Dietz, R.S., 1967. Shatter cone orientation at Gosses Bluff astrobleme. Nature 216, 10821084. https://doi.org/10.1038/2161082a0

Dietz, R.S., 1961. Vredefort ring structure: Meteorite impact scar? The Journal of Geology 69, 499-516. https://doi.org/10.1086/626768 
This is a post-print of an article published in ICARUS (doi: 10.1016/j.icarus.2021.114687).

Dietz, R.S., 1947. Meteorite impact suggested by the orientation of shatter-cones at the Kentland, Indiana, disturbance. Science 105, 42-43. https://doi.org/10.1126/science.105.2715.42

Dietz, R.S., Butler, L.W., 1964. Shatter-cone Orientation at Sudbury, Canada. Nature 204, 280-281. https://doi.org/10.1038/204280a0

Dressler, B., 1990. Shock metamorphic features and their zoning and orientation in the Precambrian rocks of the Manicouagan Structure, Quebec, Canada. Tectonophysics, Cryptoexplosions and catastrophes in the geological record, with a special focus on the Vredefort structure 171, 229-245. https://doi.org/10.1016/0040-1951(90)90101-D

Dressler, B.O., Sharpton, V.L., Copeland, P., 1999. Slate Islands, Lake Superior, Canada: A mid-size, Complex Impact Structure. Presented at the Large Meteorite Impacts and Planetary Evolution, Geological Society of America.

Ebert, M., Poelchau, M.H., Kenkmann, T., Gulick, S.P.S., Hall, B., McCall, N., Rae, A.S.P., in press. Comparison of stress orientation indicators in Chicxulub's peak ring: Kinked biotites, basal PDFs, and feather features, in: Large Meteorites and Planetary Evolution VI. Geological Society of America Special Publications, pp. 1-XX.

Ebert, M., Poelchau, M.H., Kenkmann, T., Schuster, B., 2020. Tracing shock wave propagation in the Chicxulub Crater: Implications for the formation of peak rings. Geology.

Erickson, T.M., Cavosie, A.J., Pearce, M.A., Timms, N.E., Reddy, S.M., 2016. Empirical constraints on shock features in monazite using shocked zircon inclusions. Geology 44, 635-638. https://doi.org/10.1130/G37979.1

Erickson, T.M., Pearce, M.A., Reddy, S.M., Timms, N.E., Cavosie, A.J., Bourdet, J., Rickard, W.D.A., Nemchin, A.A., 2017. Microstructural constraints on the mechanisms of the transformation to reidite in naturally shocked zircon. Contrib Mineral Petrol 172, 6. https://doi.org/10.1007/s00410-016-1322-0

Fossen, H., 2016. Structural geology. Cambridge University Press.

French, B.M., 1998. Traces of catastrophe: A handbook of shock-metamorphic effects interrestrial meteorite impact craters. Lunar and Planetary Institute, Houston, Texas.

French, B.M., Koeberl, C., 2010. The convincing identification of terrestrial meteorite impact structures: What works, what doesn't, and why. Earth-Science Reviews 98, 123-170. https://doi.org/10.1016/j.earscirev.2009.10.009

Gash, P.J.S., 1971. Dynamic mechanism for the formation of shatter cones. Nature Physical Science 230, 32-35. https://doi.org/10.1038/physci230032a0

Gault, D.E., Quaide, W.L., Oberbeck, V.R., 1968. Impact cratering mechanics and structures, in: French, B.M., Short, N.M. (Eds.), Shock Metamorphism of Natural Materials. Mono Book Corp., Baltimore, pp. 87-99.

Gay, N.C., 1976. Spherules on shatter cone surfaces from the Vredefort structure, South Africa. Science 194, 724-725. https://doi.org/10.1126/science.194.4266.724

Gay, N.C., Comins, N.R., Simpson, C., 1978. The composition of spherules and other features on shatter cone surfaces from the Vredefort structure, South Africa. Earth and Planetary Science Letters 41, 372-380. https://doi.org/10.1016/0012-821X(78)901929

Gibson, H.M., Spray, J.G., 1998. Shock-induced melting and vaporization of shatter cone surfaces: Evidence from the Sudbury impact structure. Meteoritics \& Planetary Science 33, 329-336. https://doi.org/10.1111/j.1945-5100.1998.tb01637.x

Goltrant, O., Cordier, P., Doukhan, J.-C., 1991. Planar deformation features in shocked quartz; a transmission electron microscopy investigation. Earth and Planetary Science Letters 106, 103-115. https://doi.org/10.1016/0012-821X(91)90066-Q 
This is a post-print of an article published in ICARUS (doi: 10.1016/j.icarus.2021.114687).

Goltrant, O., Leroux, H., Doukhan, J.-C., Cordier, P., 1992. Formation mechanisms of planar deformation features in naturally shocked quartz. Physics of the Earth and Planetary Interiors 74, 219-240. https://doi.org/10.1016/0031-9201(92)90012-K

Graup, G., 1978. Das kristallin im Nördlinger Ries (PhD). Tübingen.

Grieve, R.A.F., Langenhorst, F., Stöffler, D., 1996. Shock metamorphism of quartz in nature and experiment: II. Significance in geoscience*. Meteoritics \& Planetary Science 31, 6-35. https://doi.org/10.1111/j.1945-5100.1996.tb02049.x

Güldemeister, N., Wünnemann, K., Durr, N., Hiermaier, S., 2013. Propagation of impactinduced shock waves in porous sandstone using mesoscale modeling. Meteoritics \& Planetary Science 48, 115-133. https://doi.org/10.1111/j.1945-5100.2012.01430.x

Hargraves, R.B., 1961. Shatter cones in the rocks of the Vredefort Ring. South African Journal of Geology 64, 147--161.

Holsapple, K.A., 1980. The equivalent depth of burst for impact cratering 11, 2379-2401. Hörz, F., 1968. Statistical measurements of deformation structures and refractive indices in experimentally shock loaded quartz, in: French, B.M., Short, N.M. (Eds.), Shock Metamorphism of Natural Materials. Mono Book Corporation, Baltimore, Maryland, pp. 23-54.

Hörz, F., Ahrens, T.J., 1969. Deformation of experimentally shocked biotite. Am J Sci 267, 1213-1229. https://doi.org/10.2475/ajs.267.10.1213

Howard, K.A., Offield, T.W., 1968. Shatter Cones at Sierra Madera, Texas. Science 162, 261-265. https://doi.org/10.1126/science.162.3850.261

Ivanov, B.A., Deniem, D., Neukum, G., 1997. Implementation of dynamic strength models into 2D hydrocodes: Applications for atmospheric breakup and impact cratering. International Journal of Impact Engineering, Hypervelocity Impact Proceedings of the 1996 Symposium 20, 411-430. https://doi.org/10.1016/S0734-743X(97)87511-2

Johnson, G.P., Talbot, R.J., 1964. A theoretical study of the shock wave origin of shatter cones (Master's). Air Force Insitute of Technology, Wright-Patterson Air Force Base, Ohio.

Kenkmann, T., Poelchau, M.H., Wulf, G., 2014. Structural geology of impact craters. Journal of Structural Geology 62, 156-182. https://doi.org/10.1016/j.jsg.2014.01.015

Kenkmann, T., Rae, A.S.P., Cavosie, A.J., Cox, M.A., Timms, N., Miljkovic, K., 2018. The Central Uplift of Gosses Bluff, Northern Territory, Australia 2067, 6077.

Kovaleva, E., Habler, G., 2019. Spatial distribution of zircon with shock microtwins in pseudotachylite-bearing granite gneisses, Vredefort impact structure, South Africa. Journal of Structural Geology 129, 103890. https://doi.org/10.1016/j.jsg.2019.103890

Kovaleva, E., Huber, M.S., Habler, G., Zamyatin, D.A., 2020. Zircon microstructures record deformation history of shock- and tectonically-generated pseudotachylites: A case study from the Vredefort Impact Structure, South Africa. J Petrology. https://doi.org/10.1093/petrology/egaa017

Langenhorst, F., Hornemann, U., 2005. Shock experiments on minerals: Basic physics and techniques, in: Miletich, R. (Ed.), Mineral Behaviour at Extreme Conditions, EMU Notes in Mineralogy. The Mineralogical Society of Great Britain and Ireland, pp. 357-387.

Leroux, H., Reimold, W.U., Koeberl, C., Hornemann, U., Doukhan, J.-C., 1999.

Experimental shock deformation in zircon: a transmission electron microscopic study. Earth and Planetary Science Letters 169, 291-301. https://doi.org/10.1016/S0012$821 \mathrm{X}(99) 00082-5$

Manton, W.I., 1965. The orientation and origin of shatter cones in the Vredefort Ring. Ann. N.Y. Acad. Sci 123, 1017-1049. 
This is a post-print of an article published in ICARUS (doi: 10.1016/j.icarus.2021.114687).

McLaren, A.C., Retchford, J.A., Griggs, D.T., Christie, J.M., 1967. Transmission electron microscope study of brazil twins and dislocations experimentally produced in natural quartz. physica status solidi (b) 19, 631-644.

https://doi.org/10.1002/pssb.19670190216

Melosh, H.J., 1989. Impact cratering: A geologic process. Oxford University Press.

Melosh, H.J., 1984. Impact ejection, spallation, and the origin of meteorites. Icarus 59, 234 260. https://doi.org/10.1016/0019-1035(84)90026-5

Melosh, H.J., Ryan, E.V., Asphaug, E., 1992. Dynamic fragmentation in impacts: Hydrocode simulation of laboratory impacts. Journal of Geophysical Research: Planets 97, 14735-14759. https://doi.org/10.1029/92JE01632

Milton, D.J., 1977. Shatter cones-an outstanding problem in shock mechanics, in: Impact and Explosion Cratering: Planetary and Terrestrial Implications. Pergamon Press, New York, pp. 715-750.

Milton, D.J., Glikson, A.Y., Brett, R., 1996a. Gosses Bluff-a latest Jurassic impact structure, central Australia. Part 1: geological structure, stratigraphy, and origin. AGSO Journal of Australian Geology and Geophysics 16, 453--486.

Milton, D.J., Barlow, B.C., Brown, A.R., Moss, F.J., Manwaring, E.A., Sedmik, E.C.E., Young, G.A., Son, J.V., 1996b. Gosses Bluff-a latest Jurassic impact structure, central Australia. Part 2: seismic, magnetic, and gravity studies. AGSO Journal of Australian Geology and Geophysics 16, 487--528.

Nakamura, T., Tomeoka, K., Sekine, T., Takeda, H., 1995. Impact-induced chondrule flattening in the Allende CV3 carbonaceous chondrite: Shock experiments. Meteoritics 30, 344-347. https://doi.org/10.1111/j.1945-5100.1995.tb01133.x

Nakamura, T., Tomeoka, K., Takaoka, N., Sekine, T., Takeda, H., 2000. Impact-induced textural changes of $\mathrm{CV}$ carbonaceous chondrites: Experimental reproduction. Icarus 146, 289-300. https://doi.org/10.1006/icar.2000.6385

Nicolaysen, L.O., Reimold, W.U., 1999. Vredefort shatter cones revisited. Journal of Geophysical Research: Solid Earth 104, 4911-4930. https://doi.org/10.1029/1998JB900068

Osinski, G.R., Ferrière, L., 2016. Shatter cones: (Mis)understood? Science Advances 2, e1600616. https://doi.org/10.1126/sciadv.1600616

Pierazzo, E., Kring, D.A., Melosh, H.J., 1998. Hydrocode simulation of the Chicxulub impact event and the production of climatically active gases. Journal of Geophysical Research: Planets 103, 28607-28625. https://doi.org/10.1029/98JE02496

Pierazzo, E., Melosh, H.J., 2000. Hydrocode modeling of oblique impacts: The fate of the projectile. Meteoritics \& Planetary Science 35, 117-130. https://doi.org/10.1111/j.1945-5100.2000.tb01979.x

Pierazzo, E., Vickery, A.M., Melosh, H.J., 1997. A reevaluation of impact melt production. Icarus 127, 408-423. https://doi.org/10.1006/icar.1997.5713

Pittarello, L., Ferrière, L., Feignon, J.-G., Osinski, G.R., Koeberl, C., 2020. Preferred orientation distribution of shock-induced planar microstructures in quartz and feldspar. Meteoritics \& Planetary Science n/a. https://doi.org/10.1111/maps.13490

Pittarello, L., Nestola, F., Viti, C., Crósta, A.P., Koeberl, C., 2015. Melting and cataclastic features in shatter cones in basalt from the Vista Alegre impact structure, Brazil. Meteoritics \& Planetary Science 50, 1228-1243. https://doi.org/10.1111/maps.12466

Poelchau, M.H., Kenkmann, T., 2011. Feather features: A low-shock-pressure indicator in quartz. Journal of Geophysical Research: Solid Earth 116. https://doi.org/10.1029/2010JB007803

Rae, A.S.P., Collins, G.S., Poelchau, M., Riller, U., Davison, T.M., Grieve, R.A.F., Osinski, G.R., Morgan, J.V., Scientists, I.-I.E. 364, 2019a. Stress-strain evolution during peak- 
This is a post-print of an article published in ICARUS (doi: 10.1016/j.icarus.2021.114687).

ring formation: A case study of the Chicxulub impact structure. Journal of Geophysical Research: Planets 124, 396-417. https://doi.org/10.1029/2018JE005821

Rae, A.S.P., Collins, G.S., Morgan, J.V., Salge, T., Christeson, G.L., Leung, J., Lofi, J., Gulick, S.P.S., Poelchau, M., Riller, U., Gebhardt, C., Grieve, R.A.F., Osinski, G.R., 2019b. Impact-induced porosity and microfracturing at the Chicxulub impact structure. Journal of Geophysical Research: Planets 124, 1960-1978. https://doi.org/10.1029/2019JE005929

Roddy, D.J., Davis, L.K., 1977. Shatter cones formed in large-scale experimental explosion craters, in: Impact and Explosion Cratering: Planetary and Terrestrial Implications. Pergamon Press, New York, pp. 715-750.

Sagy, A., Fineberg, J., Reches, Z., 2004. Shatter cones: Branched, rapid fractures formed by shock impact. Journal of Geophysical Research: Solid Earth 109. https://doi.org/10.1029/2004JB003016

Schedl, A., 2006. Applications of twin analysis to studying meteorite impact structures. Earth and Planetary Science Letters 244, 530-540. https://doi.org/10.1016/j.eps1.2006.02.018

Shaw, R.D., Etheridge, M.A., Lambeck, K., 1991. Development of the Late Proterozoic to Mid-Paleozoic, intracratonic Amadeus Basin in central Australia: A key to understanding tectonic forces in plate interiors. Tectonics $10,688-721$. https://doi.org/10.1029/90TC02417

Stesky, R.M., Halls, H.C., 1983. Structural analysis of shatter cones from the Slate Islands, northern Lake Superior. Canadian Journal of Earth Sciences. https://doi.org/10.1139/e83-001

Stöffler, D., Hamann, C., Metzler, K., 2018. Shock metamorphism of planetary silicate rocks and sediments: Proposal for an updated classification system. Meteoritics \& Planetary Science 53, 5-49. https://doi.org/10.1111/maps.12912

Stöffler, D., Keil, K., Edward R.D, S., 1991. Shock metamorphism of ordinary chondrites. Geochimica et Cosmochimica Acta 55, 3845-3867. https://doi.org/10.1016/00167037(91)90078-J

Stöffler, D., Langenhorst, F., 1994. Shock metamorphism of quartz in nature and experiment: I. Basic observation and theory*. Meteoritics 29, 155-181. https://doi.org/10.1111/j.1945-5100.1994.tb00670.x

Swegle, J.W., Grady, D.E., 1985. Shock viscosity and the prediction of shock wave rise times. Journal of Applied Physics 58, 692-701. https://doi.org/10.1063/1.336184

Thompson, S.L., Lauson, H.S., 1974. Improvements in the CHART D radiationhydrodynamic code III: revised analytic equations of state (No. SC-RR-710714). Sandia National Laboratory, Alberquerque, New Mexico.

Thomsen, J.M., Austin, M.G., Ruhl, S.F., Schultz, P.H., Orphal, D.L., 1979. Calculational investigation of impact cratering dynamics - Early time material motions 10, 27412756.

Timms, N.E., Erickson, T.M., Pearce, M.A., Cavosie, A.J., Schmieder, M., Tohver, E., Reddy, S.M., Zanetti, M.R., Nemchin, A.A., Wittmann, A., 2017a. A pressuretemperature phase diagram for zircon at extreme conditions. Earth-Science Reviews 165, 185-202. https://doi.org/10.1016/j.earscirev.2016.12.008

Timms, N.E., Healy, D., Erickson, T.M., Nemchin, A.A., Pearce, M.A., Cavosie, A.J., 2017b. Role of elastic anisotropy in the development of deformation microstructures in zircon, in: Microstructural Geochronology. American Geophysical Union (AGU), pp. 183-202. https://doi.org/10.1002/9781119227250.ch8

Timms, N.E., Pearce, M.A., Erickson, T.M., Cavosie, A.J., Rae, A.S.P., Wheeler, J., Wittmann, A., Ferrière, L., Poelchau, M.H., Tomioka, N., Collins, G.S., Gulick, 
This is a post-print of an article published in ICARUS (doi: 10.1016/j.icarus.2021.114687).

S.P.S., Rasmussen, C., Morgan, J.V., IODP-ICDP Expedition 364 Scientists, 2019. New shock microstructures in titanite (CaTiSiO5) from the peak ring of the Chicxulub impact structure, Mexico. Contrib Mineral Petrol 174, 38. https://doi.org/10.1007/s00410-019-1565-7

Timms, N.E., Reddy, S.M., Healy, D., Nemchin, A.A., Grange, M.L., Pidgeon, R.T., Hart, R., 2012. Resolution of impact-related microstructures in lunar zircon: A shockdeformation mechanism map. Meteoritics \& Planetary Science 47, 120-141. https://doi.org/10.1111/j.1945-5100.2011.01316.x

Trepmann, C.A., 2008. Shock effects in quartz: Compression versus shear deformation - An example from the Rochechouart impact structure, France. Earth and Planetary Science Letters 267, 322-332. https://doi.org/10.1016/j.epsl.2007.11.035

Trepmann, C.A., Spray, J.G., 2006. Shock-induced crystal-plastic deformation and postshock annealing of quartz: Microstructural evidence from crystalline target rocks of the Charlevoix impact structure, Canada. European Journal of Mineralogy 18, 161173. https://doi.org/10.1127/0935-1221/2006/0018-0161

Wells, A.T., Forman, D.J., Ranford, L.C., Cook, P.J., 1970. Geology of the Amadeus Basin, central Australia. Bulletin of the Bureau of Mineral Resources, Geology, and Geophysics 100, 282.

Wieland, F., Reimold, W.U., Gibson, R.L., 2006. New observations on shatter cones in the Vredefort impact structure, South Africa, and evaluation of current hypotheses for shatter cone formation. Meteoritics \& Planetary Science 41, 1737-1759. https://doi.org/10.1111/j.1945-5100.2006.tb00449.x

Wilk, J., Kenkmann, T., 2016. Formation of shatter cones in MEMIN impact experiments. Meteoritics \& Planetary Science 51, 1477-1496. https://doi.org/10.1111/maps.12682

Wünnemann, K., Collins, G.S., Melosh, H.J., 2006. A strain-based porosity model for use in hydrocode simulations of impacts and implications for transient crater growth in porous targets. Icarus 180, 514-527. https://doi.org/10.1016/j.icarus.2005.10.013 
This is a post-print of an article published in ICARUS (doi: 10.1016/j.icarus.2021.114687).

\title{
Stress and Strain during Shock Metamorphism - Supplementary Material
}

\author{
Auriol S. P. Rae ${ }^{1,2^{*}}$, Michael H. Poelchau ${ }^{1}$, and Thomas Kenkmann ${ }^{1}$ \\ ${ }^{1}$ Institute of Earth and Environmental Sciences - Geology, Albert-Ludwigs Universität \\ Freiburg, Albertstraße 23b, 79104 Freiburg, Germany. \\ ${ }^{2}$ Department of Earth Sciences, University of Cambridge, Cambridge CB2 3EQ, UK. \\ *Corresponding author email: auriol.rae@geologie.uni-freiburg.de
}

\section{Introduction}

This supplementary material is composed of five parts:

First, in this study we have exclusively used the jet-penetration formula to estimate the point source of an impact. For completeness, and to provide some consideration of the uncertainties in our analysis that result from the use of the jet-penetration formula, we present a comparison of the jet-penetration formula and $\pi$-group scaling as methods to estimate the equivalent depth of burst in Figure S1.

Second, in Figures S2 and S3 we demonstrate figures equivalent to Figure 3a and 5a where instead of comparing the orientations of principal stress and instantaneous strain between peak pressure and release, we compare those orientations between initially reaching the HEL during pressure rise (i.e. before peak pressure - PP) and peak pressure. Figure S2 demonstrates that the orientation of stress remains generally constant throughout the rise phase, the greatest exceptions to this arise along near-horizontal transects, close to the point of impact. The average deviation between these orientations is $4.2 \pm 3.8^{\circ}$.

Third, in Figures S4-7 we present profiles of deviatoric stress and instantaneous strain for the simulation presented in the main body of this study $\left(\mathrm{L}=1 \mathrm{~km}, \mathrm{vi}_{\mathrm{i}}=15 \mathrm{~km} / \mathrm{s}\right)$. 
This is a post-print of an article published in ICARUS (doi: 10.1016/j.icarus.2021.114687).

We have chosen to show profiles along the $40^{\circ}$ and $80^{\circ}$ transects to complement the profiles along $0^{\circ}$ (horizontal) shown in Figures 4 and 7.

Fourth, while the main body of this text focusses on one simulation to demonstrate how stress and strain vary by location in the target during shock metamorphism, in Figures S8-35 we present the equivalent results for four simulations with varying impactor diameter $(0.1,1$, and $10 \mathrm{~km})$ and impact velocity $(10,15,20 \mathrm{~km} / \mathrm{s})$ to demonstrate the general applicability of our results for all impact structures.

Finally, we have included 5 Supplementary Videos (SV1-5) that show the development of the stress ellipsoid during shock metamorphism on Mohr circle diagrams. Each Supplementary Video (1-5) corresponds, respectively, to each row of Figure 4, and as a special case, SV3 also corresponds to the same Lagrangian pseudocell as Figure 5. Each video begins at the time the HEL is first exceeded and ends at the time that pressures decrease back to the HEL. SV1 shows the state of stress in a Lagrangian pseudocell that is initially $1.0 \mathrm{r} / \mathrm{L}$ from the point source along the $\alpha=0^{\circ}$ transect. Each subsequent video shows the stress state in Lagrangian pseudocells along the same transect at increasing radial distance (increments of $0.5 \mathrm{r} / \mathrm{L}$ ). 
This is a post-print of an article published in ICARUS (doi: 10.1016/j.icarus.2021.114687).

\section{The Point Source Approximation}

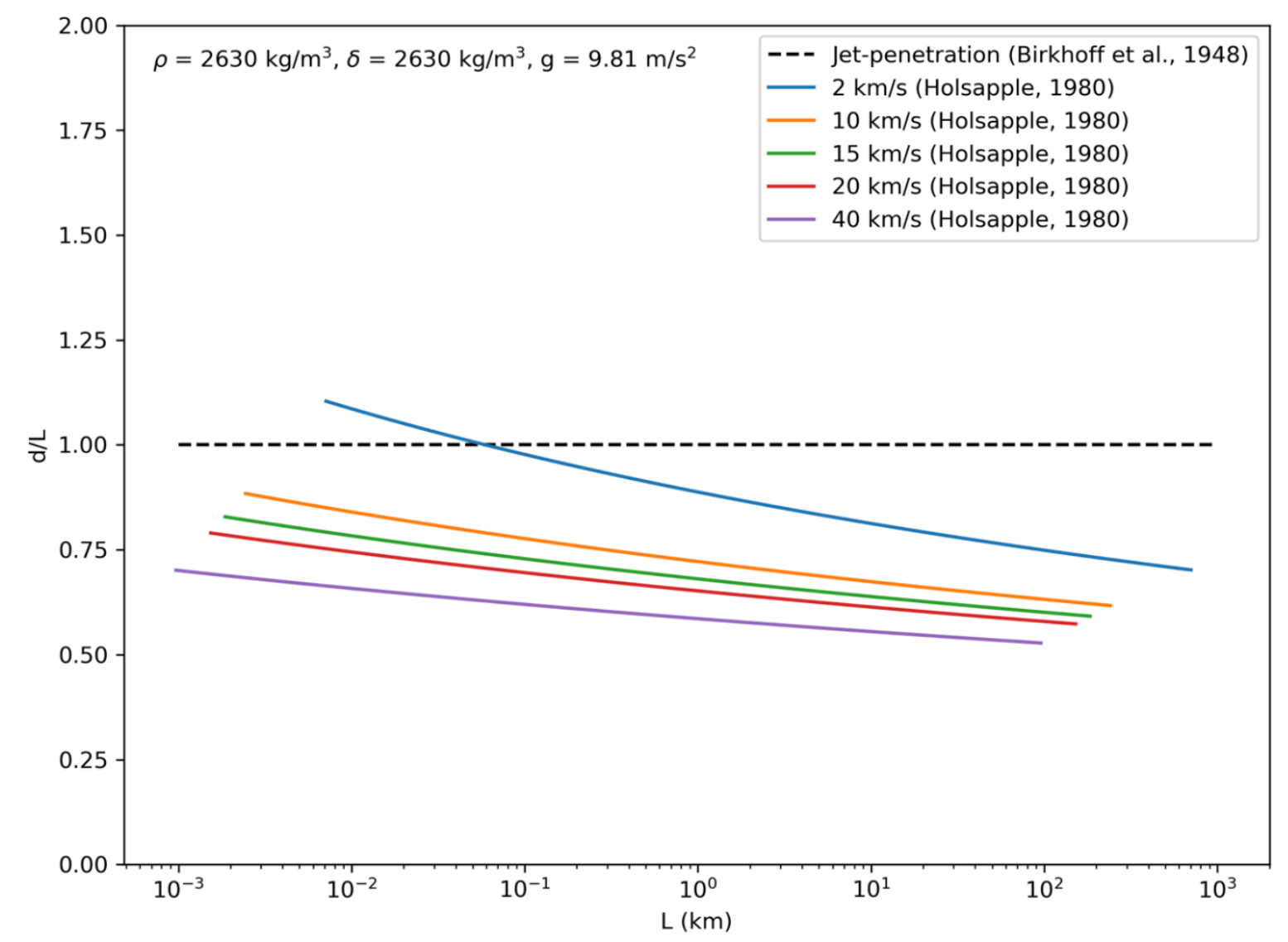

Figure S1. Equivalent depth of burst calculated by the jet-penetration formula (Birkhoff et al., 1948) compared to $\pi$-group scaling (Holsapple, 1980). The jet-penetration formula is independent impact velocity or size, while $\pi$-group scaling has some dependency over the considered impactor size range. d: depth of burst; L: impactor diameter; $\rho$ : target density; $\delta$ : impactor density. 
This is a post-print of an article published in ICARUS (doi: 10.1016/j.icarus.2021.114687).

\section{Principal Orientation Rotation between HEL and Peak Pressure}

- $\sigma_{1}$ at HEL (before PP) $\quad \sigma_{1}$ at Peak Pressure
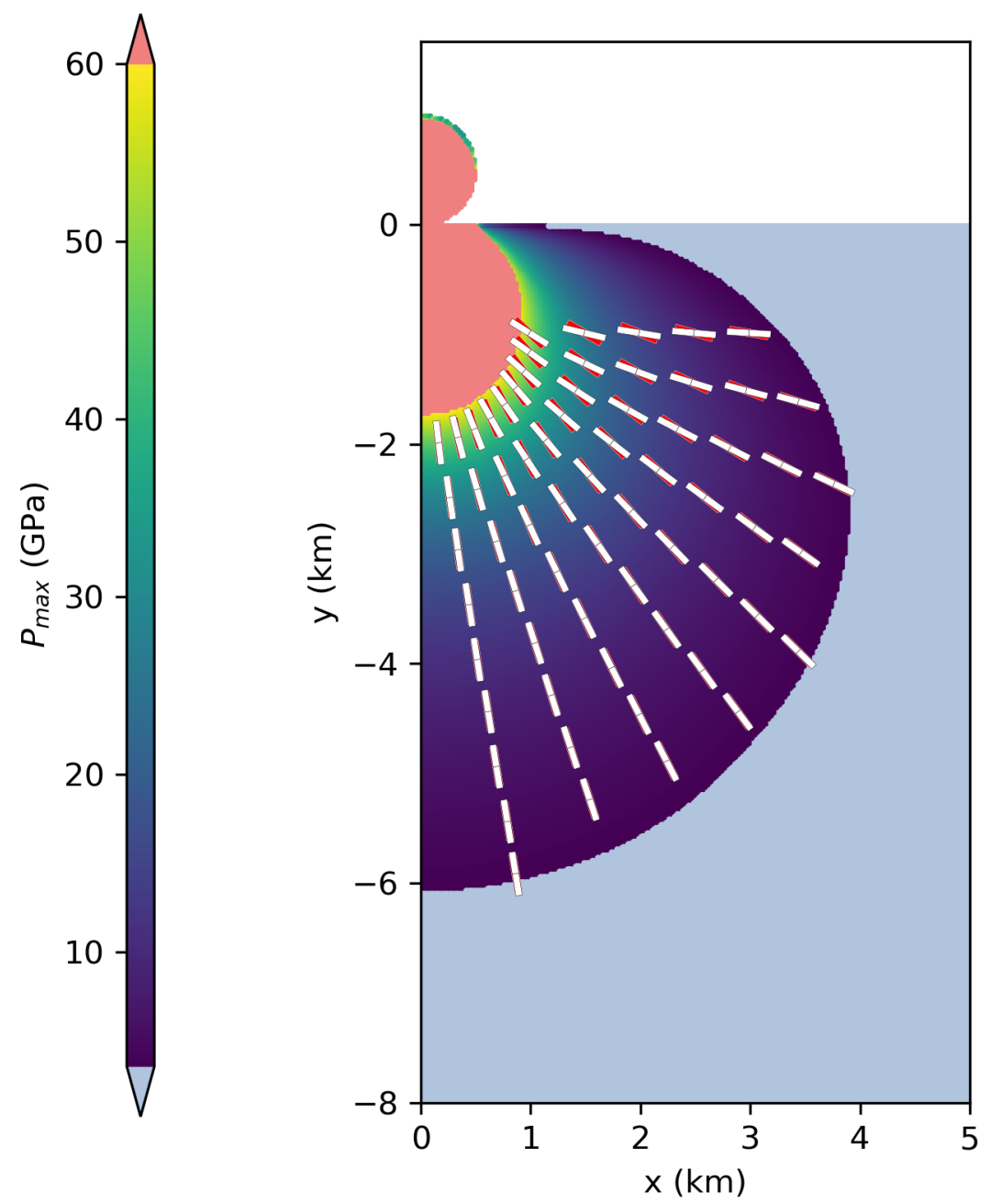

Figure 2. The orientation of principal stresses between the HEL being exceeded (before peak pressure) and peak pressure. Material is plotted at its pre-impact location and is colored by peak shock pressure. Lines indicate the orientation of maximum principal stress as the HEL is exceeded (red) and peak pressure (white). 
This is a post-print of an article published in ICARUS (doi: 10.1016/j.icarus.2021.114687).

- $I S A_{3}$ at HEL (before PP) $\square I S A_{3}$ at Peak Pressure
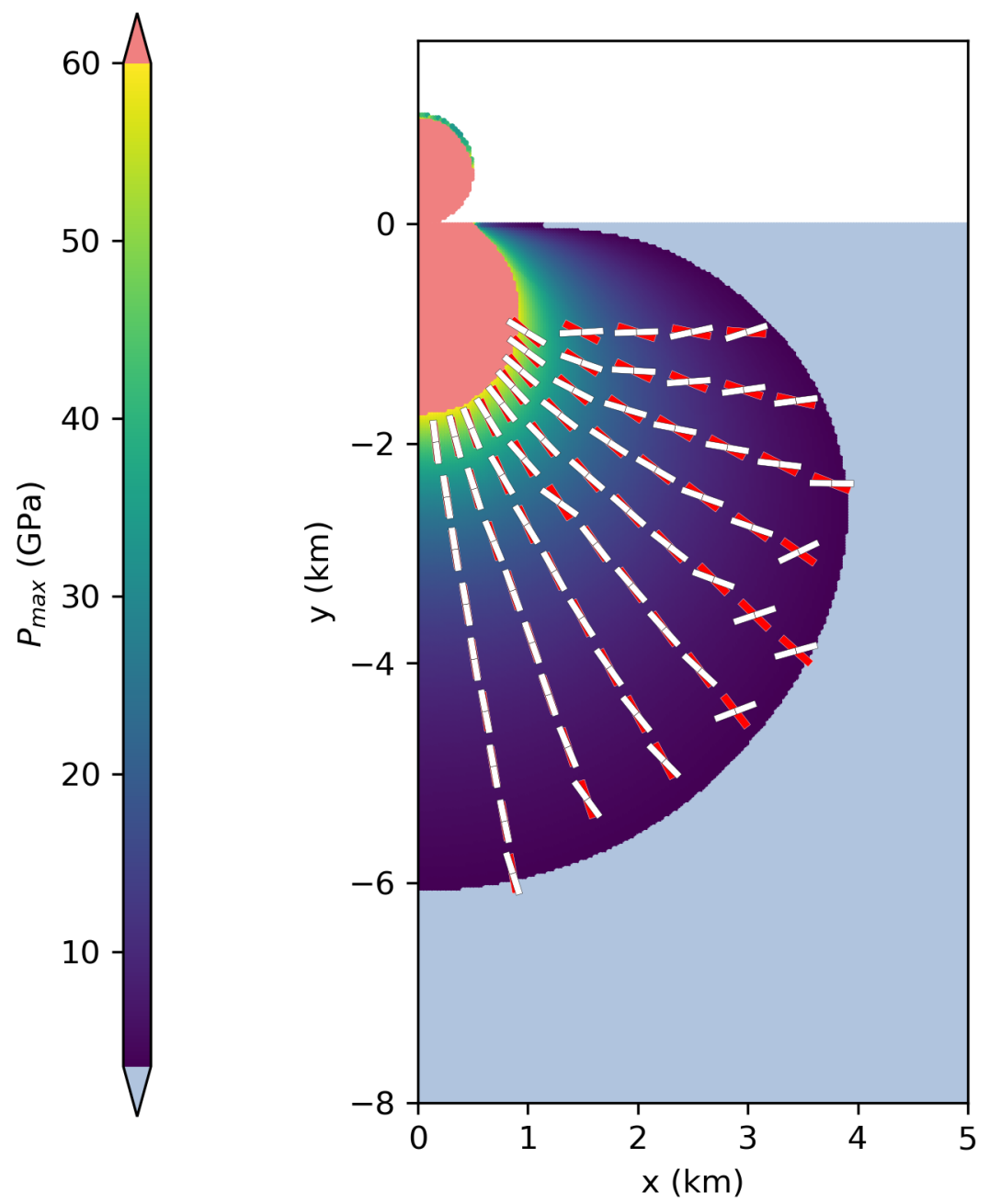

Figure 3. The orientation of instantaneous stretching axes (ISAs) between the HEL being exceeded (before peak pressure) and peak pressure. Material is plotted at its pre-impact location and is colored by peak shock pressure. Lines indicate the orientation of maximum instantaneous stretching as the HEL is exceeded (red) and peak pressure (white). 
This is a post-print of an article published in ICARUS (doi: 10.1016/j.icarus.2021.114687).

Profiles of Deviatoric Stress and Strain 
This is a post-print of an article published in ICARUS (doi: 10.1016/j.icarus.2021.114687).
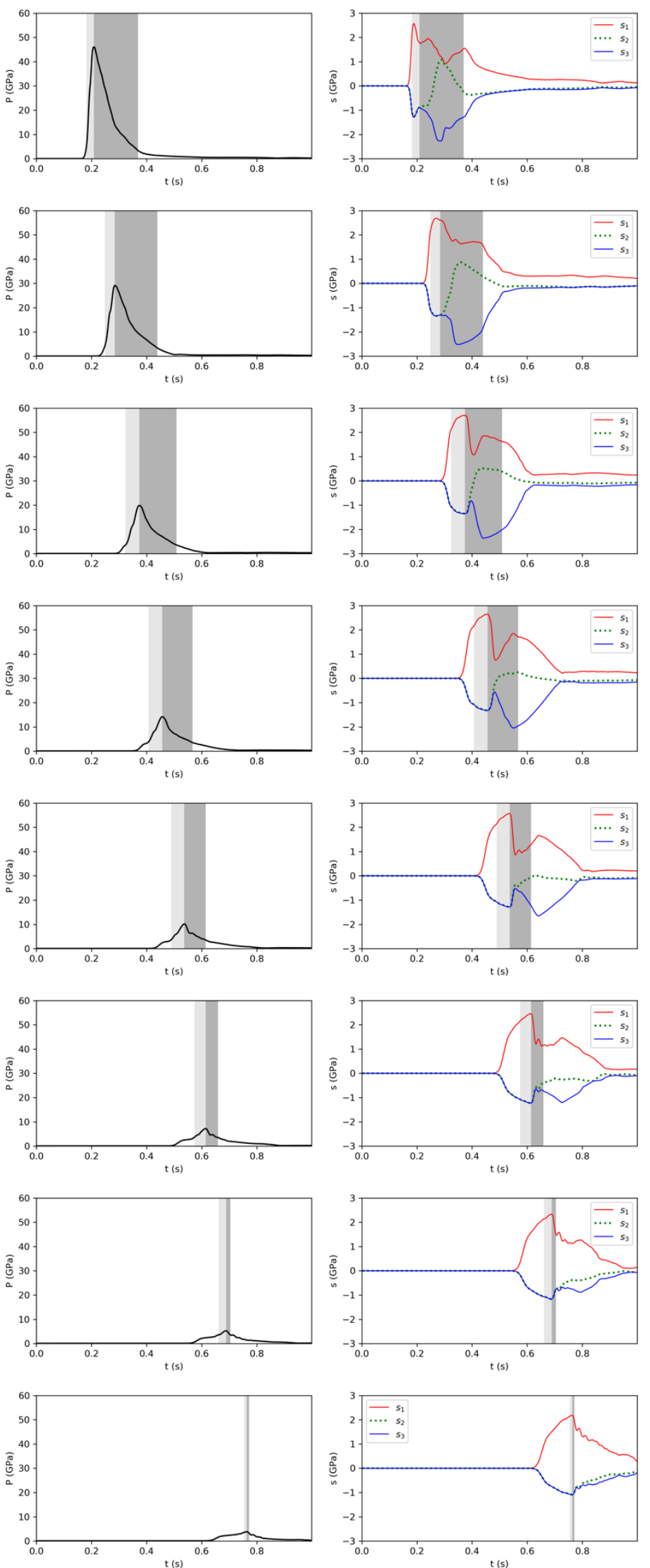

Figure 4. Stress profile along $40^{\circ}$ transect. 
This is a post-print of an article published in ICARUS (doi: 10.1016/j.icarus.2021.114687).
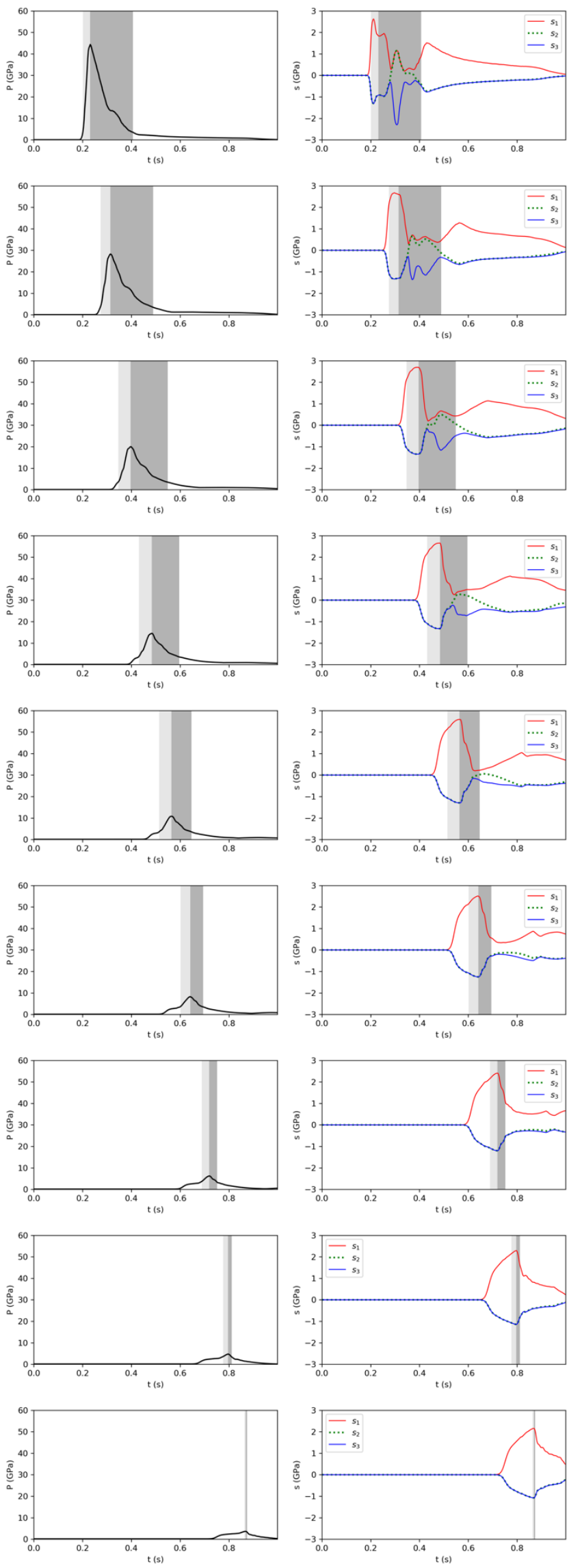

Figure 5. Stress profile along $80^{\circ}$ transect. 
This is a post-print of an article published in ICARUS (doi: 10.1016/j.icarus.2021.114687).
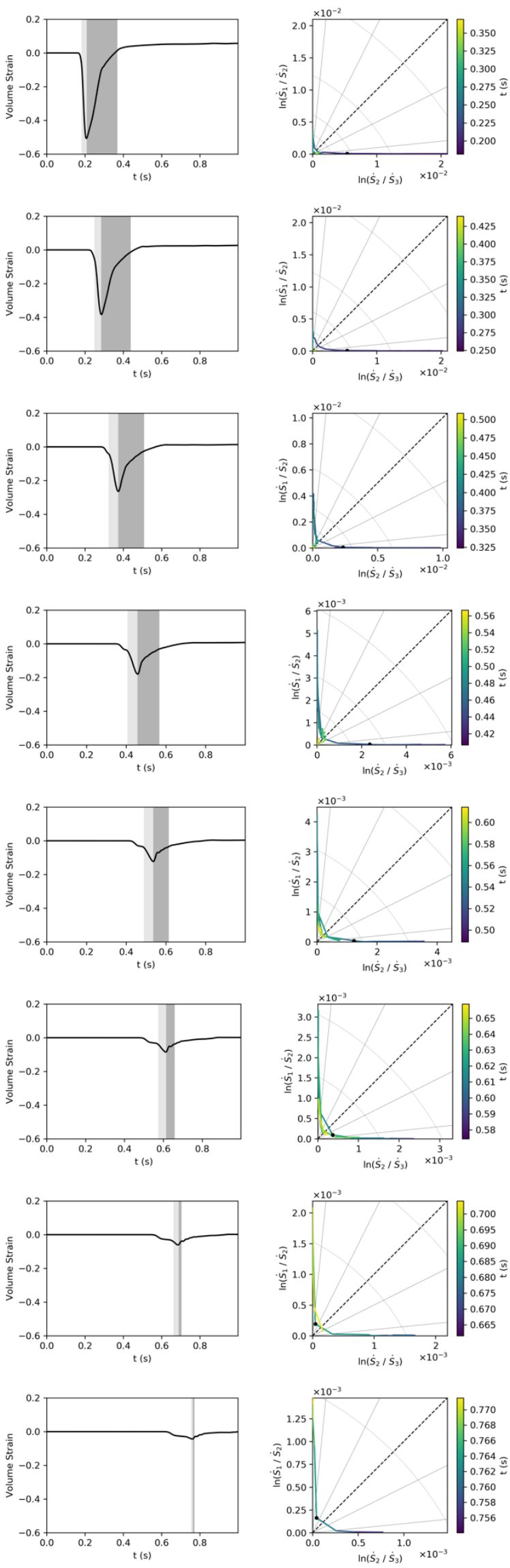

Figure 6. Strain profile along $40^{\circ}$ transect. 
This is a post-print of an article published in ICARUS (doi: 10.1016/j.icarus.2021.114687).
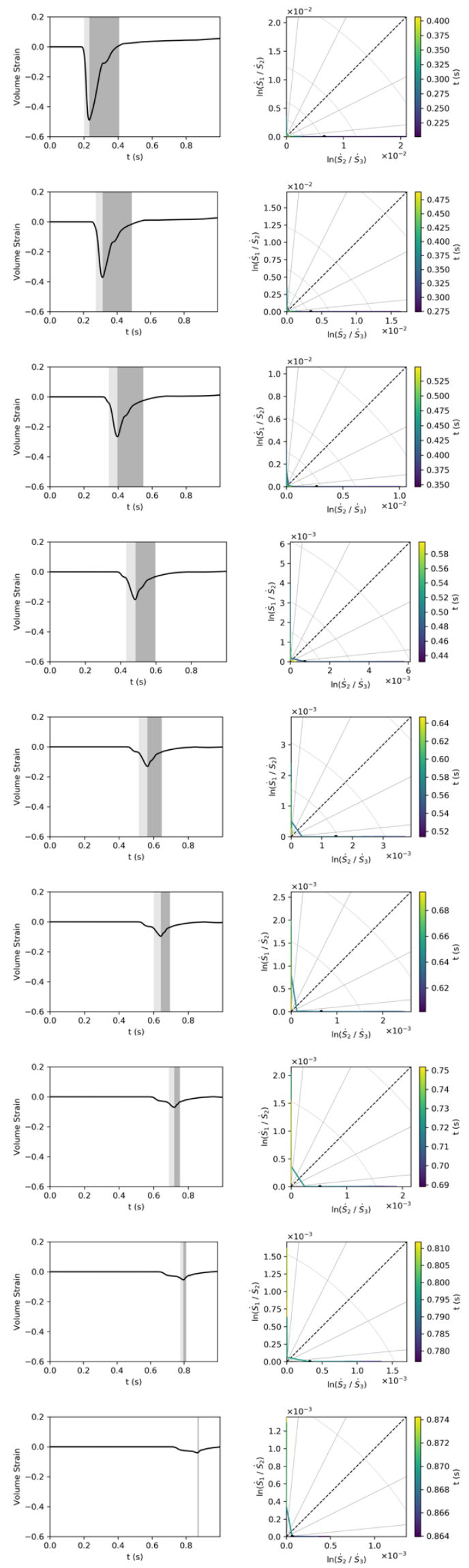

Figure 7. Strain profile along $80^{\circ}$ transect. 
This is a post-print of an article published in ICARUS (doi: 10.1016/j.icarus.2021.114687).

\section{Comparison of Results with Different Impact Parameters}

\section{Stress Orientation}

$$
\underline{\mathrm{L}}=0.1 \mathrm{~km}, \mathrm{vi}=15 \mathrm{~km} / \mathrm{s}
$$

$-\sigma_{1}$ at Peak Pressure $\quad-\sigma_{1}$ at Release
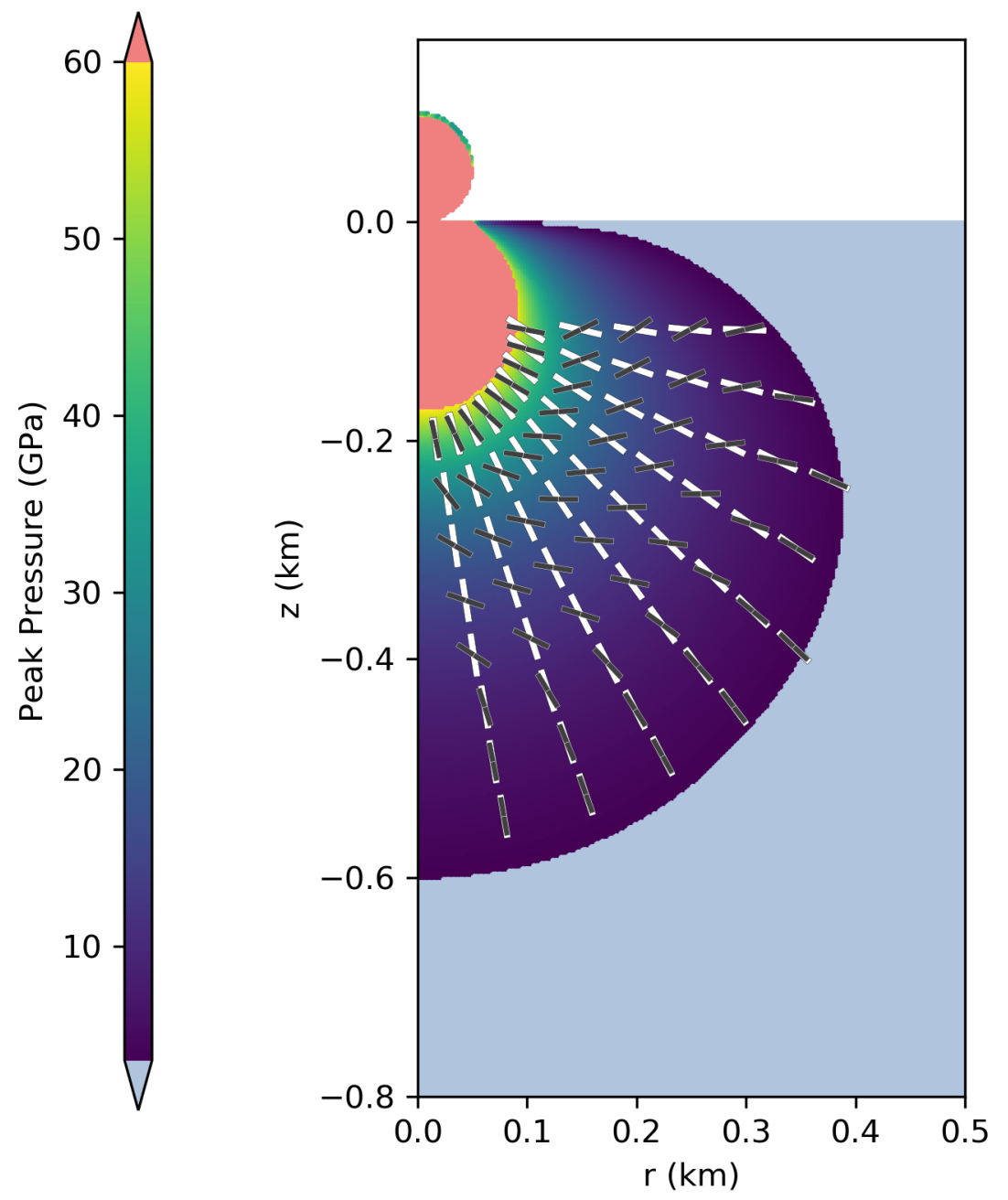

Figure 8. Stress orientations for a $0.1 \mathrm{~km}$ diameter impactor travelling at $15 \mathrm{~km} / \mathrm{s}$. 
This is a post-print of an article published in ICARUS (doi: 10.1016/j.icarus.2021.114687).
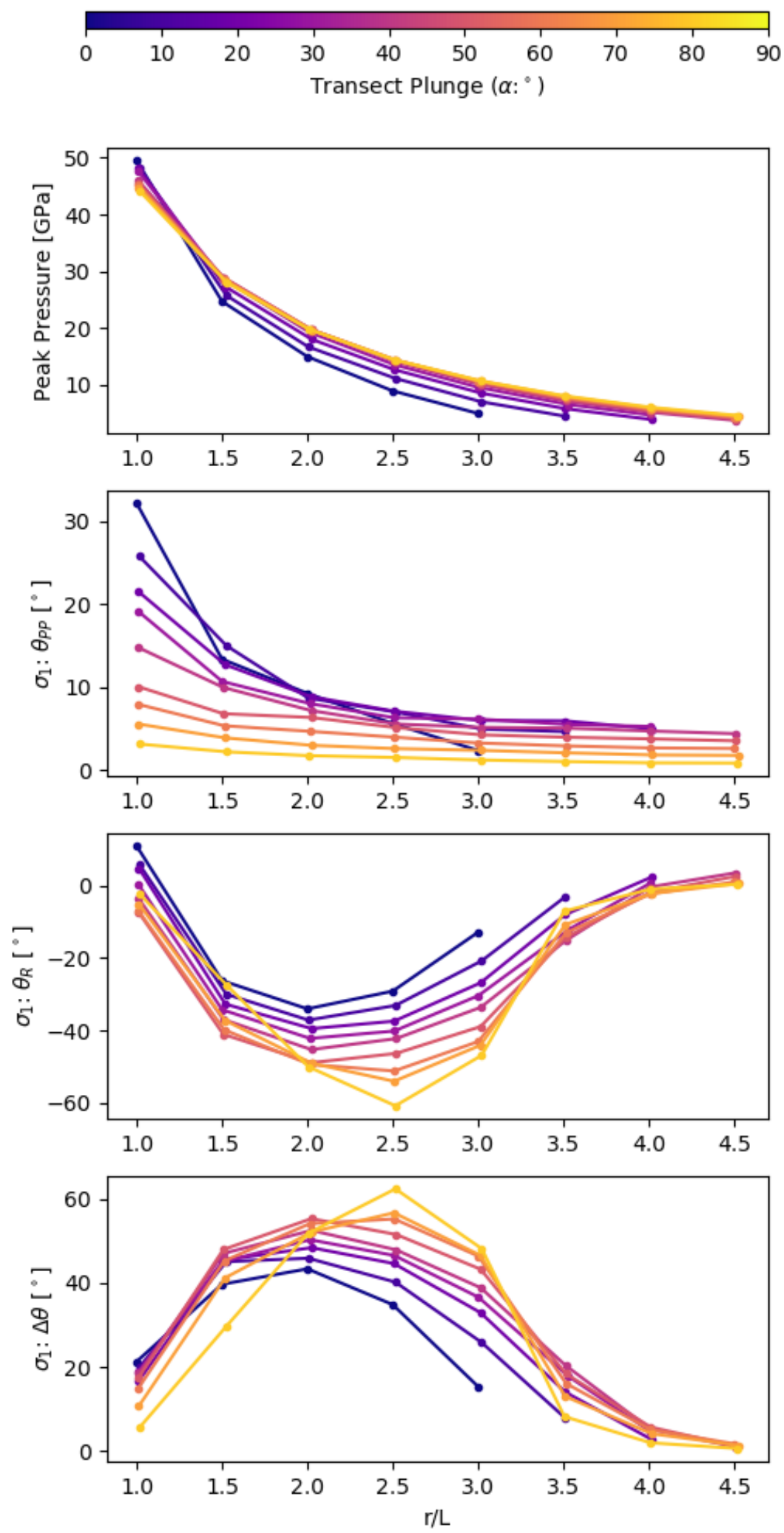

Figure 9. Stress orientations for a $0.1 \mathrm{~km}$ diameter impactor travelling at $15 \mathrm{~km} / \mathrm{s}$. 
This is a post-print of an article published in ICARUS (doi: 10.1016/j.icarus.2021.114687).

$$
\underline{\mathrm{L}}=10 \mathrm{~km}, \mathrm{vi}=15 \mathrm{~km} / \mathrm{s}
$$

$-\sigma_{1}$ at Peak Pressure $\quad-\sigma_{1}$ at Release

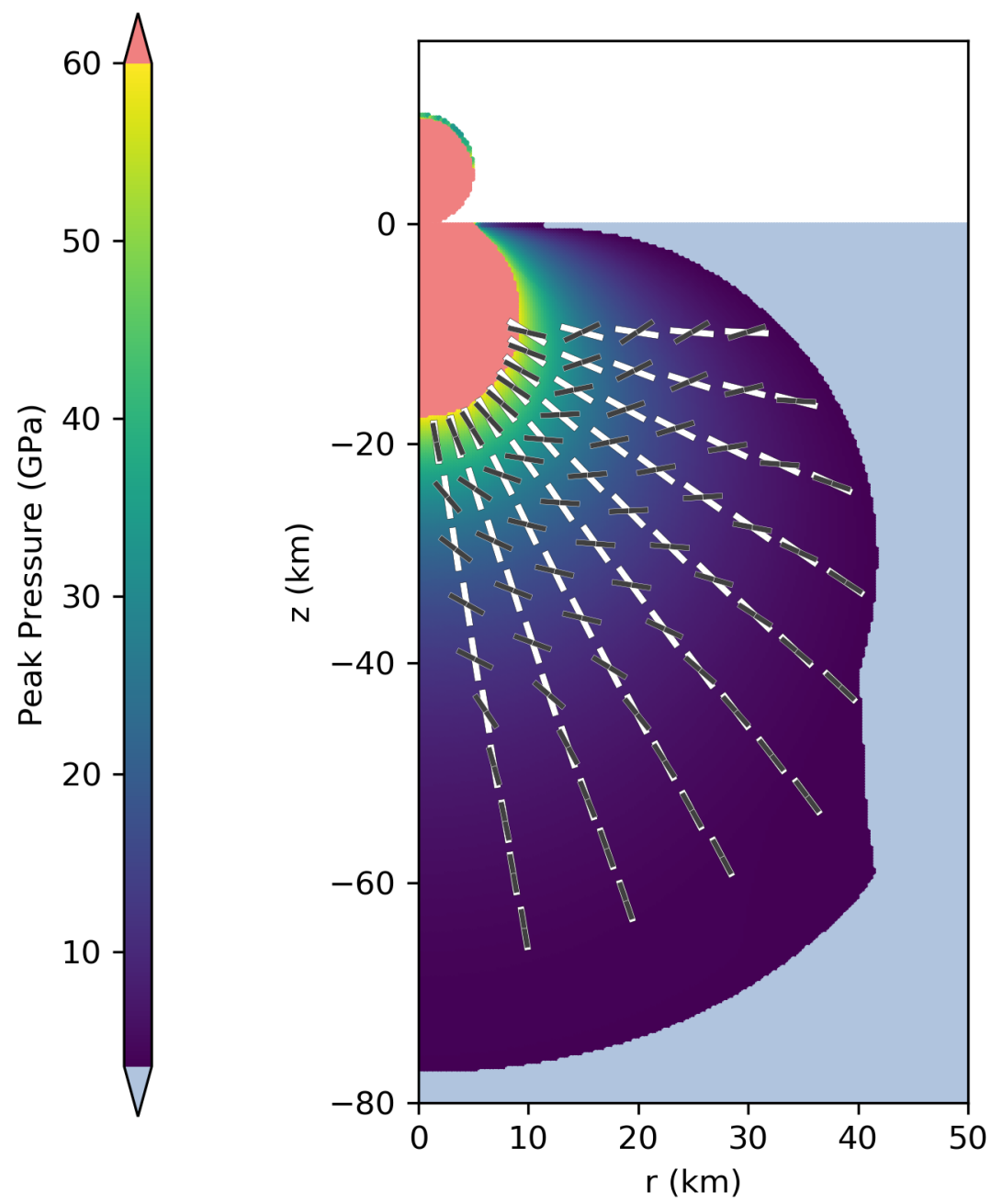

Figure 10. Stress orientations for a $10 \mathrm{~km}$ diameter impactor travelling at $15 \mathrm{~km} / \mathrm{s}$. 
This is a post-print of an article published in ICARUS (doi: 10.1016/j.icarus.2021.114687).
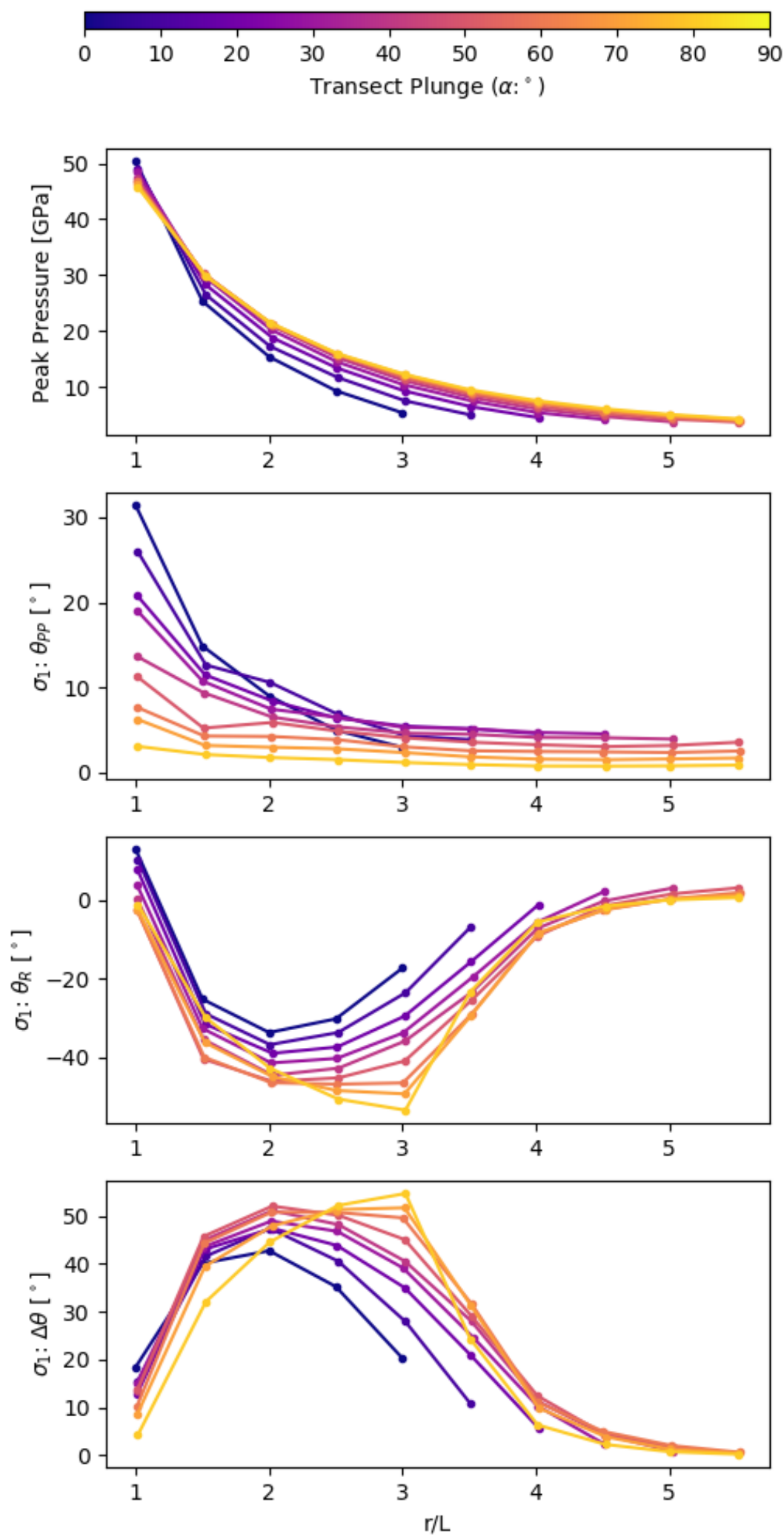

Figure 11. Stress orientations for a $10 \mathrm{~km}$ diameter impactor travelling at $15 \mathrm{~km} / \mathrm{s}$. 
This is a post-print of an article published in ICARUS (doi: 10.1016/j.icarus.2021.114687).

$$
\underline{\mathrm{L}=1 \mathrm{~km}, \mathrm{vi}=10 \mathrm{~km} / \mathrm{s}}
$$

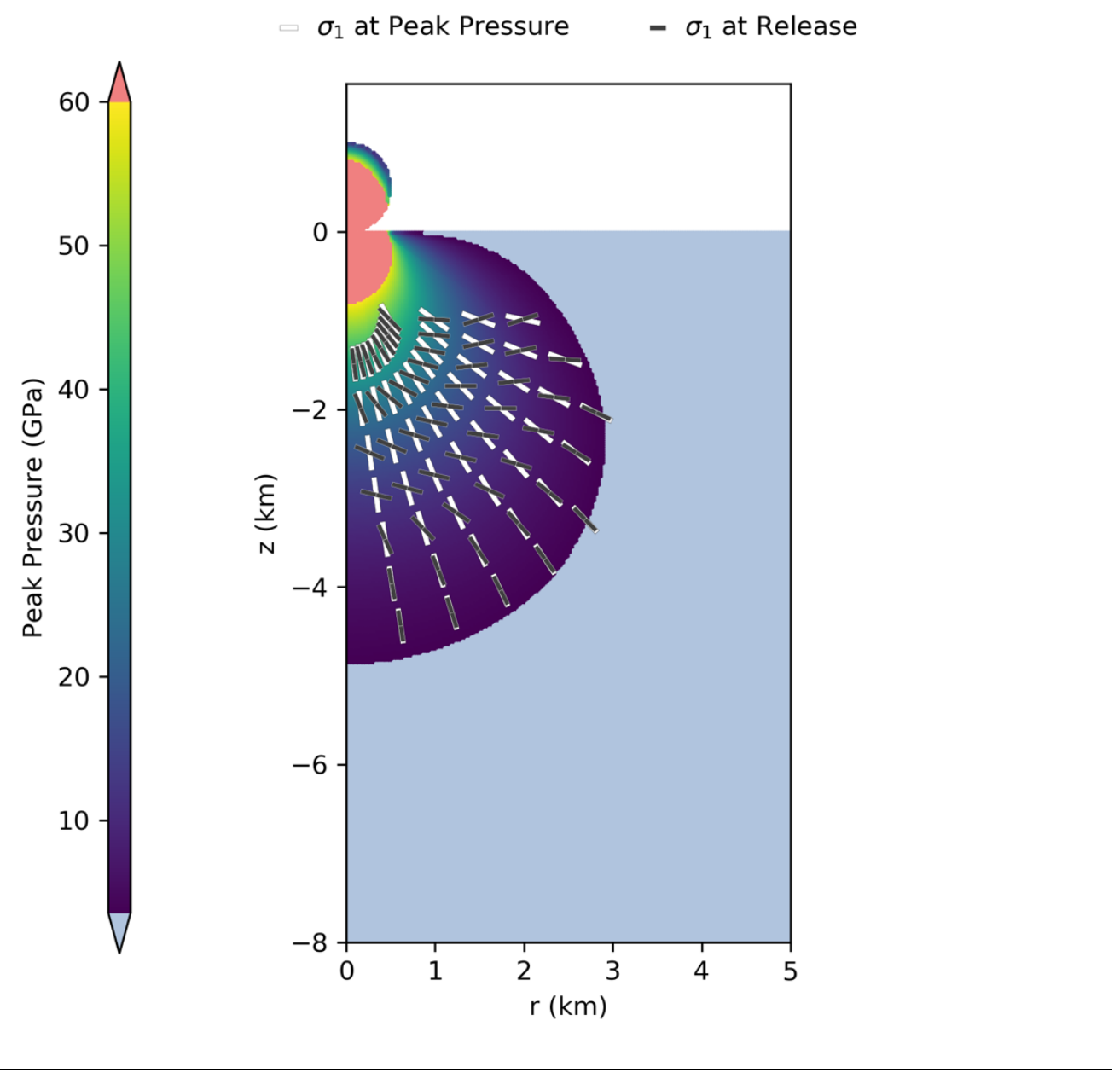

Figure 12. Stress orientations for a $1 \mathrm{~km}$ diameter impactor travelling at $10 \mathrm{~km} / \mathrm{s}$. 
This is a post-print of an article published in ICARUS (doi: 10.1016/j.icarus.2021.114687).
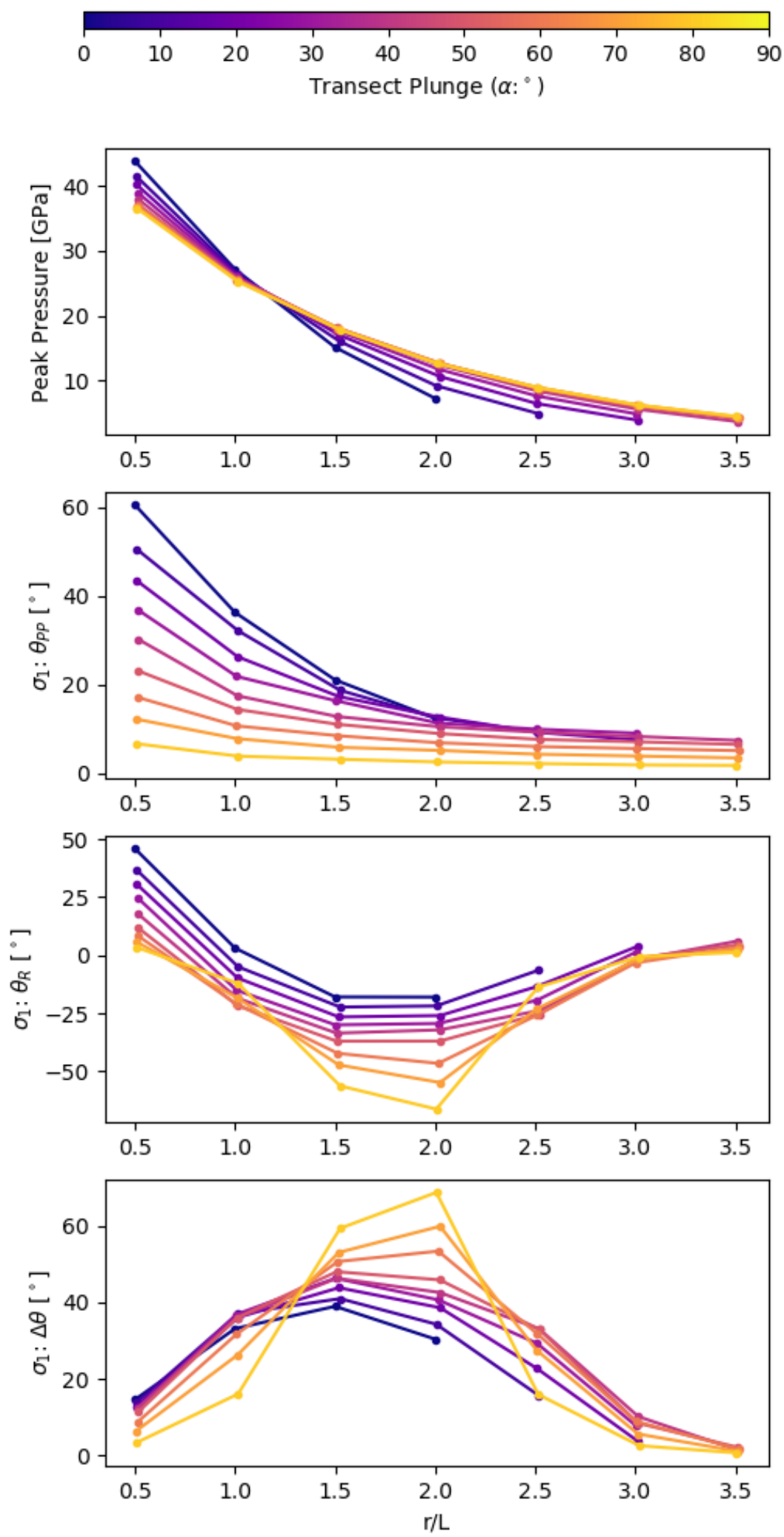

Figure 13. Stress orientations for a $1 \mathrm{~km}$ diameter impactor travelling at $10 \mathrm{~km} / \mathrm{s}$. 
This is a post-print of an article published in ICARUS (doi: 10.1016/j.icarus.2021.114687).

$$
\underline{\mathrm{L}}=1 \mathrm{~km}, \mathrm{vi}=20 \mathrm{~km} / \mathrm{s}
$$

$-\sigma_{1}$ at Peak Pressure $\quad-\sigma_{1}$ at Release

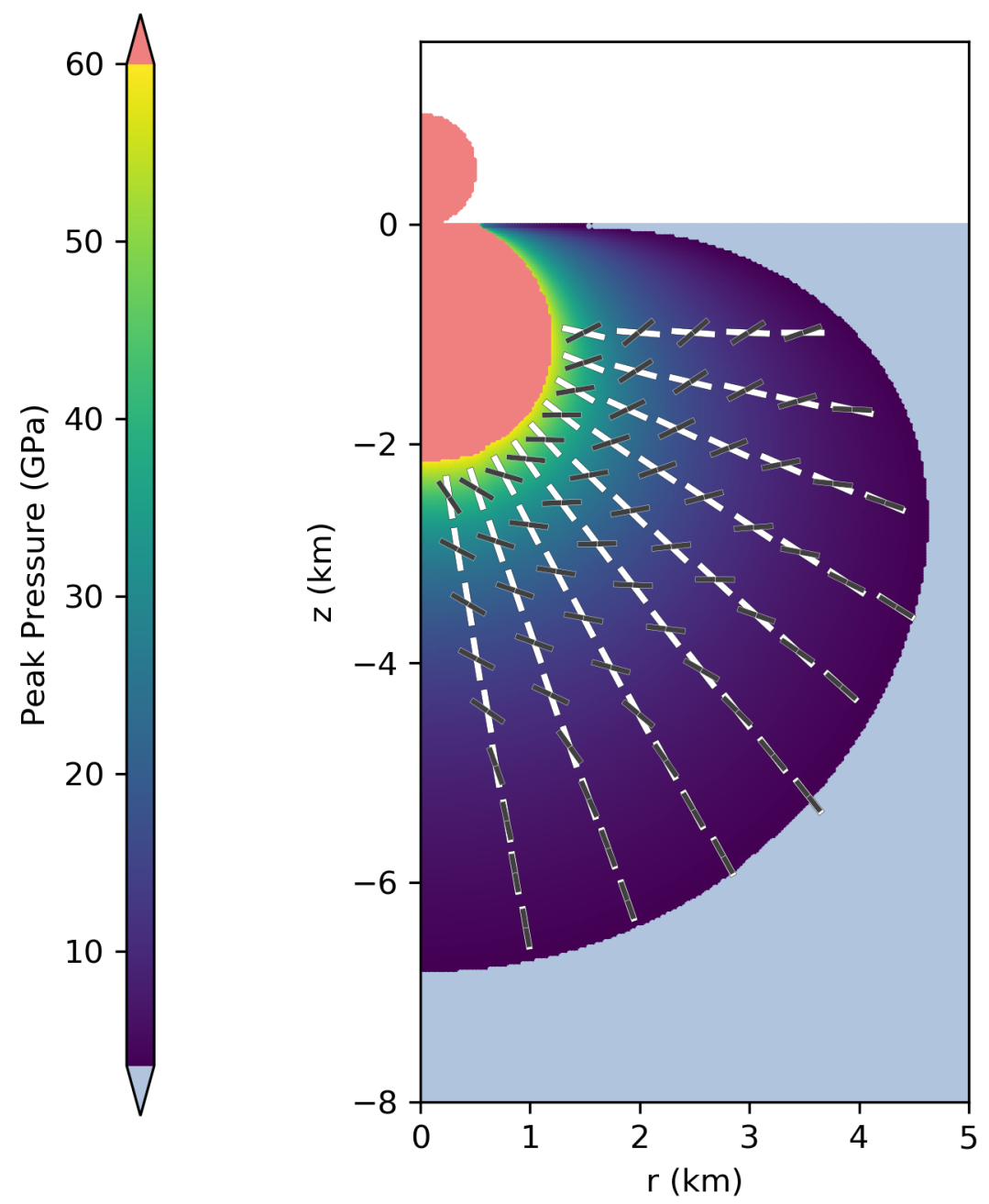

Figure 14. Stress orientations for a $1 \mathrm{~km}$ diameter impactor travelling at $20 \mathrm{~km} / \mathrm{s}$. 
This is a post-print of an article published in ICARUS (doi: 10.1016/j.icarus.2021.114687).
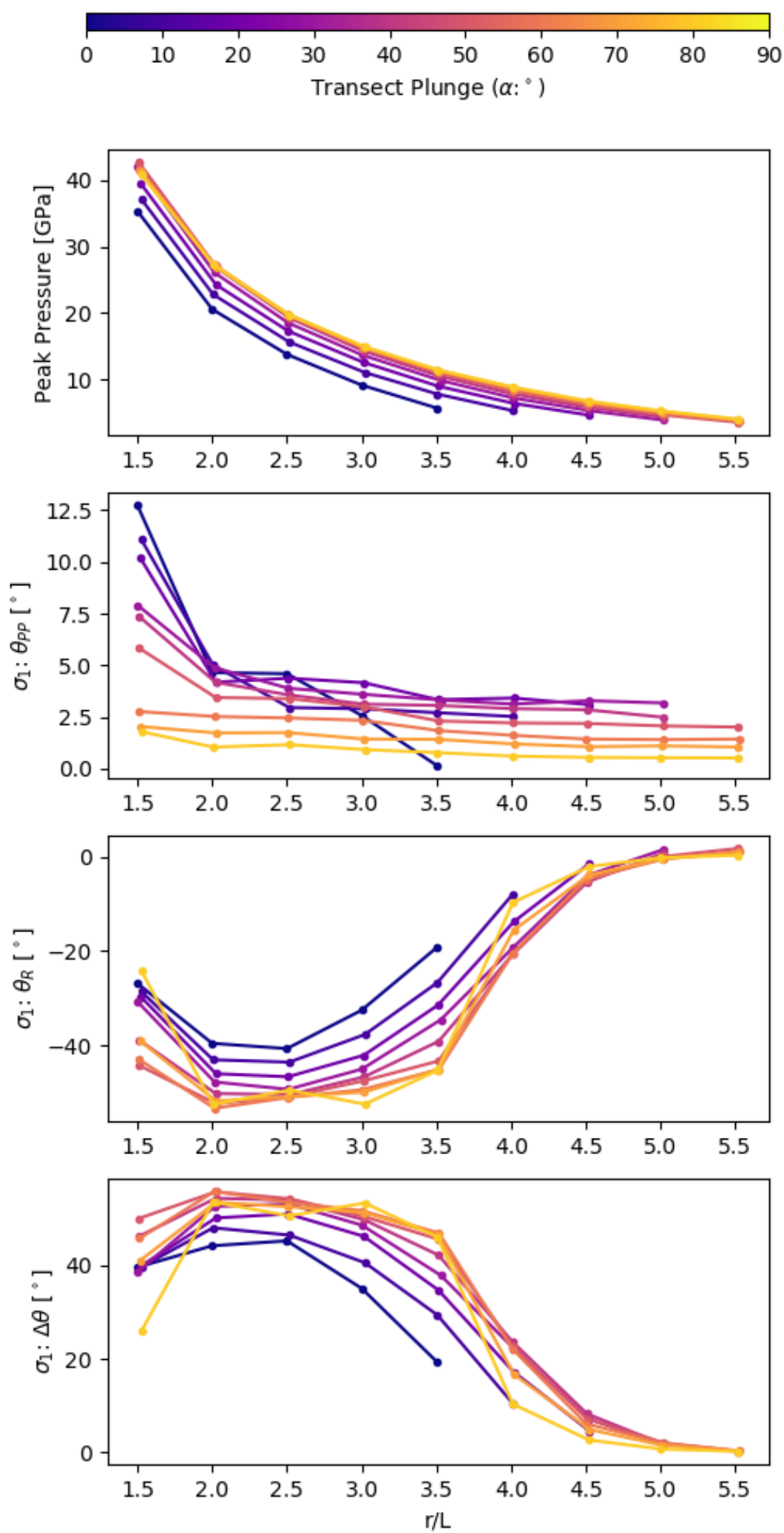

Figure 15. Stress orientations for a $1 \mathrm{~km}$ diameter impactor travelling at $20 \mathrm{~km} / \mathrm{s}$. 
This is a post-print of an article published in ICARUS (doi: 10.1016/j.icarus.2021.114687).

\section{Instantaneous Strain Orientation}

$$
\underline{\mathrm{L}}=0.1 \mathrm{~km}, \mathrm{vi}=15 \mathrm{~km} / \mathrm{s}
$$
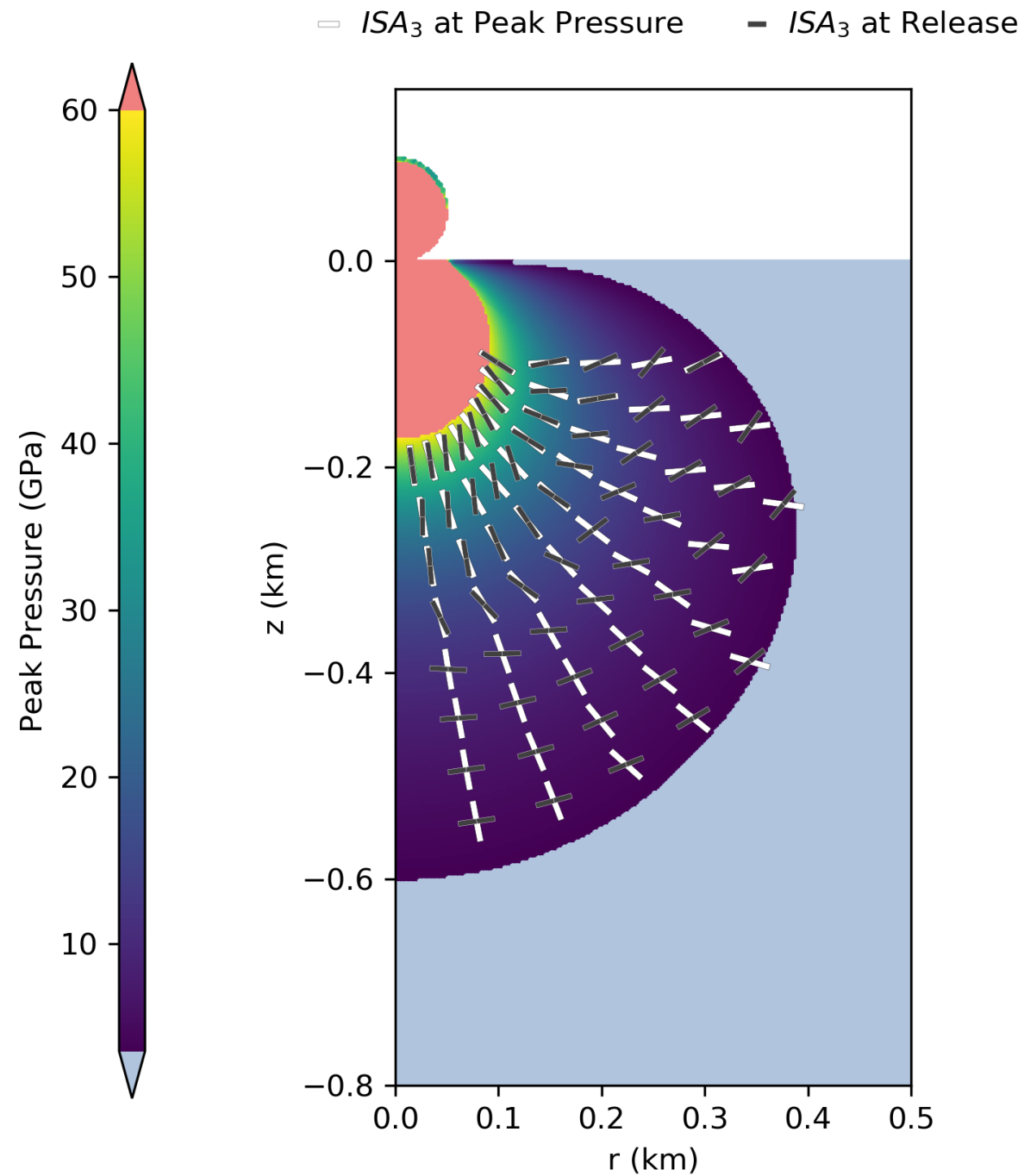

Figure 16. Instantaneous strain orientations for a $0.1 \mathrm{~km}$ diameter impactor travelling at 15 $\mathrm{km} / \mathrm{s}$. 
This is a post-print of an article published in ICARUS (doi: 10.1016/j.icarus.2021.114687).
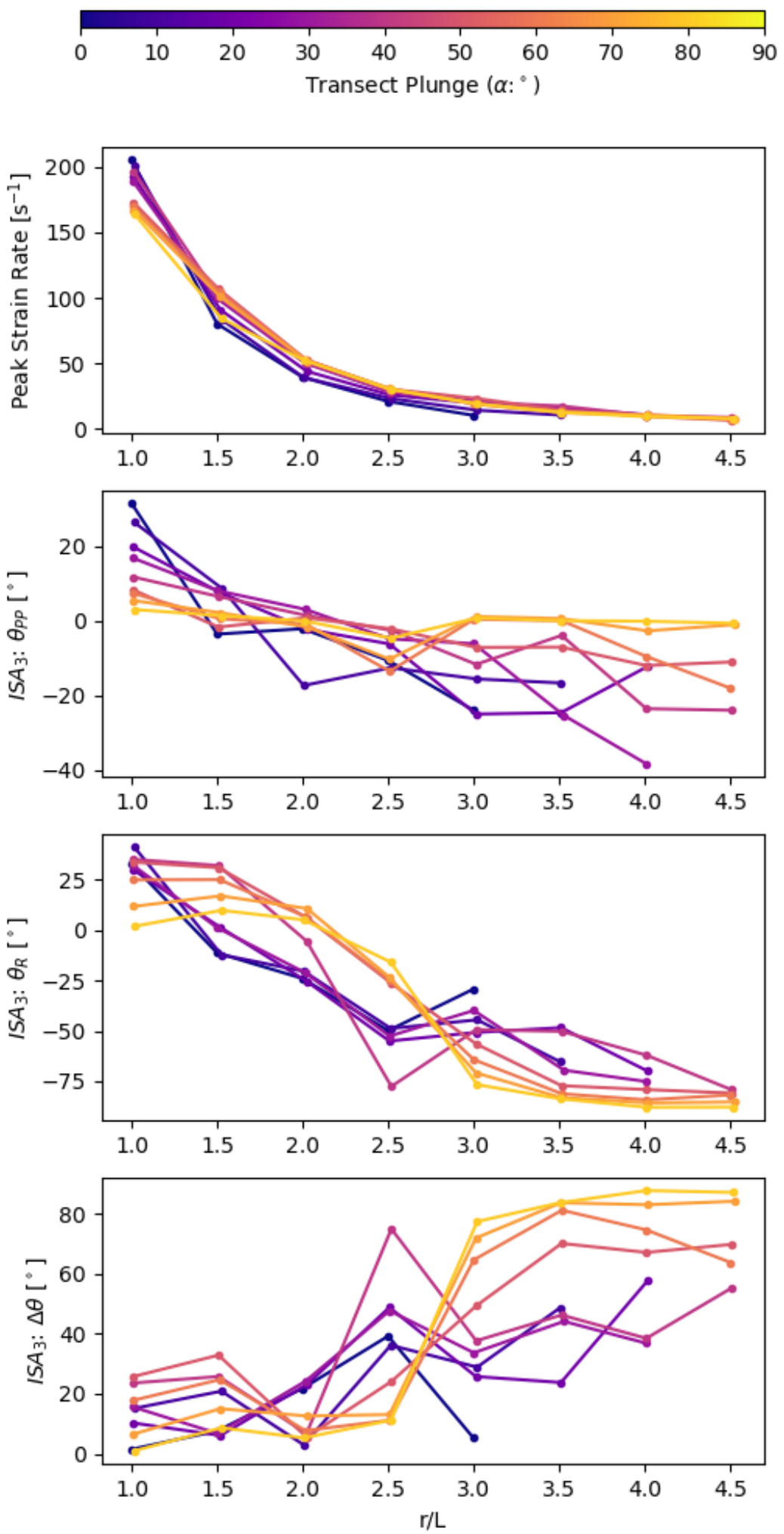

Figure 17. Instantaneous strain orientations for a $0.1 \mathrm{~km}$ diameter impactor travelling at 15 $\mathrm{km} / \mathrm{s}$. 
This is a post-print of an article published in ICARUS (doi: 10.1016/j.icarus.2021.114687).

$$
\underline{\mathrm{L}}=10 \mathrm{~km}, \mathrm{vi}=15 \mathrm{~km} / \mathrm{s}
$$

$-I S A_{3}$ at Peak Pressure $\quad-I S A_{3}$ at Release

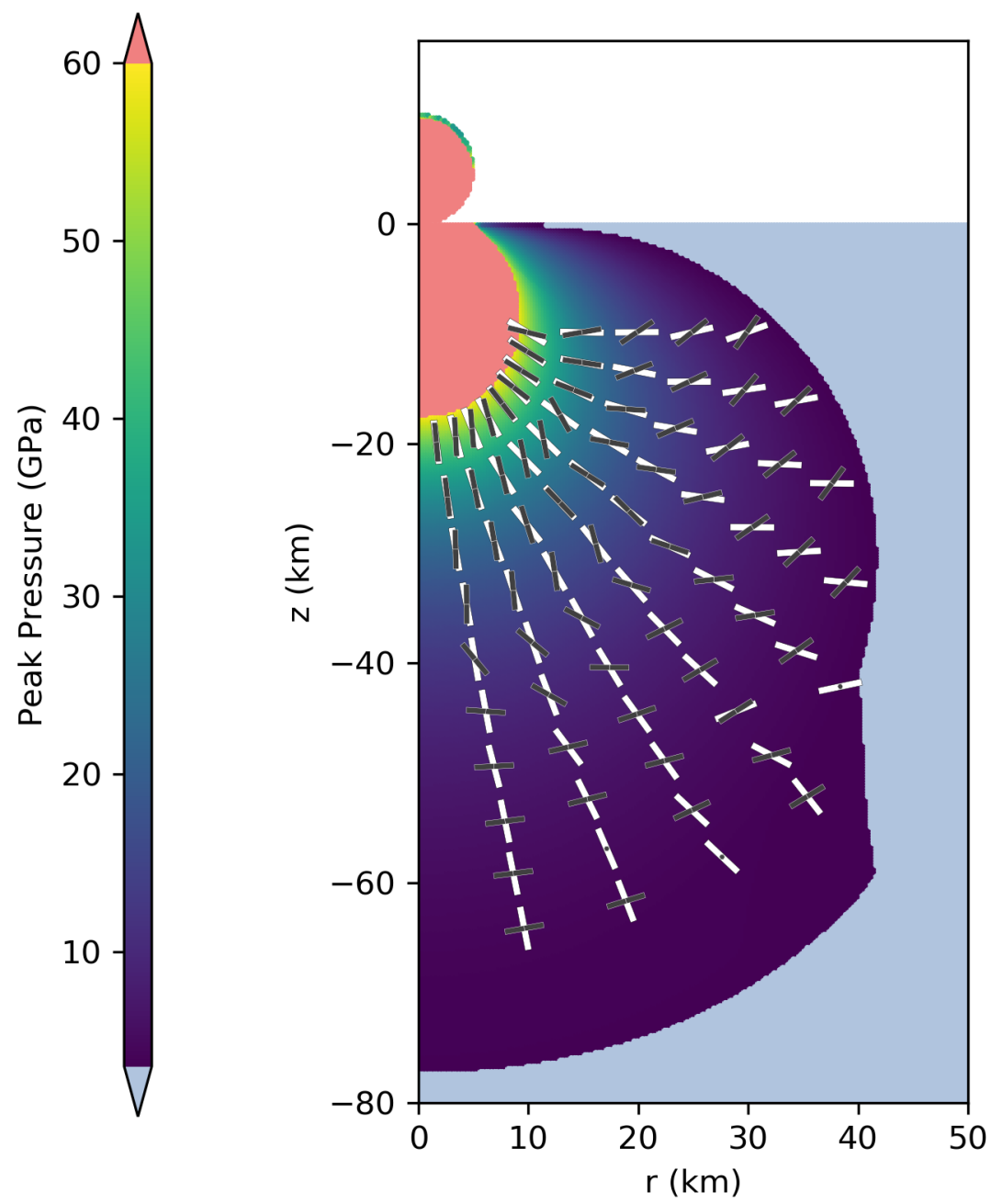

Figure 18. Instantaneous strain orientations for a $10 \mathrm{~km}$ diameter impactor travelling at 15 $\mathrm{km} / \mathrm{s}$. 
This is a post-print of an article published in ICARUS (doi: 10.1016/j.icarus.2021.114687).
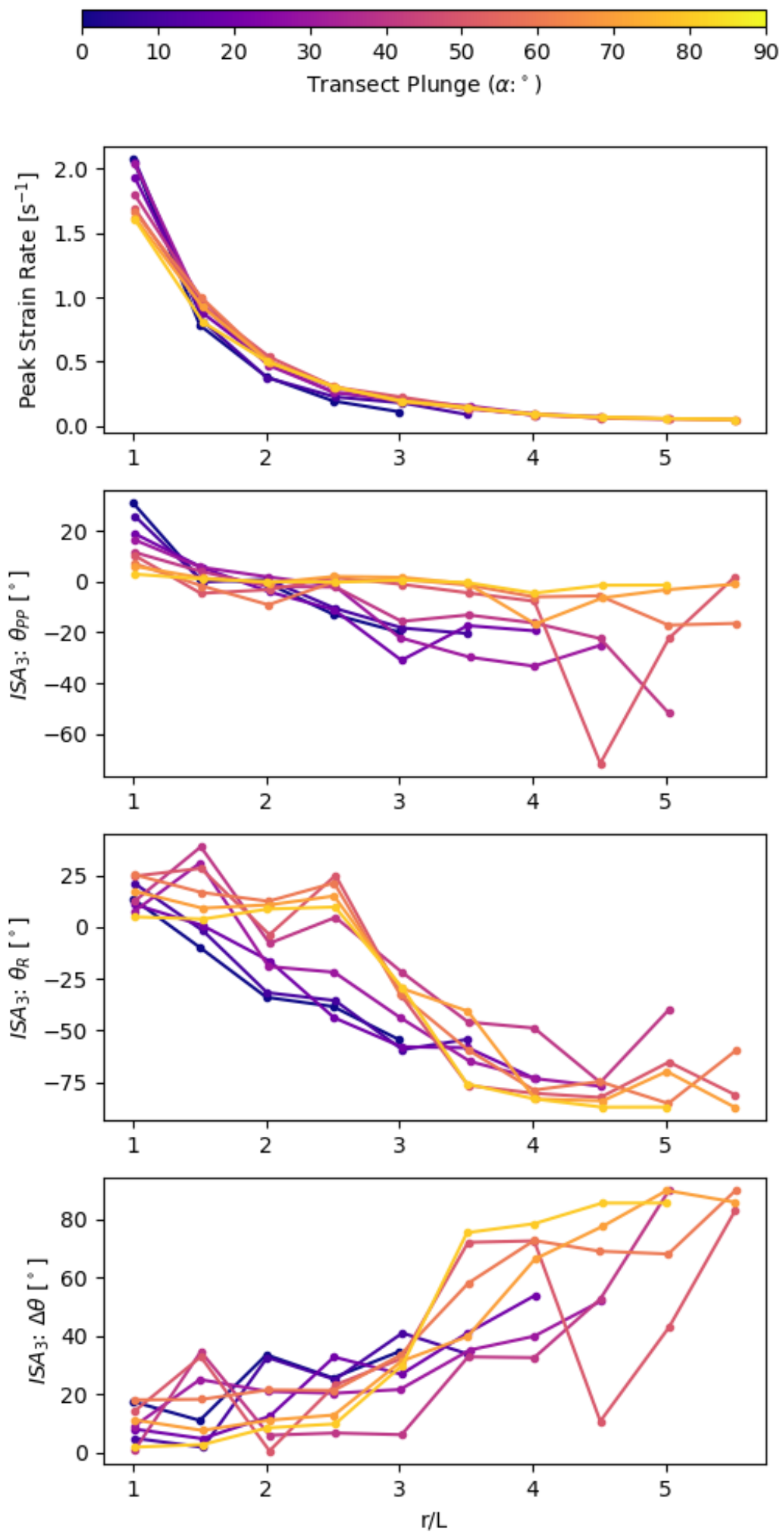

Figure 19. Instantaneous strain orientations for a $10 \mathrm{~km}$ diameter impactor travelling at 15 $\mathrm{km} / \mathrm{s}$. 
This is a post-print of an article published in ICARUS (doi: 10.1016/j.icarus.2021.114687).

$$
\underline{\mathrm{L}=1 \mathrm{~km}, \mathrm{vi}=10 \mathrm{~km} / \mathrm{s}}
$$

- $I S A_{3}$ at Peak Pressure $\quad-I S A_{3}$ at Release
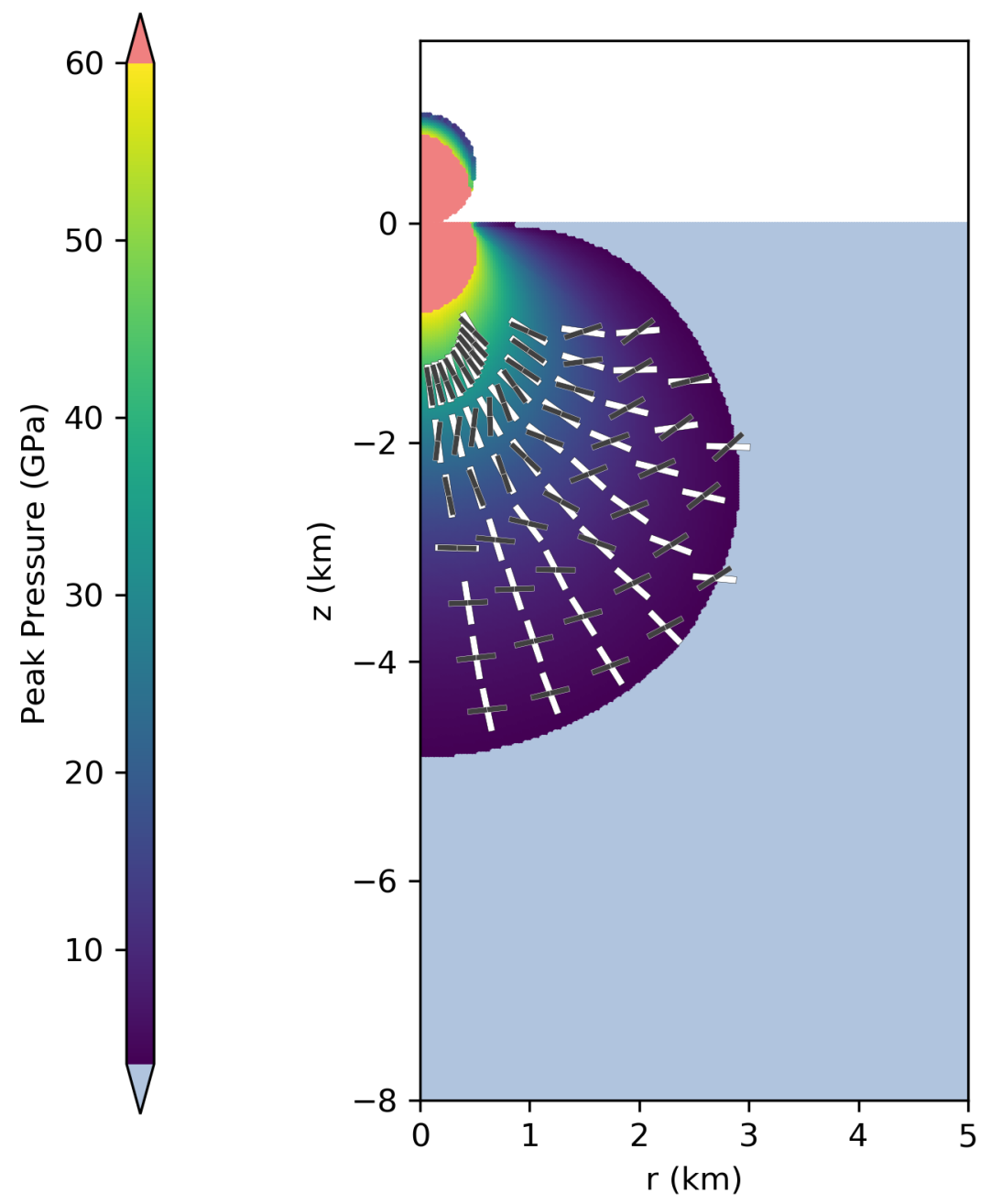

Figure 20. Instantaneous strain orientations for a $1 \mathrm{~km}$ diameter impactor travelling at 10 $\mathrm{km} / \mathrm{s}$. 
This is a post-print of an article published in ICARUS (doi: 10.1016/j.icarus.2021.114687).
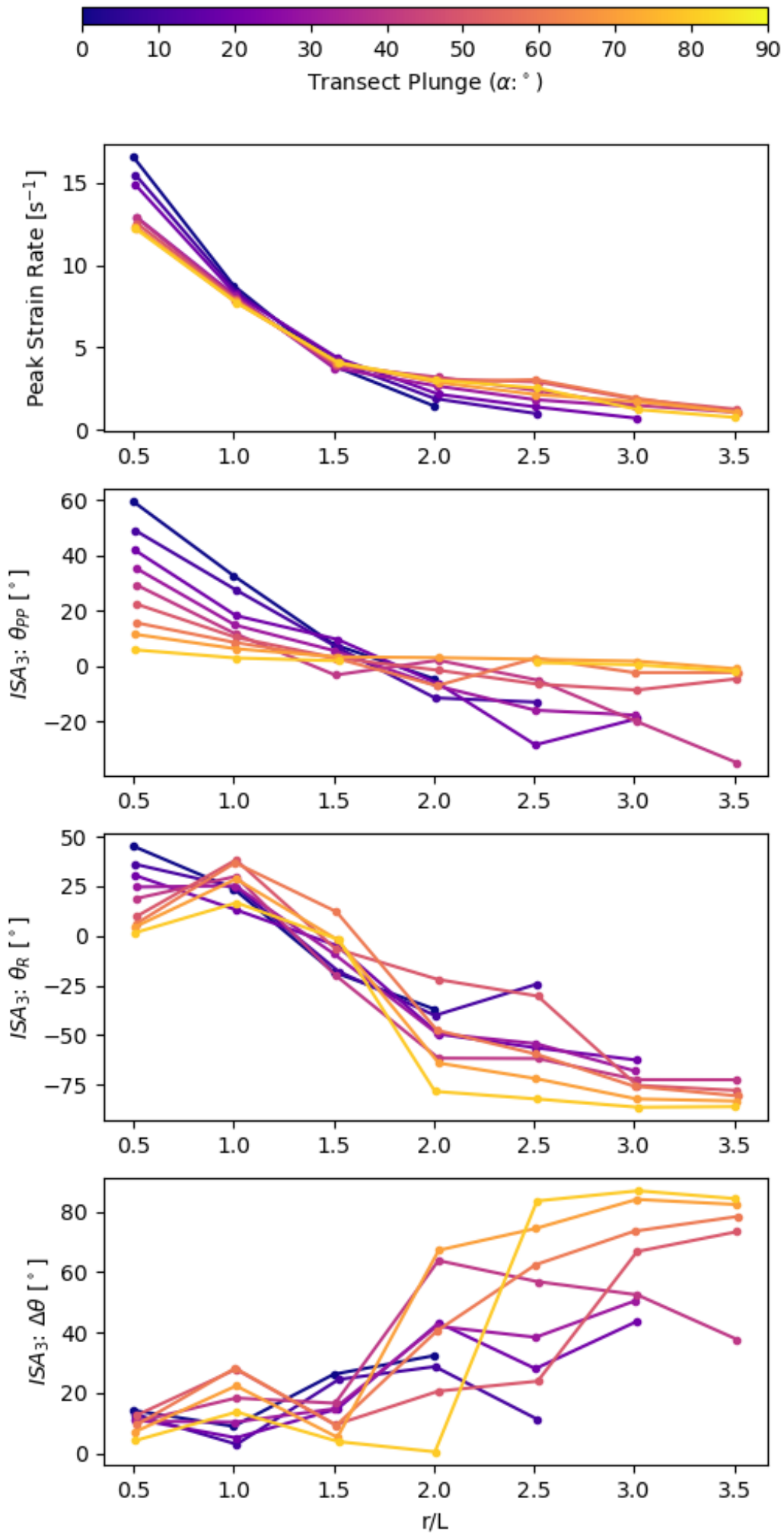

Figure 21. Instantaneous strain orientations for a $1 \mathrm{~km}$ diameter impactor travelling at 10 $\mathrm{km} / \mathrm{s}$. 
This is a post-print of an article published in ICARUS (doi: 10.1016/j.icarus.2021.114687).

$$
\underline{\mathrm{L}=1 \mathrm{~km}, \mathrm{vi}=20 \mathrm{~km} / \mathrm{s}}
$$

$-I S A_{3}$ at Peak Pressure $\quad-I S A_{3}$ at Release

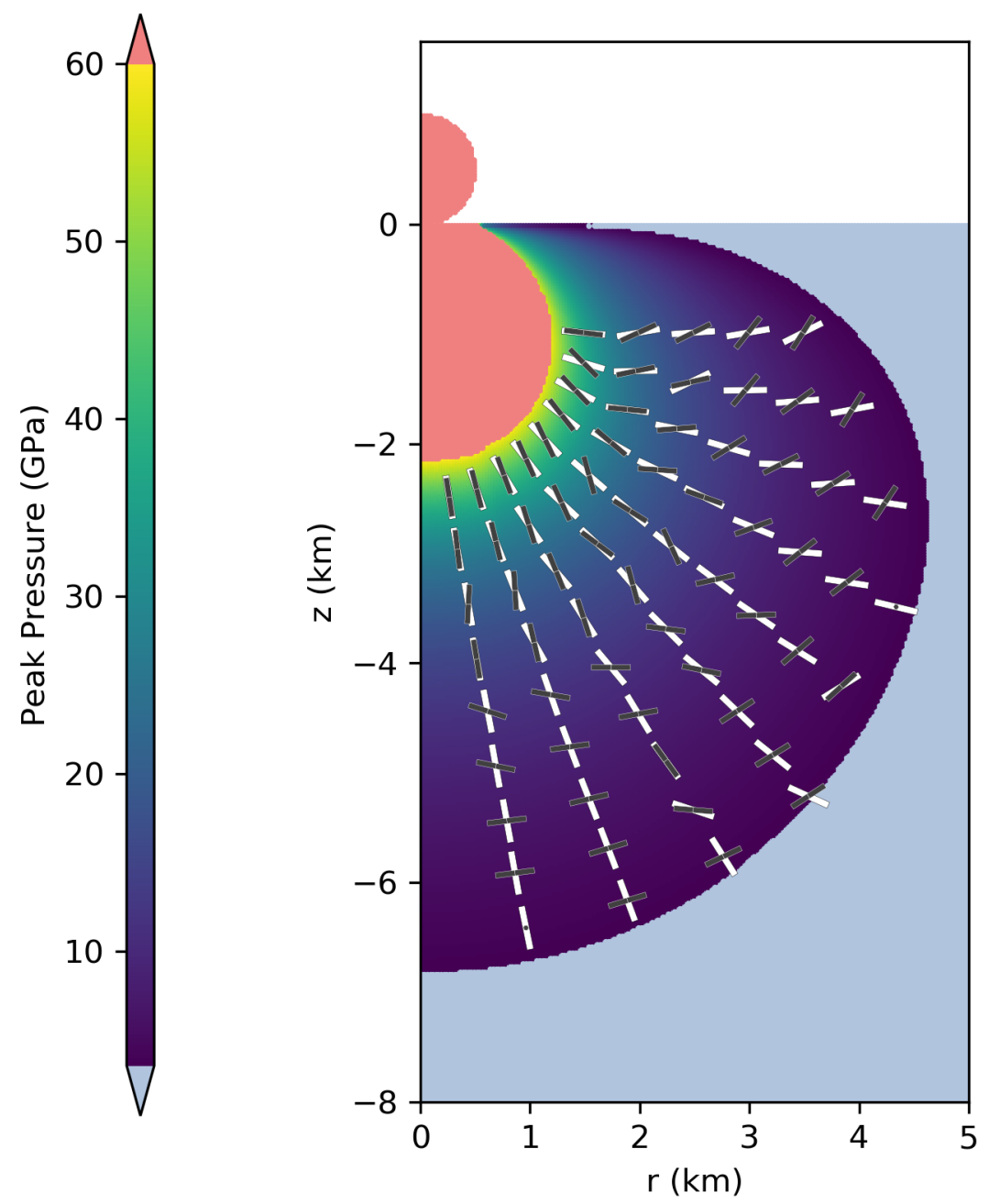

Figure 22. Instantaneous strain orientations for a $1 \mathrm{~km}$ diameter impactor travelling at 20 $\mathrm{km} / \mathrm{s}$. 
This is a post-print of an article published in ICARUS (doi: 10.1016/j.icarus.2021.114687).
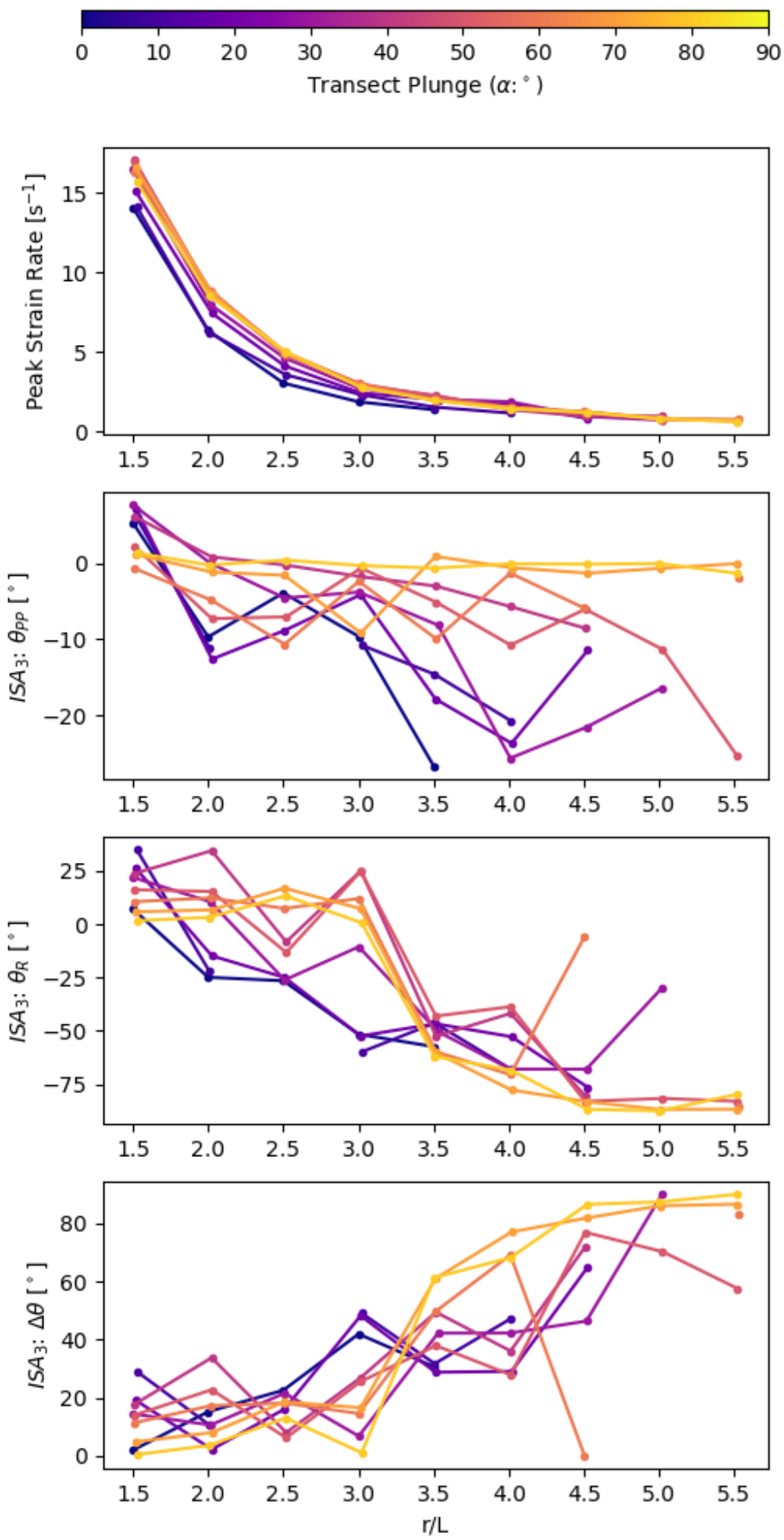

Figure 23. Instantaneous strain orientations for a $1 \mathrm{~km}$ diameter impactor travelling at 20 $\mathrm{km} / \mathrm{s}$. 
This is a post-print of an article published in ICARUS (doi: 10.1016/j.icarus.2021.114687).

Kinematic Vorticity

$$
\underline{\mathrm{L}=0.1 \mathrm{~km}, \mathrm{vi}=15 \mathrm{~km} / \mathrm{s}}
$$
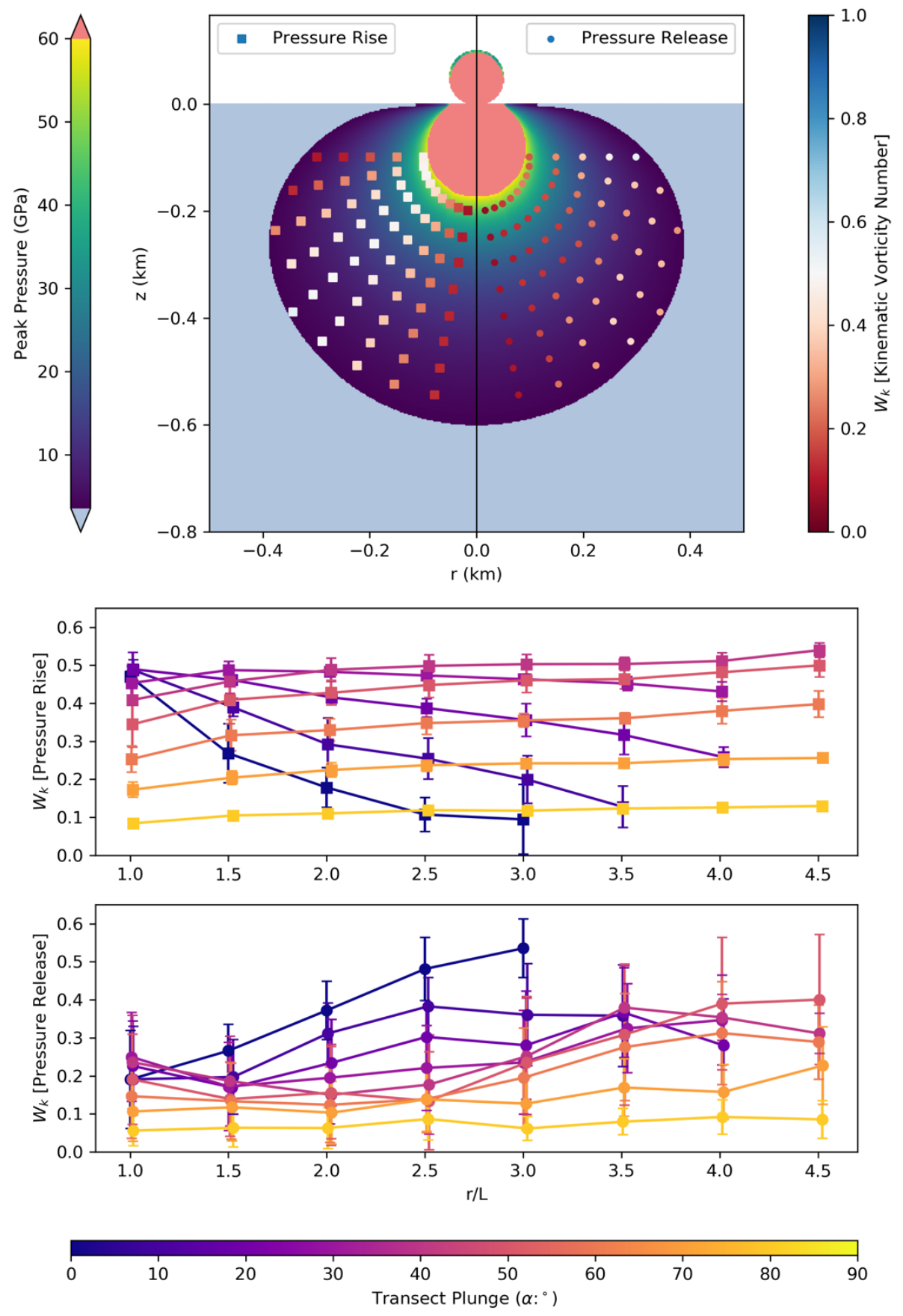

Figure 24. Kinematic vorticity number for a $0.1 \mathrm{~km}$ diameter impactor travelling at $15 \mathrm{~km} / \mathrm{s}$. 
This is a post-print of an article published in ICARUS (doi: 10.1016/j.icarus.2021.114687).

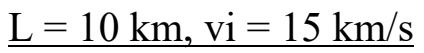
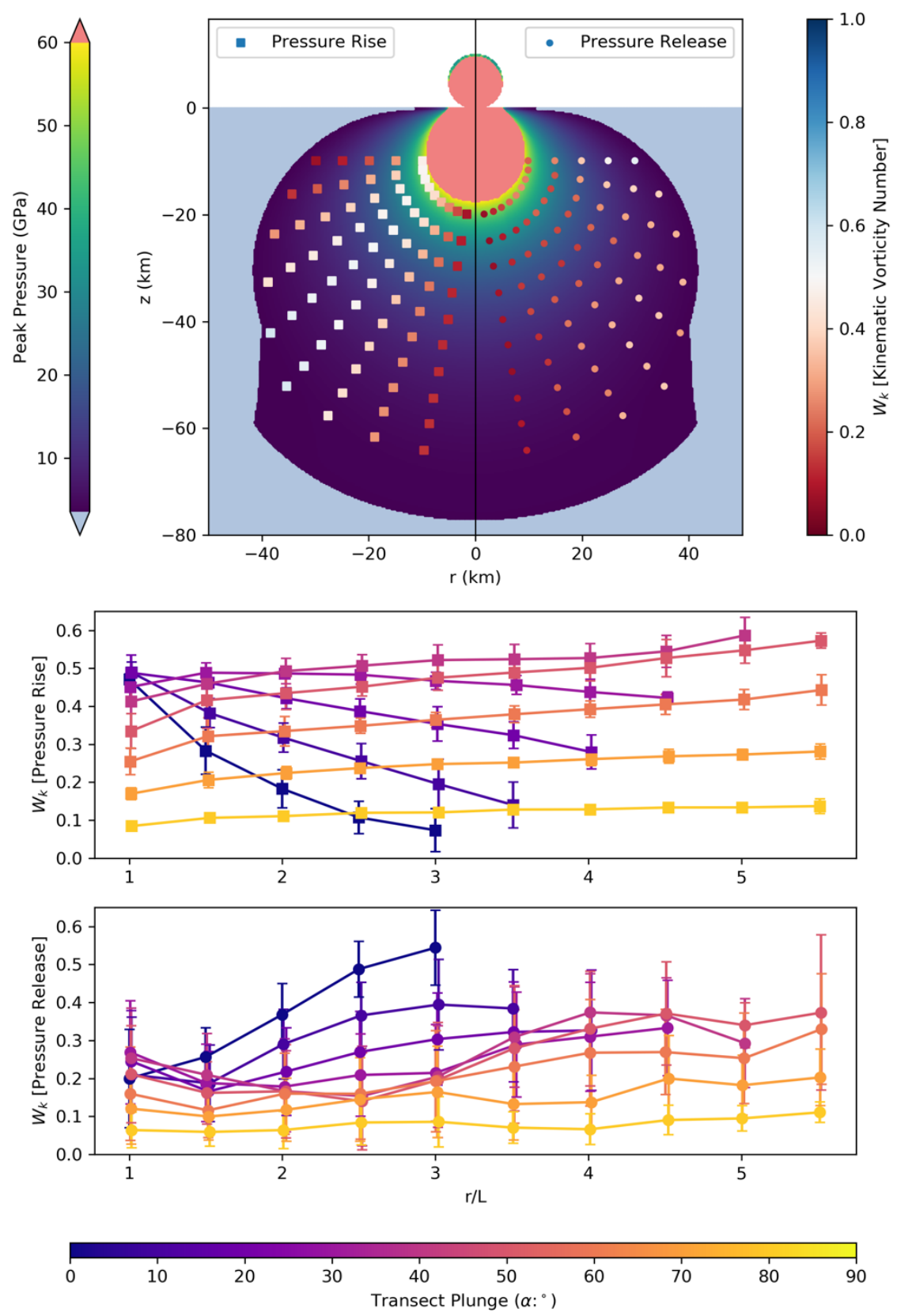

Figure 25. Kinematic vorticity number for a $10 \mathrm{~km}$ diameter impactor travelling at $15 \mathrm{~km} / \mathrm{s}$. 
This is a post-print of an article published in ICARUS (doi: 10.1016/j.icarus.2021.114687).

$$
\underline{\mathrm{L}}=1 \mathrm{~km}, \mathrm{vi}=10 \mathrm{~km} / \mathrm{s}
$$
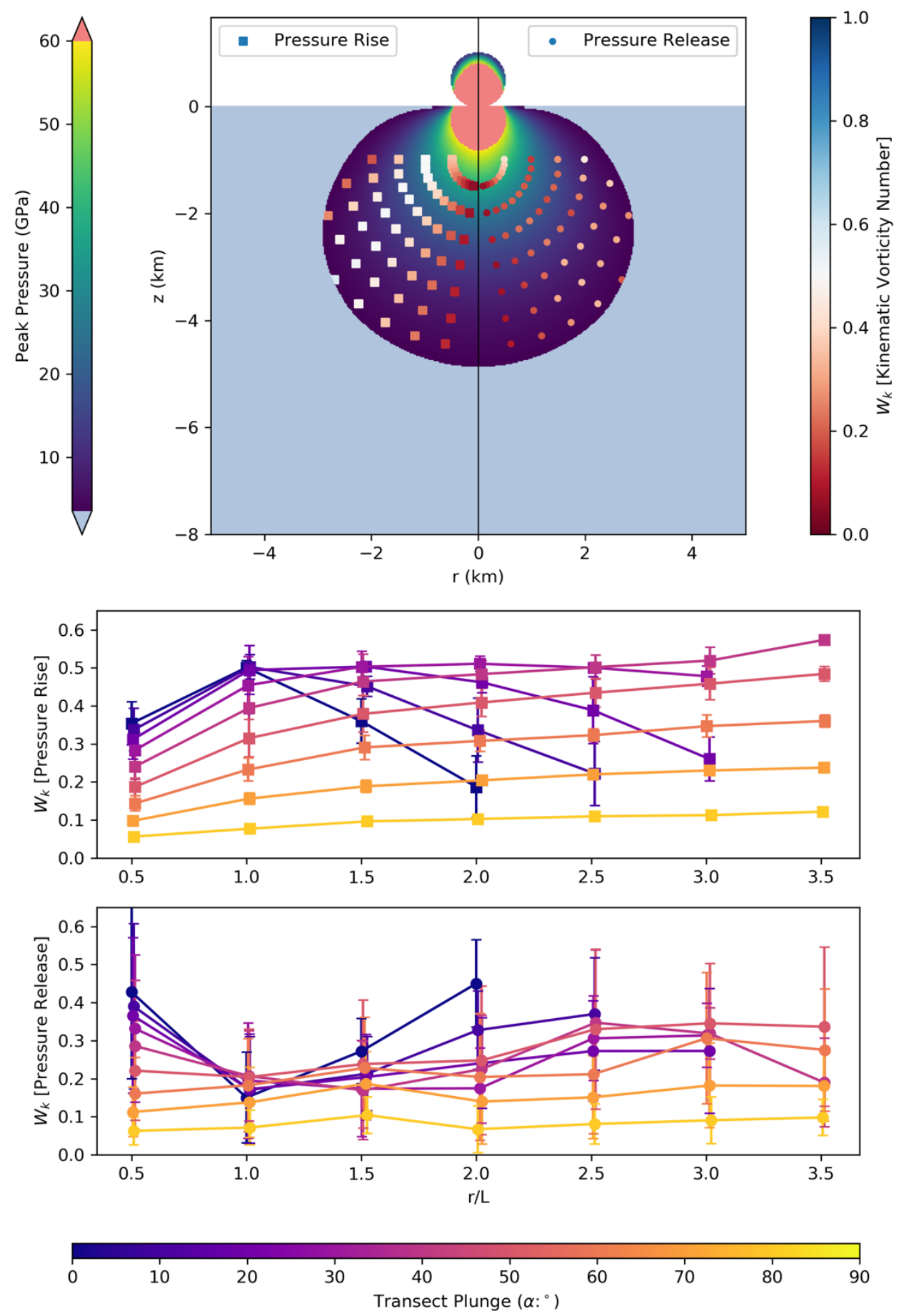

Figure 26. Kinematic vorticity number for a $1 \mathrm{~km}$ diameter impactor travelling at $10 \mathrm{~km} / \mathrm{s}$. 
This is a post-print of an article published in ICARUS (doi: 10.1016/j.icarus.2021.114687).

$$
\underline{\mathrm{L}}=1 \mathrm{~km}, \mathrm{vi}=20 \mathrm{~km} / \mathrm{s}
$$
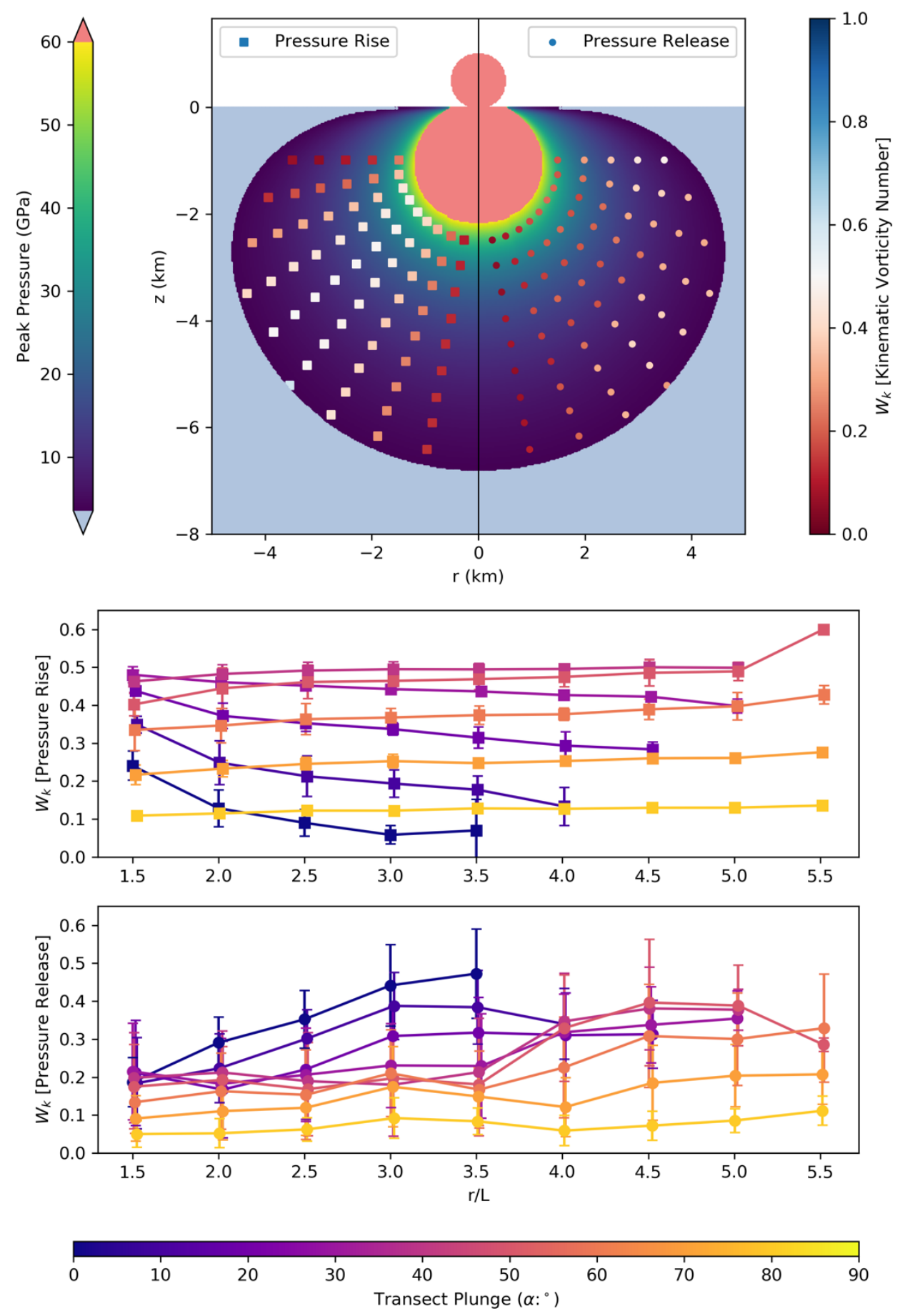

Figure 27. Kinematic vorticity number for a $1 \mathrm{~km}$ diameter impactor travelling at $20 \mathrm{~km} / \mathrm{s}$. 
This is a post-print of an article published in ICARUS (doi: 10.1016/j.icarus.2021.114687).

\section{Finite Strain Orientations}

$$
\underline{\mathrm{L}}=0.1 \mathrm{~km}, \mathrm{vi}=15 \mathrm{~km} / \mathrm{s}
$$

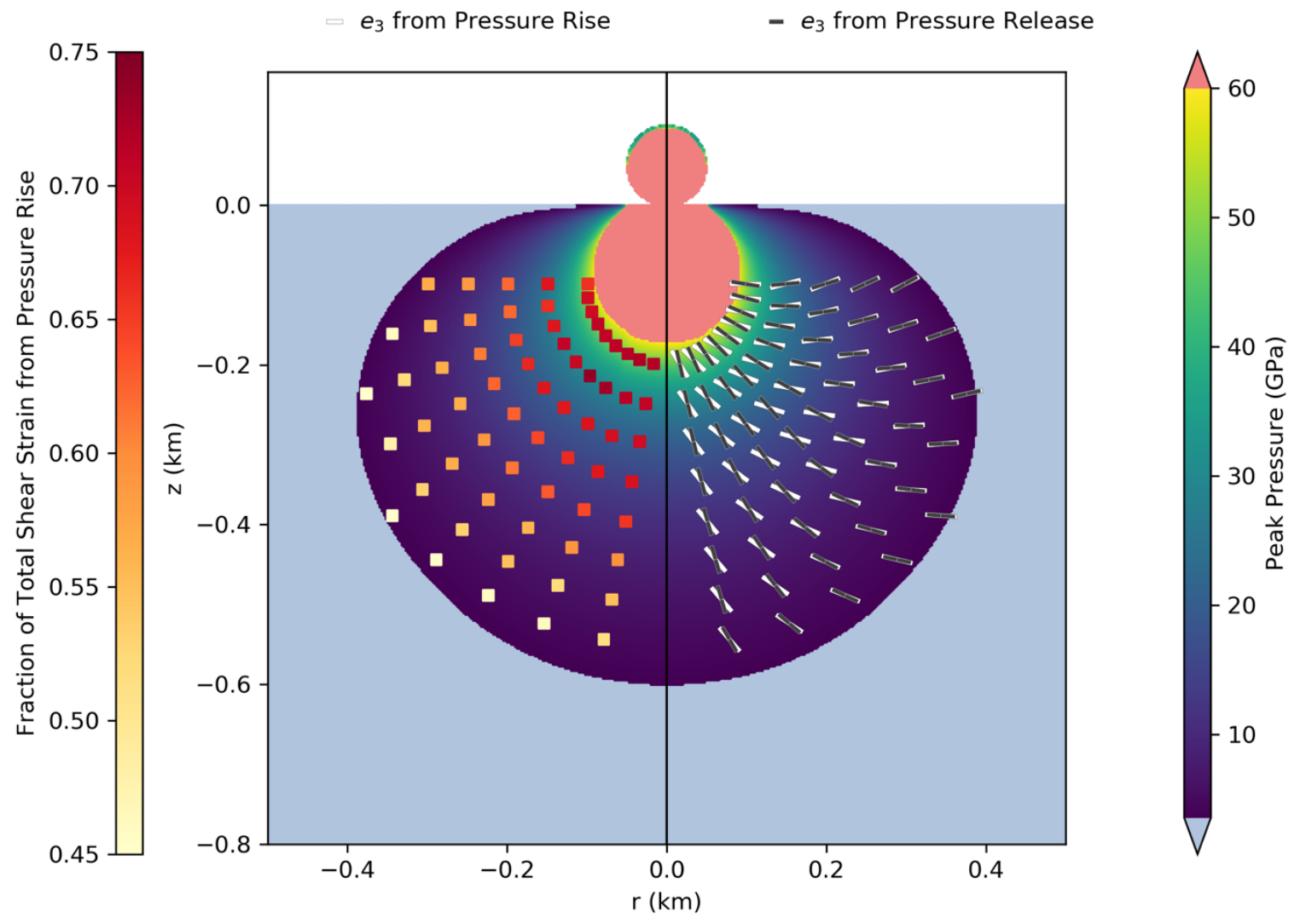

Figure 28. Finite strain orientations for a $0.1 \mathrm{~km}$ diameter impactor travelling at $15 \mathrm{~km} / \mathrm{s}$. 
This is a post-print of an article published in ICARUS (doi: 10.1016/j.icarus.2021.114687).
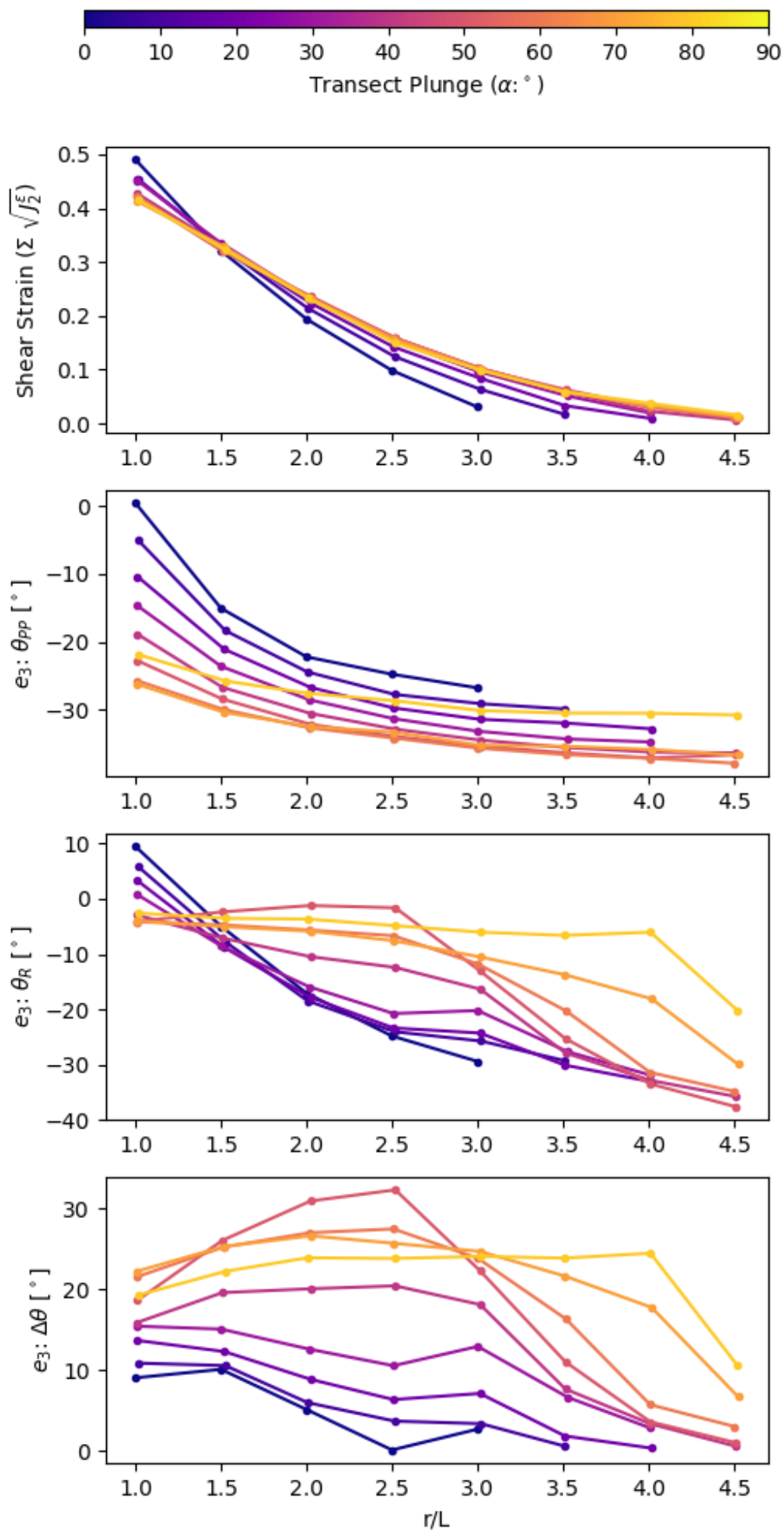

Figure 29. Finite strain orientations for a $0.1 \mathrm{~km}$ diameter impactor travelling at $15 \mathrm{~km} / \mathrm{s}$. 
This is a post-print of an article published in ICARUS (doi: 10.1016/j.icarus.2021.114687).

$$
\underline{\mathrm{L}}=10 \mathrm{~km}, \mathrm{vi}=15 \mathrm{~km} / \mathrm{s}
$$

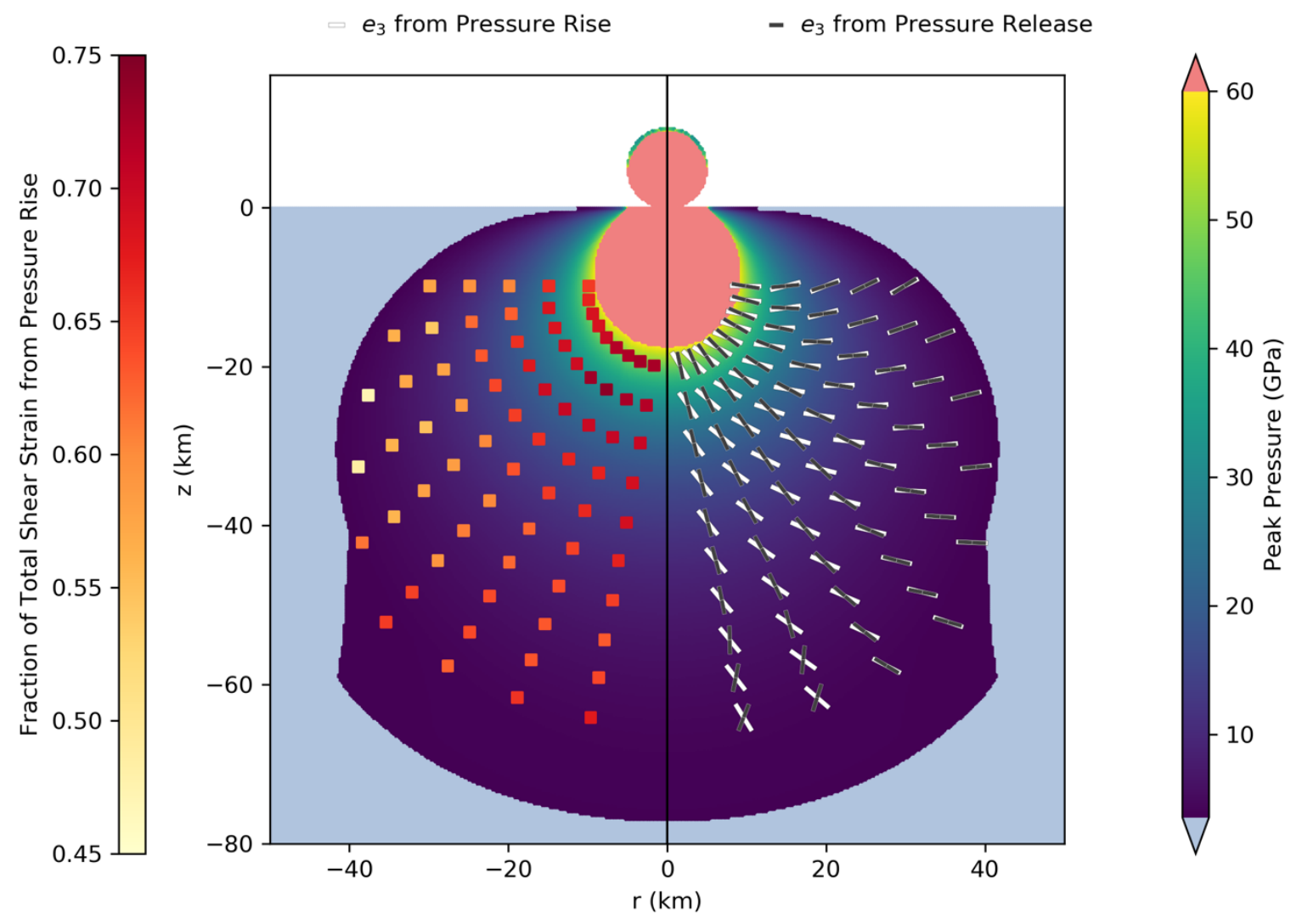

Figure 30. Finite strain orientations for a $10 \mathrm{~km}$ diameter impactor travelling at $15 \mathrm{~km} / \mathrm{s}$. 
This is a post-print of an article published in ICARUS (doi: 10.1016/j.icarus.2021.114687).
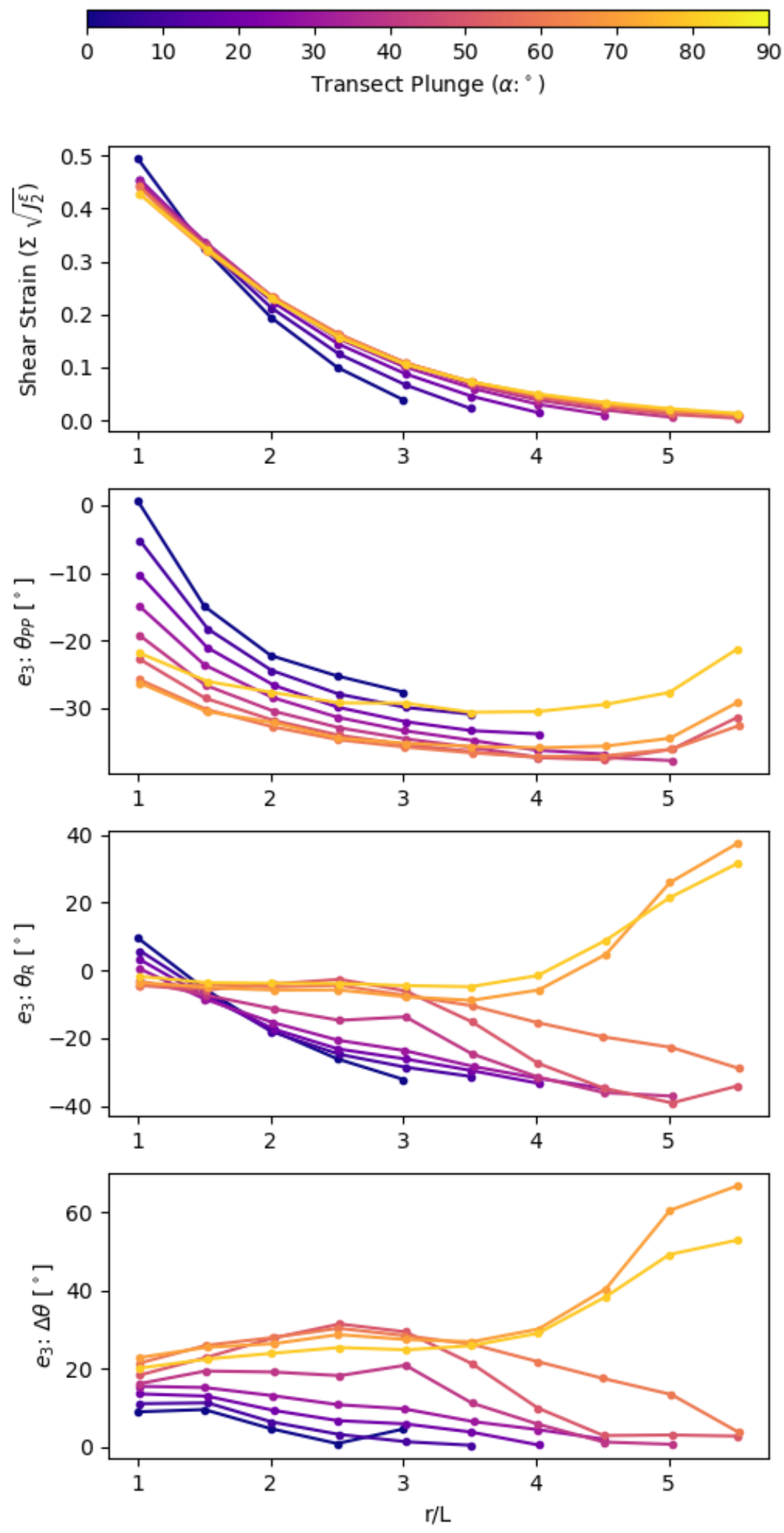

Figure 31. Finite strain orientations for a $10 \mathrm{~km}$ diameter impactor travelling at $15 \mathrm{~km} / \mathrm{s}$. 
This is a post-print of an article published in ICARUS (doi: 10.1016/j.icarus.2021.114687).

$$
\underline{\mathrm{L}=1 \mathrm{~km}, \mathrm{vi}=10 \mathrm{~km} / \mathrm{s}}
$$

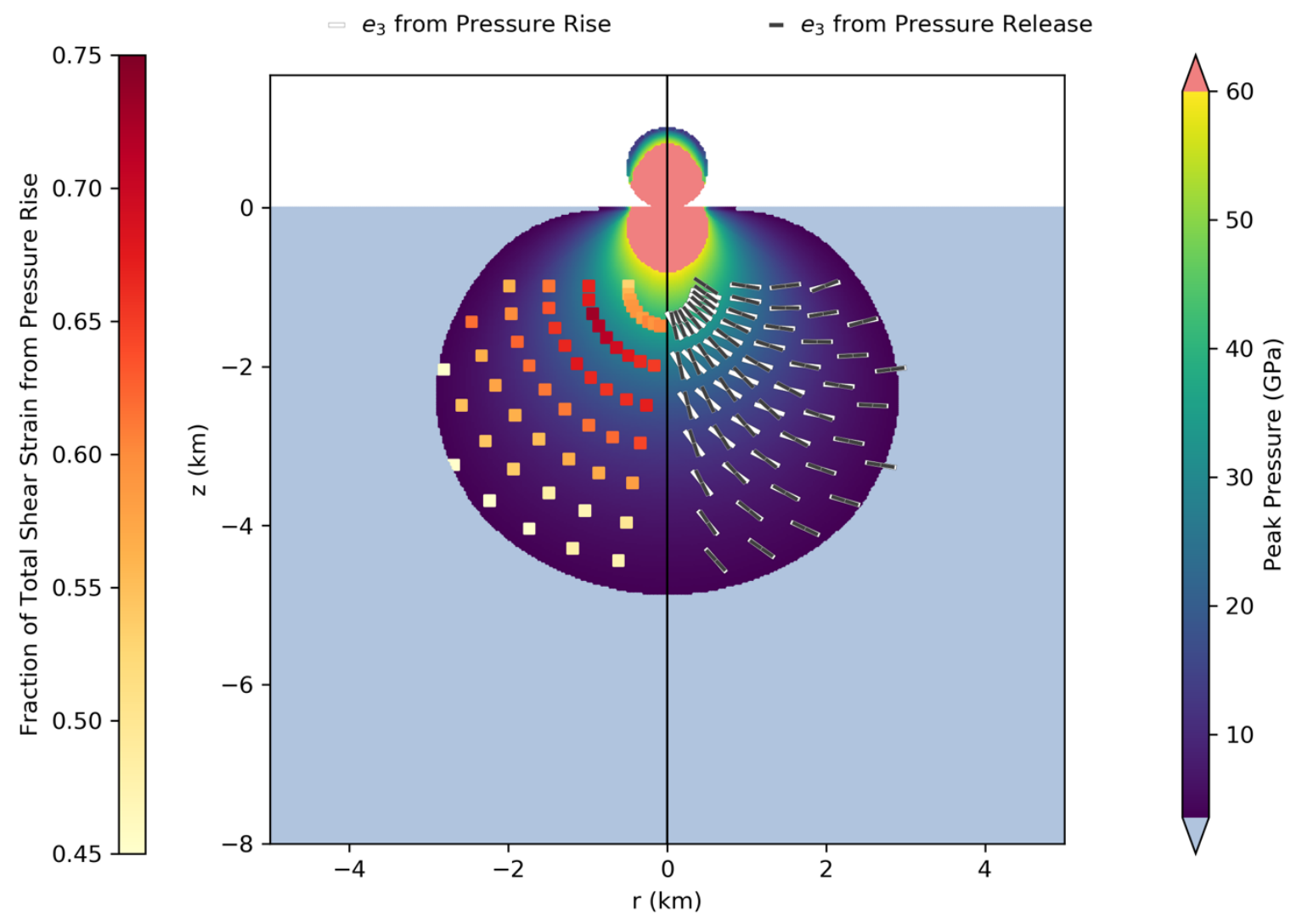

Figure 32. Finite strain orientations for a $1 \mathrm{~km}$ diameter impactor travelling at $10 \mathrm{~km} / \mathrm{s}$. 
This is a post-print of an article published in ICARUS (doi: 10.1016/j.icarus.2021.114687).
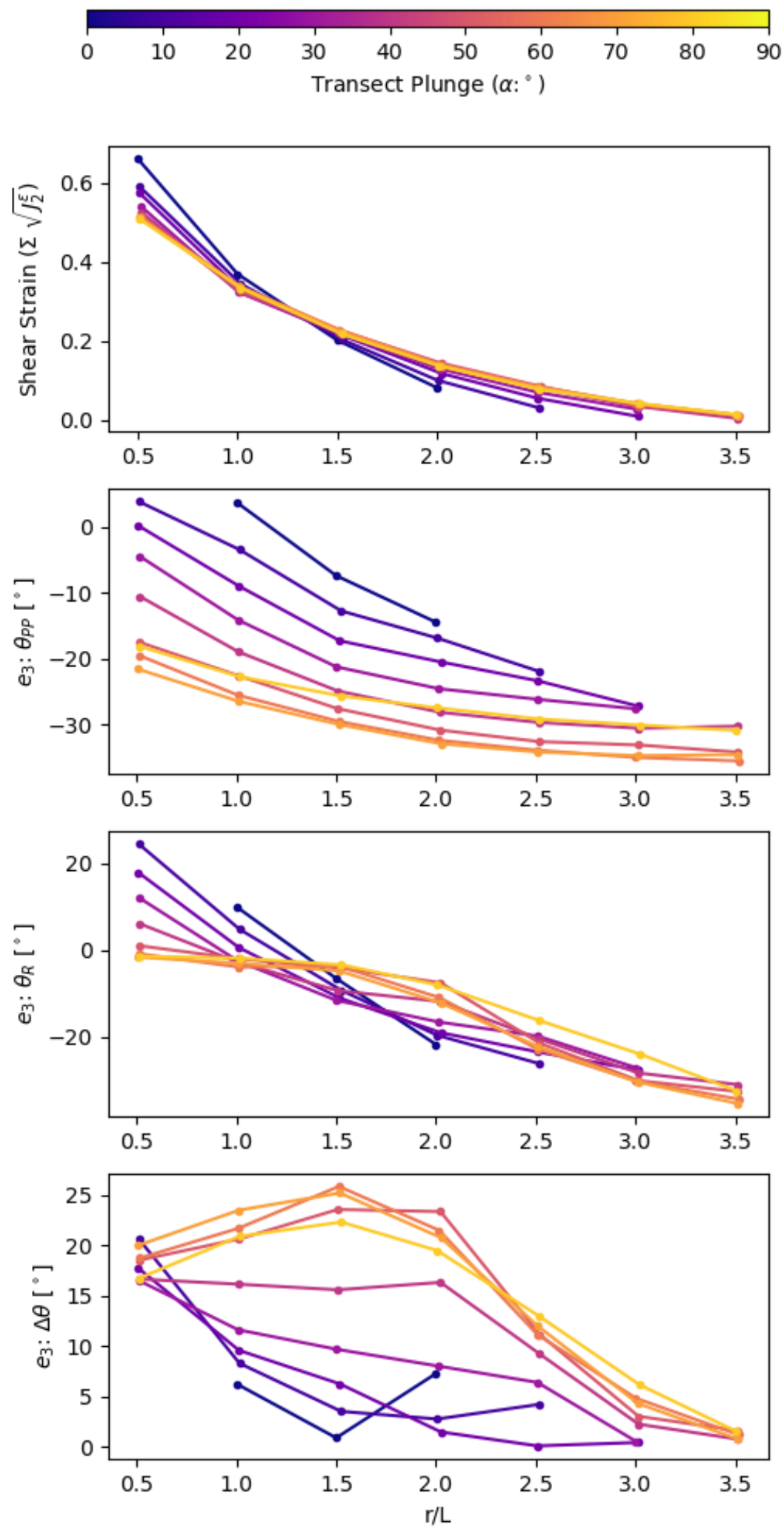

Figure 33. Finite strain orientations for a $1 \mathrm{~km}$ diameter impactor travelling at $10 \mathrm{~km} / \mathrm{s}$. 
This is a post-print of an article published in ICARUS (doi: 10.1016/j.icarus.2021.114687).

$$
\underline{\mathrm{L}=1 \mathrm{~km}, \mathrm{vi}=20 \mathrm{~km} / \mathrm{s}}
$$

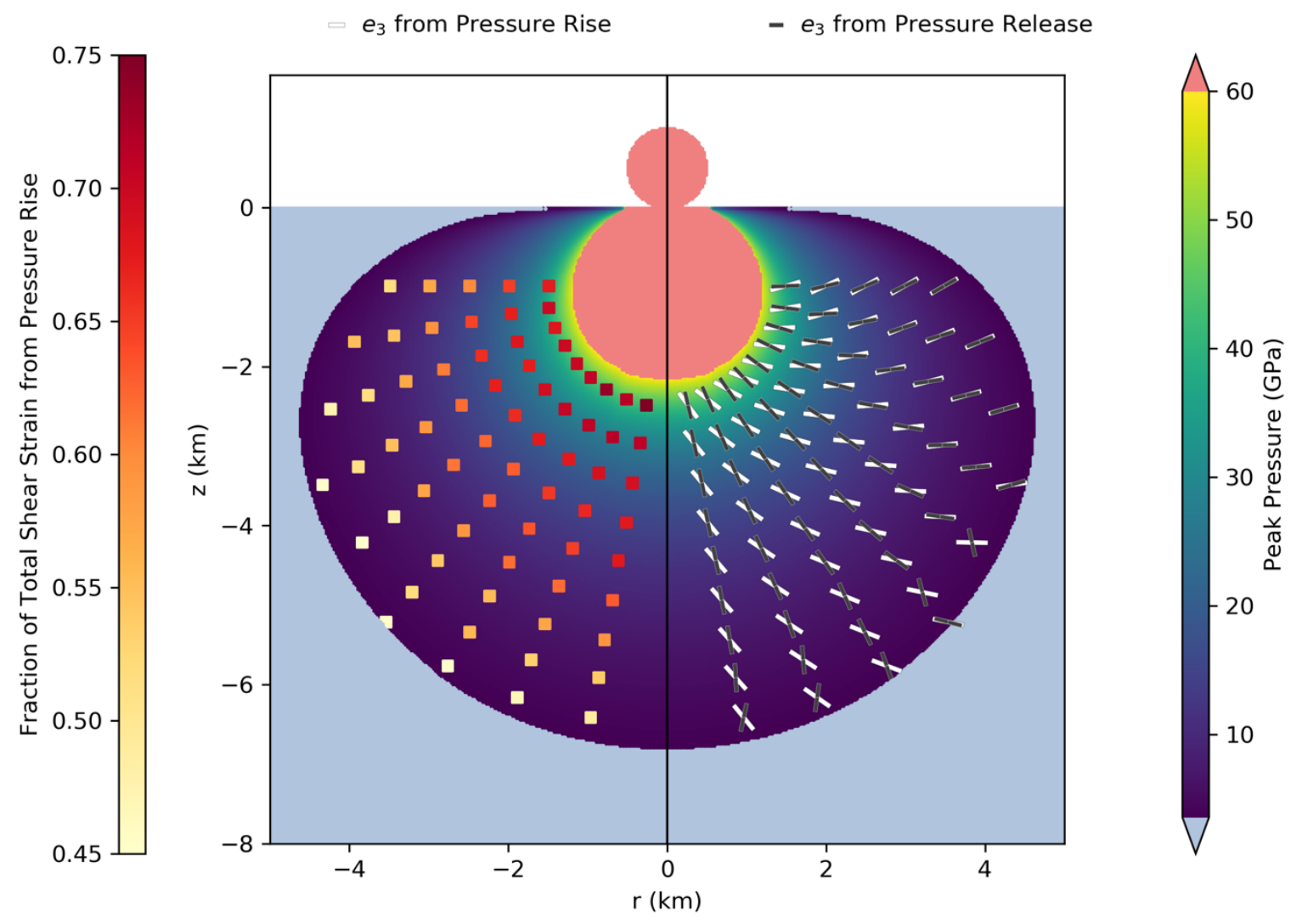

Figure 34. Finite strain orientations for a $1 \mathrm{~km}$ diameter impactor travelling at $20 \mathrm{~km} / \mathrm{s}$. 
This is a post-print of an article published in ICARUS (doi: 10.1016/j.icarus.2021.114687).
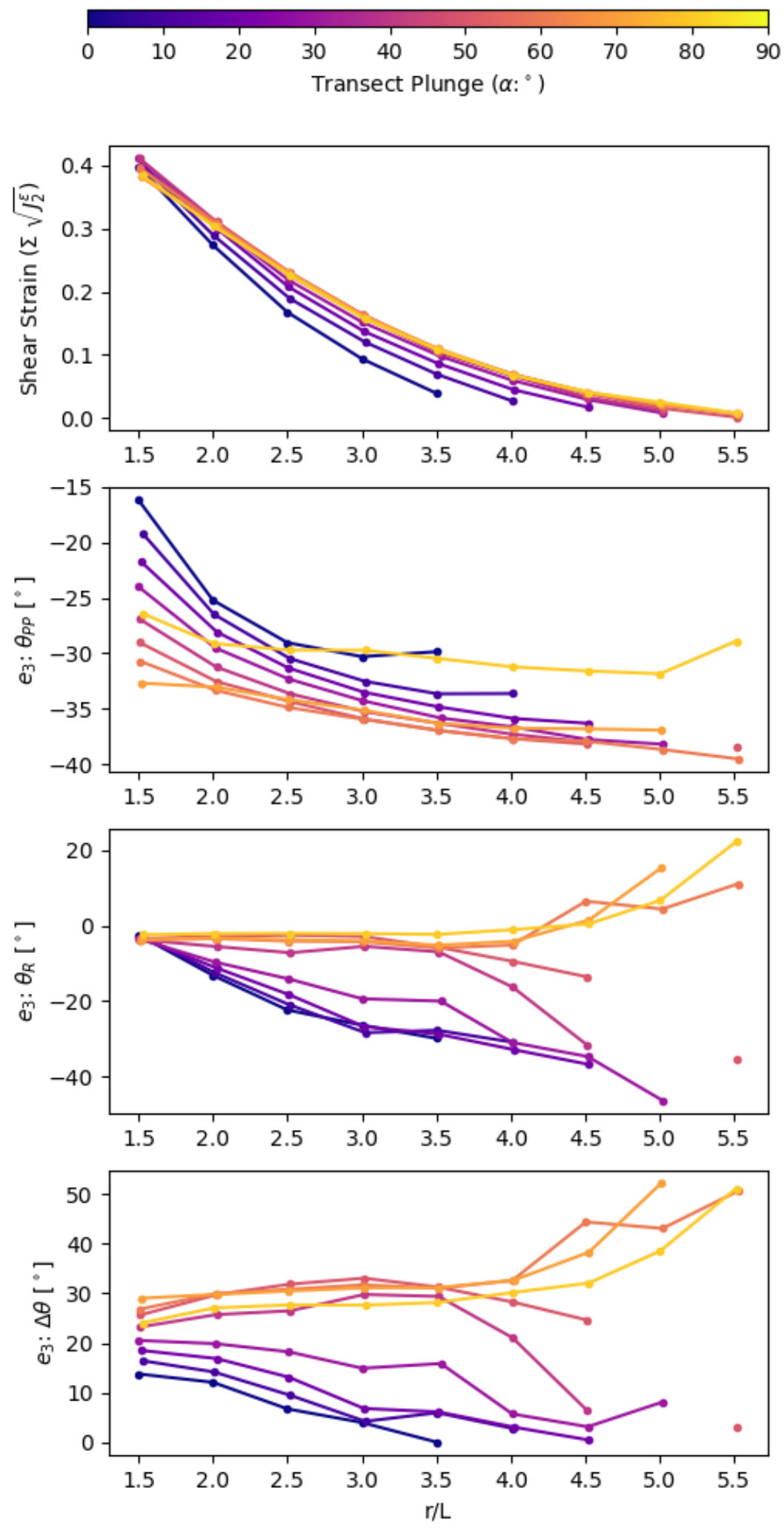

Figure 35. Finite strain orientations for a $1 \mathrm{~km}$ diameter impactor travelling at $20 \mathrm{~km} / \mathrm{s}$. 